\title{
Non-Cartan Mordell-Weil lattices of rational elliptic surfaces and heterotic/F-theory compactifications
}

\author{
Shun'ya Mizoguchi ${ }^{a}$ and Taro $\operatorname{Tani}^{b}$ \\ ${ }^{a}$ Theory Center, Institute of Particle and Nuclear Studies, KEK, \\ Tsukuba, Ibaraki 305-0801, Japan \\ ${ }^{b}$ National Institute of Technology, Kurume College, \\ Kurume, Fukuoka 830-8555, Japan \\ E-mail: mizoguchi@post.kek.jp, tani@kurume-nct.ac.jp
}

Abstract: The Mordell-Weil lattices (MW lattices) associated to rational elliptic surfaces are classified into 74 types. Among them, there are cases in which the MW lattice is none of the weight lattices of simple Lie algebras or direct sums thereof. We study how such "non-Cartan MW lattices" are realized in the six-dimensional heterotic/F-theory compactifications. In this paper, we focus on non-Cartan MW lattices that are torsion free and whose associated singularity lattices are sublattices of $A_{7}$. For the heterotic string compactification, a non-Cartan MW lattice yields an instanton gauge group $H$ with one or more $\mathrm{U}(1)$ group(s). We give a method for computing massless spectra via the index theorem and show that the U(1) instanton number is limited to be a multiple of some particular non-one integer. On the F-theory side, we examine whether we can construct the corresponding threefold geometries, i.e., rational elliptic surface fibrations over $\mathbb{P}^{1}$. Except for some cases, we obtain such geometries for specific distributions of instantons. All the spectrum derived from those geometries completely match with the heterotic results.

KeYwords: F-Theory, String Duality

ArXiv EPrint: 1808.08001 


\section{Contents}

1 Introduction 1

2 Models with non-Cartan type Mordell-Weil lattices 4

3 Heterotic index computations $\quad 6$

3.1 General method 6

3.2 Example 1: $G=\mathrm{SU}(7)\left(T=A_{6}\right)($ No.25) 7

3.3 Orthogonal decompositions of the $E_{8}$ root lattice: why a multiple of seven? 8

3.4 Example 2: $G=\mathrm{SU}(5) \times \mathrm{SU}(2)\left(T=A_{4} \oplus A_{1}\right)($ No.17) 9

3.4.1 The case when $H=\mathrm{SU}(2) \times \mathrm{U}(1) \times \mathrm{U}(1) \quad 9$

3.4.2 The case when $H=\mathrm{SU}(3) \times \mathrm{U}(1) \quad 11$

4 Geometries for non-Cartan Mordell-Weil lattices $\quad 13$

$\begin{array}{lll}\text { 4.1 } \mathrm{CY}_{3} \text { in six-dimensional F-theory/heterotic duality } & 13\end{array}$

$\begin{array}{lll}4.2 & \text { Mapping } \mathrm{CY}_{3} \text { to RES-fibered geometry } & 16\end{array}$

5 Geometry for $G=\mathrm{SU}(2) \times \mathrm{SU}(2) \times \mathrm{SU}(2)($ No.7) 20

$\begin{array}{lll}5.1 & \text { The heterotic spectrum } & 20\end{array}$

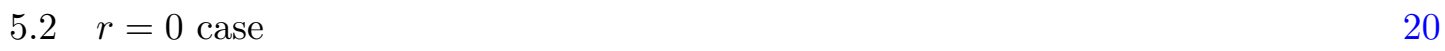

$\begin{array}{lll}5.3 & r \neq 0 \text { case } & 25\end{array}$

5.4 Looijenga's theorem 30

6 Geometry for non-Cartan SU(7) series $\quad 31$

$6.1 G=\mathrm{SU}(3) \times \mathrm{SU}(2) \times \mathrm{SU}(2)(\mathrm{No} .12)$

$6.2 G=\mathrm{SU}(5) \times \mathrm{SU}(2)(\mathrm{No} .17) \quad 33$

$6.3 G=\mathrm{SU}(3) \times \mathrm{SU}(4)(\mathrm{No} .19) \quad 34$

$6.4 G=\mathrm{SU}(7)($ No.25) 35

7 Geometry for non-Cartan SU(8) series $\quad 36$

$7.1 G=\mathrm{SU}(4) \times \mathrm{SU}(2) \times \mathrm{SU}(2)(\mathrm{No} .22) \quad 36$

$7.2 G=\mathrm{SU}(6) \times \mathrm{SU}(2)(\mathrm{No} .29) \quad 38$

$\begin{array}{lll}7.3 & \text { Other cases } & 39\end{array}$

$\begin{array}{lll}8 & \text { Conclusions } & 40\end{array}$

A Heterotic index computations for the SU(7) series $\quad 41$

A.1 $G=\mathrm{SU}(4) \times \mathrm{SU}(3)\left(T=A_{3} \oplus A_{2}\right)($ No.19) 41

A.1.1 The case when $H=\mathrm{SU}(2) \times \mathrm{U}(1) \times \mathrm{U}(1) \quad 41$

A.1.2 The case when $H=\mathrm{SU}(2) \times \mathrm{SU}(2) \times \mathrm{U}(1)$

A.2 $G=\mathrm{SU}(3) \times \mathrm{SU}(2) \times \mathrm{SU}(2)\left(T=A_{2} \oplus A_{1} \oplus A_{1}\right)(\mathrm{No} .12)$ 
B Heterotic index computations for the $\mathrm{SU}(8)$ series

B.1 $G=\mathrm{SU}(6) \times \mathrm{SU}(2)\left(T=A_{5} \oplus A_{1}\right)(\mathrm{No} .29) \quad 47$

B.2 $G=\mathrm{SU}(4) \times \mathrm{SU}(2) \times \mathrm{SU}(2)\left(T=A_{3} \oplus A_{1} \oplus A_{1}\right)(\mathrm{No} .22)$

C Charged matter spectrum from F-theory geometry $\quad 49$

$\begin{array}{lll}\text { C.1 } G=\mathrm{SU}(2) \times \mathrm{SU}(2) \times \mathrm{SU}(2)(r \neq 0) & 50\end{array}$

C.2 $G=\mathrm{SU}(3) \times \mathrm{SU}(2) \times \mathrm{SU}(2)(\mathrm{No} .12)$

C.3 $G=\mathrm{SU}(5) \times \mathrm{SU}(2)(\mathrm{No} .17) \quad 52$

C.4 $G=\mathrm{SU}(3) \times \mathrm{SU}(4)(\mathrm{No} .19) \quad 53$

C.5 $G=\mathrm{SU}(7)(\mathrm{No} .25) \quad 53$

C.6 $G=\mathrm{SU}(4) \times \mathrm{SU}(2) \times \mathrm{SU}(2)(\mathrm{No} .22) \quad 54$

C.7 $G=\mathrm{SU}(6) \times \mathrm{SU}(2)(\mathrm{No} .29)$

\section{Introduction}

F-theory [1-3] has a unique feature in modern particle physics model building based on string theory. The SU(5) GUT, which naturally explains the hypercharges of the observed quarks and leptons, and matter in the spinor representation of $\mathrm{SO}(10)$, into which all the quarks and leptons of a single generation are successfully incorporated - both are readily achieved in F-theory. F-theory models have an advantage over the $E_{8} \times E_{8}$ heterotic models as they may evade the issue of the GUT vs. Planck scales first addressed in [4]. F-theory can also generate Yukawa couplings that are perturbatively forbidden in D-brane models [5].

Almost a decade after the first formulation of F-theory, there was considerable development in understanding the local models [6-9] in terms of twisted super Yang-Mills theories or the Higgs bundles [10,11]. One of the notable findings in the development was the mechanism of the GUT gauge symmetry breaking by the fluxes turned on the brane. A large number of studies have been carried out in local models. An incomplete list includes [12-15].

Rather soon after this development, the Higgs particle was found at LHC in 2012, and the subsequent experiments showed that there was no low-energy supersymmetry. Later, the PLANCK data became also available to reveal that the energy of the inflation can be very high, even close to the GUT scale. These two new sources of knowledge have turned the focus of F-theory model building to global models. It is also known [3] that U(1) gauge symmetries in F-theory arise when the Mordell-Weil rank is nonzero, that is, when there are nontrivial global sections. This is in sharp contrast to nonabelian gauge symmetries, which can be solely determined by the singularity in the local model. Recent works on global models include [16-50].

For K3 surfaces, the Mordell-Weil rank varies depending on its Picard number. In contrast, the Mordell-Weil rank of a rational elliptic surface is always 10. Its Mordell-Weil group is known to be endowed with a lattice structure, and the possible pairs of the singularity and the corresponding Mordell-Weil lattice have been classified into 74 types [51]. 
Roughly speaking, the singularity lattice and the Mordell-Weil lattice are the orthogonal compliment of each other in the $E_{8}$ root lattice. In a typical case, the Mordell-Weil lattice coincides with a weight lattice of some semi-simple gauge group of the instantons in the dual heterotic string theory, and the singularity lattice is that of the unbroken gauge group. However, it is interesting to note that in the other cases the inner product matrix of the Mordell-Weil lattice is none of the (inverse of the) Cartan matrices of simple Lie algebras, nor is it their direct sum. It is these non-Cartan type Mordell-Weil lattices that we focus on in this paper. In fact, these are the cases where the gauge instanton includes some $\mathrm{U}(1)$ factor $(\mathrm{s}){ }^{1}$

We are particularly interested in the explicit forms of the Weierstrass equations of this special class of rational elliptic surfaces with a section ${ }^{2}$ which are fibered over $\mathbb{P}^{1}$ to form a complex threefold. This means that the parameters of the Weierstrass equations are sections of some line bundles over $\mathbb{P}^{1}[3] .^{3}$ Each of these geometries is regarded as a part of an elliptic K3 fibration in the stable degeneration limit [61-63] , and hence as a " $1 / 2$ CY threefold" since two such complex manifolds can be glued together into a K3-fibered Calabi-Yau threefold $\left(\mathrm{CY}_{3}\right)$.

In this paper, we will specifically consider a class of rational elliptic surfaces, and their fibrations over $\mathbb{P}^{1}$, which satisfy the following criteria:

1. The Mordel-Weil lattice $E(K)$ is neither a weight lattice of some semi-simple Lie algebra, nor is it a direct sum of such a weight lattice and a torsion.

2. The singularity lattice $T$ is a sublattice of $A_{7}$.

There are ten types of such rational elliptic surfaces in the Oguiso-Shioda classification, which are summarized in table 1 (See section 2).

To find an equation representing a "rational-elliptic-surface(RES)-fibered" complex threefold over $\mathbb{P}^{1}$ whose fiber rational elliptic surface belongs to this class, our strategy is to realize in the geometry the singularity $G$ of lattice $T$ associated to a given $E(K)$. First, we start from No.7 with singularity $G=\mathrm{SU}(2) \times \mathrm{SU}(2) \times \mathrm{SU}(2)$, which is not a nonCartan type, and construct the corresponding $\mathrm{CY}_{3}$ with $\mathrm{K} 3$ fibration. We then successively tune the complex structures ("unHiggsing") to achieve the necessary singularities $G$ of the respective types given in table 1 . Next, we map the obtained K3-fibered $\mathrm{CY}_{3}$ 's to RESfibered geometries. We carefully construct this map so that it does not change the structure

\footnotetext{
${ }^{1}$ As there are no such things as "U(1) instantons" in the ordinary four-dimensional noncompact Euclidean space, that might sound bizarre, but in a complex compact space they are nothing but line bundles with a nonzero first Chern class.

${ }^{2}$ Incidentally, the very same objects were studied in late 90's as Seiberg-Witten curves for E-strings [5258], though these rational elliptic surfaces were not supposed to be further fibered over anything then. A recent work on non-Cartan MW lattices for rational elliptic surfaces (and not their fibrations) is [59].

${ }^{3}$ Although we consider in this paper rational elliptic surfaces fibered over $\mathbb{P}^{1}$, the Weierstrass equations we obtain can be readily converted to those for a fibration over a complex two-fold $B_{2}$ by simply replacing a degree $a n+b$ polynomial in the affine coordinate $z^{\prime}$ of the $\mathbb{P}^{1}$, with a section of $\mathcal{L}^{-d} \otimes \mathcal{N}^{s}$ with $d=6 a-\frac{b}{2}$ and $s=a$, where $\mathcal{L}$ is the anti-canonical bundle of $B_{2}$ and $\mathcal{N}$ is the twisting line bundle determining the normal bundle of the fiber $\mathbb{P}^{1}$, which is the base of the rational elliptic surface. For further explanation of this correspondence, see [60].
} 
of the singularity. Through this strategy, we can successfully obtain the equations for all the $\mathrm{SU}(7)$ series (upper rows in table 1) and some (No.22 and No.29) of the $\mathrm{SU}(8)$ series (lower rows in table 1). We will also discuss why it is hard to find the equations for the remaining cases. We note that the equations for $\mathrm{SU}(8)$ series cannot be obtained by using Tate's form and we need to work in the Weierstrass form. This is because, if the coefficients $a_{i}(i=1,2,3,4,6)$ of Tate's form are assumed to have the necessary factors required from each singularity of the $\mathrm{SU}(8)$ series, then the total degrees of some $a_{i}$ (including $a_{3}$ and $a_{6}$ ) would exceed $i$, leading additional unwanted singularities.

We also compute the massless spectra of the dual heterotic string compactifications whose vector bundles are supposed to be determined by the RES-fibered spaces above $[2,3$, 64]. For all cases except No.45 in table 1, the instanton gauge group $H$ in the dual heterotic string theory has two or more irreducible group factors. In particular, we will see that $H$ specified by a non-Cartan Mordell-Weil lattice is typically a product of a semi-simple group and one or more $\mathrm{U}(1)$ group $(\mathrm{s})$. Thus one can distribute the total $12+n$ instanton numbers to each group factor. The $\mathrm{U}(1)$ instanton number(s) will be denoted by $r, \tilde{r}, \tilde{\tilde{r}}$, and so on. Although in principle there is no problem in applying the index theorem to these cases, the subtlety is that the multiplicities of some massless hypermultiplet then become fractional unless the $\mathrm{U}(1)$ instanton number is a multiple of some particular non-one integer. We will show why the $\mathrm{U}(1)$ instanton number cannot take an arbitrary integral value but must be a multiple by examining the orthogonal decomposition of the $E_{8}$ root lattice. This gives us a consistent integral number of hypermultiplets. For the cases of $\operatorname{rank}(E(K))>2$, more than one choices of the number of $\mathrm{U}(1)$ direction(s) are possible. In such cases, we obtain more than one massless spectra for a given $E(K)$ (and hence for a given $G$ ). We find that the spectrum is more general when the number of $\mathrm{U}(1)$ direction(s) is larger.

While it is possible to compute heterotic indices for the cases of distributed instantons, it is a nontrivial problem to obtain the equations for the RES-fibered spaces corresponding to such particularly distributed instantons. For every case of the non-Cartan type, where we have succeeded in finding an equation for the RES-fibered space, we show the complete match of the six-dimensional massless spectra read off from the Weierstrass equations on the F-theory side and those obtained by the index computations on the heterotic side for a special choice of instanton distribution. ${ }^{4}$

The outline of this paper is as follows: in section 2, we begin with an introduction to the non-Cartan type Mordell-Weil lattices. In section 3, we first review a general method to compute heterotic indices in six dimensions and demonstrate how it works in particular examples. We also discuss there why the instanton numbers must be a multiple of some particular non-one integer in general. In section 4 we review the basic facts on the sixdimensional F-theory/ heterotic duality, and explain our strategy to obtain the equation for a RES-fibered threefold having a non-Cartan Mordell-Weil lattice. In section 5, we start the construction by the No.7 case of the Oguiso-Shioda classification to obtain the equation for the case of a particular instanton distribution. We then deform this equation

\footnotetext{
${ }^{4}$ For the No.7 case, which is a Cartan type and a starting point of our construction, one can obtain the equation for an arbitrary distribution of instantons. The spectrum read off from the equation agrees with that of the dual heterotic theory. (In this case, the number of $\mathrm{SU}(2)$ instantons is parameterized by $r$.)
} 
in an appropriate way to find the equations for threefolds with arbitrarily distributed instantons. The match of the spectra is also verified there. Sections 6 and 7 are devoted to the considerations of the RES-fibered threefolds for the cases of the SU(7) series and the $\mathrm{SU}(8)$ series, respectively. They are constructed from the geometry of the No.7 case with a particular choice of instanton distributions. Finally we summarize our conclusions in section 8. In appendix A and B, we present the detail of the heterotic index computations for the $\mathrm{SU}(7)$ and the $\mathrm{SU}(8)$ series, respectively. Appendix $\mathrm{C}$ shows the explicit forms of the functions $f, g$ of the Weierstrass equations and the discriminant $\Delta$ for various cases considered in the text.

\section{Models with non-Cartan type Mordell-Weil lattices}

It is known that a rational elliptic surface $S$ possesses a lattice structure, called the MordellWeil lattice $E(K)$. ( $K$ is the field over which $S$ is defined.) When $S$ has no singularity, $E(K)$ is the self dual $E_{8}$ lattice. When $S$ has a singularity of an $A D E$ type with root lattice $T, E(K)$ is reduced, roughly speaking, to the orthogonal complement $T^{\perp}$ of $T$ in $E_{8}$. More precisely, $E(K)$ is the dual lattice of $T^{\perp}$ accompanied with a torsion part:

$$
E(K) \cong\left(T^{\perp}\right)^{*} \oplus \text { torsion, }
$$

where ${ }^{*}$ denotes the dual lattice. $T^{\perp}$ satisfies

$$
T \oplus T^{\perp} \subset \mathfrak{e}_{8} .
$$

In the context of the duality between F-theory and heterotic string, the root lattice $T$ of the $A D E$ singularity in $S$ corresponds to the gauge symmetry $G$, while the orthogonal complement $T^{\perp}$ corresponds to the gauge bundle $H$ of the heterotic string. The decomposition (2.2) is then interpreted as

$$
G \times H \subset E_{8}
$$

In other words (apart from the torsion part),

$$
\begin{aligned}
T & \leftrightarrow \text { gauge symmetry } G, \\
E(K) & \leftrightarrow \text { dual gauge bundle } H^{*} .
\end{aligned}
$$

The Mordell-Weil lattices $E(K)$ are classified into 74 patterns [51]. In many cases, $\left(T^{\perp}\right)^{*}$ is a weight lattice of some $A D E$ type Lie algebra or a direct sum thereof, but there are some special cases where $\left(T^{\perp}\right)^{*}$ is not a weight lattice of any $A D E$ type Lie algebra. In these cases $T^{\perp}$ is also not a root lattice of any $A D E$ type, and neither is the gauge bundle $H$ of the heterotic string. Let us call these Mordell-Weil lattices $E(K)$ and the corresponding gauge bundles $H$ "non-Cartan type". In this paper, among them, we will study the cases listed in table 1 , where $T$ is a subalgebra of $A_{7}$ and $E(K)$ is torsion free.

In the table, $\Lambda_{(N)}$ is a matrix representing the lattice $E(K) \cong\left(T^{\perp}\right)^{*}$. The inverse $\Lambda_{(N)}^{-1}$ representing the dual lattice $T^{\perp}$, or equivalently, the gauge bundle $H$, takes one of 


\begin{tabular}{|c|c|c|}
\hline No. & $T \leftrightarrow G$ & $E(K) \leftrightarrow H^{*}$ \\
\hline 25 & $A_{6}$ & $\Lambda_{(25)}$ \\
17 & $A_{4} \oplus A_{1}$ & $\Lambda_{(17)}$ \\
19 & $A_{3} \oplus A_{2}$ & $\Lambda_{(19)}$ \\
12 & $A_{2} \oplus A_{1} \oplus A_{1}$ & $\Lambda_{(12)}$ \\
\hline 45 & $A_{7}$ & $\langle 1 / 8\rangle$ \\
29 & $A_{5} \oplus A_{1}$ & $A_{1}^{*} \oplus\langle 1 / 6\rangle$ \\
31 & $A_{4} \oplus A_{2}$ & $\Lambda_{(31)}$ \\
36 & $A_{3} \oplus A_{3}$ & $\langle 1 / 4\rangle \oplus\langle 1 / 4\rangle$ \\
20 & $A_{2} \oplus A_{2} \oplus A_{1}$ & $A_{2}^{*} \oplus\langle 1 / 6\rangle$ \\
22 & $A_{3} \oplus A_{1} \oplus A_{1}$ & $A_{1}^{*} \oplus A_{1}^{*} \oplus\langle 1 / 4\rangle$ \\
\hline
\end{tabular}

Table 1. The non-Cartan type Mordell-Weil lattices $E(K)$ that are torsion free and associated $T$ are sublattices of $A_{7}$ [51].

the following non-Cartan forms:

$$
\begin{aligned}
\Lambda_{(25)}^{-1} & =\left(\begin{array}{cc}
4 & -1 \\
-1 & 2
\end{array}\right), & \Lambda_{(17)}^{-1} & =\left(\begin{array}{ccc}
4 & -1 & 1 \\
-1 & 2 & -1 \\
1 & -1 & 2
\end{array}\right), \quad \Lambda_{(19)}^{-1}=\left(\begin{array}{ccc}
2 & 0 & -1 \\
0 & 2 & -1 \\
-1 & -1 & 4
\end{array}\right), \\
\Lambda_{(12)}^{-1} & =\left(\begin{array}{cccc}
4 & -1 & 0 & 1 \\
-1 & 2 & -1 & 0 \\
0 & -1 & 2 & -1 \\
1 & 0 & -1 & 2
\end{array}\right), & \Lambda_{(31)}^{-1} & =\left(\begin{array}{cc}
8 & -1 \\
-1 & 2
\end{array}\right) .
\end{aligned}
$$

$A_{n}^{*}, D_{n}^{*}$ are the weight lattices of $A_{n}, D_{n} .\langle 1 / k\rangle$ denotes the one-dimensional lattice with lattice spacing $\sqrt{\frac{1}{k}}$. Its dual lattice has a non-Cartan form unless $k=2$, in which the lattice becomes the $A_{1}$ weight lattice.

In terms of heterotic string, the two series in table 1 are obtained by Higgsing the gauge group $G=\mathrm{SU}(7)$ (No.25) and $G=\mathrm{SU}(8)$ (No.45), respectively. Their Higgsing chains are summarized as follows: ${ }^{5}$ (the subscripts are the numbers in table 1 )

$$
\begin{aligned}
& \mathrm{SU}(5) \times \mathrm{SU}(2)_{(17)} \\
& \stackrel{\nearrow}{\mathrm{SU}(7)_{(25)} \rightarrow \mathrm{SU}(3) \times \mathrm{SU}(4)_{(19)}} \stackrel{\searrow}{\rightarrow} \mathrm{SU}(3) \times \mathrm{SU}(2) \times \mathrm{SU}(2)_{(12)} \\
& \nearrow_{\nearrow}^{\mathrm{SU}(4) \times \mathrm{SU}(4)_{(36)} \rightarrow \mathrm{SU}(4) \times \mathrm{SU}(2) \times \mathrm{SU}(2)_{(22)}} \\
& \mathrm{SU}(8)_{(45)} \rightarrow \mathrm{SU}(6) \times \mathrm{SU}(2)_{(29)} \\
& \searrow_{\mathrm{SU}(3) \times \mathrm{SU}(5)_{(31)} \stackrel{\searrow}{\rightarrow} \mathrm{SU}(3) \times \mathrm{SU}(3) \times \mathrm{SU}(2)_{(20)}}
\end{aligned}
$$

\footnotetext{
${ }^{5} G=\mathrm{SU}(2) \times \mathrm{SU}(2) \times \mathrm{SU}(2) \times \mathrm{SU}(2)(\mathrm{No} .14$ of [51]) belongs to the Higgsing chain of $G=\mathrm{SU}(8)$, but we excluded it from table 1 because it has $E(K)=A_{1} \oplus A_{1} \oplus A_{1} \oplus A_{1}$, which is not the non-Cartan type.
} 


\section{$3 \quad$ Heterotic index computations}

\subsection{General method}

We will first quickly review the general method to compute the heterotic spectrum in six dimensions.

The Dirac index for a six-dimensional compactification of heterotic string theory on a complex 2-fold is given by

$$
\text { index }=\left.\int \hat{A}(T Z) \operatorname{ch}(V)\right|_{4},
$$

where $\hat{A}(T Z)$ is the A-roof genus of the tangent bundle of the complex 2-fold $Z$ on which the heterotic string is compactified, and $\operatorname{ch}(V)$ is the Chern character of the vector bundle $V$ over $Z$. The number of hypermultiplets is given by $-1 / 2$ of the index, where the overall minus sign is a convention and the factor of $1 / 2$ is due to the fact that the heterotic gaugino is a Majorana-Weyl spinor in ten dimensions.

We consider the cases where the instanton takes values in a subgroup $H$ of, say, the first factor of $E_{8}$ to leave the centralizer subgroup $G$ of $H$ in $E_{8}$ unbroken. We are particularly interested in the cases where $H$ contains some U(1) factors. As in [65], let the $\mathbf{2 4 8}$ of $E_{8}$ be decomposed into the representations of $G \times H$ as

$$
\mathbf{2 4 8}=\oplus_{i} L_{i} \otimes C_{i},
$$

where $L_{i}$ and $C_{i}$ are irreducible representations of $G$ and $H$, respectively. Using the fact that $\int_{\mathrm{K} 3} p_{1}=-48$, the number of hypermultiplet $n_{L_{i}, C_{i}}$ in a representation $L_{i}$ of $G$ (and $C_{i}$ of $\left.H\right)$ is given by [65]

$$
\begin{aligned}
n_{L_{i}, C_{i}} & \equiv-\frac{1}{2} \text { index } \\
& =-\operatorname{dim} C_{i}+\frac{1}{2} \cdot \frac{1}{8 \pi^{2}} \int_{\mathrm{K} 3} \operatorname{Tr}_{C_{i}} F \wedge F,
\end{aligned}
$$

where $F$ is the instanton gauge field 2-form taking values in (the Lie algebra of) $H$. The trace is taken in the representation $C_{i}$.

The equation (3.3) is still a correct formula even for the cases where $H$ contains some $\mathrm{U}(1)$ factors. Since

$$
\begin{aligned}
\sum_{a=1,2} \sum_{i} \operatorname{dim} L_{i}^{(a)} \cdot \frac{1}{8 \pi^{2}} \int \operatorname{Tr}_{C_{i}^{(a)}} F^{(a)} \wedge F^{(a)} & =\frac{1}{8 \pi^{2}} \int_{\mathrm{K} 3} \operatorname{Tr}_{\mathbf{2 4 8} \oplus \mathbf{2 4 8}} F \wedge F \\
& =-30 \int_{\mathrm{K} 3} p_{1} \\
& =30 \cdot 48
\end{aligned}
$$

due to the anomaly cancellation condition, where $a=1,2$ are the labels to distinguish the $E_{8}$ factors, we see that the second term of (3.3) is nothing but -30 times the contribution of each representation $C_{i}$ to the total instanton number $(=24$, taking the factor of $1 / 2$ into account). The normalizations of the traces and $U(1)$ charges are determined once the instanton number of each irreducible group factor of $H$ is specified. 


\subsection{Example 1: $G=\mathrm{SU}(7)\left(T=A_{6}\right)(\mathrm{No.25})$}

In this case one can take $\mathrm{SU}(2) \times \mathrm{U}(1)$ as $H$. Let the instanton numbers of $\mathrm{SU}(2)$ and $\mathrm{U}(1)$ be $12+n-r$ and $r$, respectively, so that the total instanton number of $H$ is $12+n$. Then we have ${ }^{6}$

$$
\frac{1}{8 \pi^{2}} \int F^{A} \wedge F^{B} \operatorname{Tr}_{\mathbf{2 4 8}} \operatorname{ad} T^{A} \operatorname{ad} T^{B}=60(12+n) .
$$

The adjoint representation of $E_{8}$ is decomposed as

$$
\begin{aligned}
& E_{8} \supset \mathrm{SU}(7) \times \mathrm{SU}(2) \times \mathrm{U}(1), \\
& \mathbf{2 4 8}=(\mathbf{4 8}, \mathbf{1})_{0} \oplus(\mathbf{1}, \mathbf{1})_{0} \oplus(\mathbf{7}, \mathbf{1})_{-4} \oplus(\overline{\mathbf{7}}, \mathbf{1})_{4} \\
& \oplus(\mathbf{3 5}, \mathbf{1})_{2} \oplus(\overline{\mathbf{3 5}}, \mathbf{1})_{-2} \oplus(\mathbf{1}, \mathbf{3})_{0} \\
& \oplus(\mathbf{7}, \mathbf{2})_{3} \oplus(\overline{\mathbf{7}}, \mathbf{2})_{-3} \oplus(\mathbf{2 1}, \mathbf{2})_{-1} \oplus(\overline{\mathbf{2 1}}, \mathbf{2})_{1},
\end{aligned}
$$

where the subscripts denote the $\mathrm{U}(1)$ charges. This yields

$$
\begin{aligned}
\frac{1}{8 \pi^{2}} \int F^{A} \wedge F^{B} \operatorname{Tr}_{248} \operatorname{ad} T^{A} \operatorname{ad} T^{B}= & \frac{1}{8 \pi^{2}} \int F^{a} \wedge F^{b} \cdot 60 \operatorname{Tr} \tau^{a} \tau^{b} \\
& +\frac{1}{8 \pi^{2}} \int F^{\mathrm{U}(1)} \wedge F^{\mathrm{U}(1)} \cdot 14 \cdot 60,
\end{aligned}
$$

where $\tau^{a}$ are the $\mathbf{2}$ representation matrices of $\mathrm{SU}(2)$, and $14 \cdot 60$ is the sum of the $\mathrm{U}(1)$ charge squares. As we assumed above, the first term is equal to $60(12+n-r)$, whereas the second term is $60 \mathrm{r}$. Therefore we have

$$
\begin{aligned}
\frac{1}{8 \pi^{2}} \int F^{a} \wedge F^{b} \operatorname{Tr} \tau^{a} \tau^{b} & =12+n-r, \\
\frac{1}{8 \pi^{2}} \int F^{\mathrm{U}(1)} \wedge F^{\mathrm{U}(1)} & =\frac{r}{14},
\end{aligned}
$$

which means that (Note the factor of $-1 / 2$ in eq. (3.3)) a 2 representation of $\mathrm{SU}(2)$ contributes $\frac{12+n-r}{2}$, whereas each $\mathrm{U}(1)$-charge $Q$ component contributes $\frac{Q^{2}}{2} \cdot \frac{r}{14}$, to the multiplicities of hypermultiplets transforming in the corresponding $G$ representation.

As an illustration let us compute $n_{\mathbf{2 1 , 2} \mathbf{2}_{-1}}$. This is computed as

$$
\begin{aligned}
n_{\mathbf{2 1}, \mathbf{2}-1}= & -\operatorname{dim} \mathbf{2}+\frac{1}{2} \cdot \frac{1}{8 \pi^{2}} \int F^{a} \wedge F^{b} \operatorname{Tr}_{\mathbf{2}} \tau^{a} \tau^{b} \\
& +\frac{1}{2} \cdot \frac{1}{8 \pi^{2}} \int F^{\mathrm{U}(1)} \wedge F^{\mathrm{U}(1)}(-1)^{2} \cdot 2 \\
= & -2+\frac{12+n-r}{2}+\frac{(-1)^{2}}{2} \cdot \frac{r}{14} \cdot 2 \\
= & \frac{1}{2}\left(n-\frac{6}{7} r+8\right) .
\end{aligned}
$$

Note that the 3rd (U(1) instanton) term is multiplied by 2 because each component of $\mathbf{2}_{-1}$ contributes to the index.

\footnotetext{
${ }^{6}$ The subscript "K3" for the integral is omitted below.
} 


\begin{tabular}{|c|c|}
\hline Representation & Multiplicity \\
\hline $\mathbf{4 8}$ & $-1(\mathrm{SU}(7)$ vector $)$ \\
$\mathbf{3 5}$ & $2 r^{\prime}-2$ \\
$\mathbf{2 1}$ & $n-6 r^{\prime}+8$ \\
$\mathbf{7}$ & $n+10 r^{\prime}+6$ \\
$\mathbf{1}$ & $2 n-14 r^{\prime}+20$ \\
\hline
\end{tabular}

Table 2. The spectrum for the configuration No.25.

Since $n_{\overline{\mathbf{2 1}, \mathbf{2}_{1}}}$ is equal to $n_{\mathbf{2 1 , \mathbf { 2 } _ { - 1 }}}(3.9)$, and there is no distinction between $\mathbf{2 1}$ and $\overline{\mathbf{2 1}}$ in six dimensions, the total multiplicity of $\mathbf{2 1}$ is in all

$$
n_{\mathbf{2 1}}=n-\frac{6}{7} r+8
$$

This becomes an integer if and only if the instanton number $r$ is a multiple of 7 . So writing $r=7 r^{\prime}$, we have

$$
n_{21}=n-6 r^{\prime}+8 .
$$

The computations of the multiplicities for other representations can be worked out similarly. The result is summarized in table 2 .

Finally, we would like to comment on the computation in [66] of the heterotic string matter spectrum with an $\mathrm{SU}(7)$ gauge group, which is different from ours but led them to the same result as that derived here. Since the multiplicities are not integers when $r$ is a multiple of 7 , they consider $\mathrm{U}(2)$ instead of $\mathrm{SU}(2)$ with an extra $\mathrm{U}(1)$ and assumed a contribution of this additional U(1) to the multiplicity of the singlet (See the top row of table 8 of [66], where the multiplicity of 1 contains the term $4 c_{2}\left(\mathcal{V}_{2}\right)-c_{1}(\mathcal{L})^{2}-6$ despite that the $\mathrm{SU}(7)$ singlets (the first two terms of (4.8) in [66]) are not charged under the $\mathrm{U}(1) \subset \mathrm{SU}(7) \times \mathrm{SU}(2) \times \mathrm{U}(1))$. This $\mathrm{U}(1)$ must commute with both the $\mathrm{SU}(7)$ and the $\mathrm{SU}(2)$ and must be different from the original $\mathrm{U}(1)$ factor in $E_{8}$, but obviously, there is no room for such an extra $\mathrm{U}(1)$ in $E_{8}$ as the rank is already exhausted.

\subsection{Orthogonal decompositions of the $E_{8}$ root lattice: why a multiple of seven?}

The fact that the $\mathrm{U}(1)$ instanton number must be a multiple of seven can be understood by an orthogonal decomposition of the $E_{8}$ root lattice. Let $\mathbf{e}_{i}(\mathrm{i}=1, \ldots, 9)$ be a set of orthonormal vectors of nine-dimensional flat Euclidean space with inner product

$$
\mathbf{e}_{i} \cdot \mathbf{e}_{j}=\delta_{i j}
$$

Then the following set of $72+84+84=240$ vectors on an eight-dimensional hyperplane normal to $\sum_{j=1}^{9} \mathbf{e}_{j}$ form the set of root vectors of $E_{8}$ :

$$
\begin{aligned}
& \mathbf{e}_{i}-\mathbf{e}_{j} \quad(1 \leq i \neq j \leq 9), \\
\pm & \left(\mathbf{e}_{i}+\mathbf{e}_{j}+\mathbf{e}_{k}-\frac{1}{3} \sum_{l=1}^{9} \mathbf{e}_{l}\right) \quad(1 \leq i<j<k \leq 9) .
\end{aligned}
$$


This fact can be most easily verified by considering Freudenthal's realization of the $E_{8}$ algebra [67,68]. The first line (3.13) is the set of root vectors of $\mathrm{SU}(9)$, while the second line (3.14) is the root vectors corresponding to the rank-3 tensors of $\mathrm{SU}(9)$. Using this presentation of $E_{8}$ roots, one can easily see where the roots of $\mathrm{SU}(7) \times \mathrm{SU}(2)$ are embedded and which root vector is the one corresponding to the $\mathrm{U}(1)$ generator. As the root vectors of $\mathrm{SU}(7)$ one can take

$$
\mathbf{e}_{i}-\mathbf{e}_{j} \quad(1 \leq i \neq j \leq 7),
$$

and the roots of $\mathrm{SU}(2) \subset H$ is then

$$
\pm\left(\mathbf{e}_{8}-\mathbf{e}_{9}\right) .
$$

In the $E_{8}$ root lattice generated by the vectors (3.13) and (3.14), the orthogonal lattice normal to the $\mathrm{SU}(7)$ and $\mathrm{SU}(2)$ lattices spanned by (3.15) and (3.16) is one-dimensional, generated by

$$
\frac{1}{3}\left(2 \sum_{l=1}^{7} \mathbf{e}_{l}-7\left(\mathbf{e}_{8}+\mathbf{e}_{9}\right)\right) .
$$

The length square of (3.17) is 14, which is 7 times as large as the simple-root length square. This explains why the instanton number of $U(1)$ is a multiple of 7 .

Note that the junction lattice [69], which is the inverse of the matrix of section parings, is given in the present case as

$$
\Lambda_{(25)}^{-1}=\left(\begin{array}{cr}
4 & -1 \\
-1 & 2
\end{array}\right) .
$$

This can be made diagonal by the change of basis

$$
\left(\begin{array}{ll}
2 & 1 \\
0 & 1
\end{array}\right)\left(\begin{array}{cr}
4 & -1 \\
-1 & 2
\end{array}\right)\left(\begin{array}{ll}
2 & 0 \\
1 & 1
\end{array}\right)=\left(\begin{array}{cc}
14 & 0 \\
0 & 2
\end{array}\right),
$$

which agrees with the above consideration.

\subsection{Example 2: $G=\mathrm{SU}(5) \times \mathrm{SU}(2)\left(T=A_{4} \oplus A_{1}\right)($ No.17$)$}

In this case, $\operatorname{rank}(E(K))=3$ and we can take one or two $\mathrm{U}(1)$ direction(s) in $H$. Namely, $H=\mathrm{SU}(3) \times \mathrm{U}(1)$ or $H=\mathrm{SU}(2) \times \mathrm{U}(1) \times \mathrm{U}(1)$. The gauge symmetry is the same $(G=\mathrm{SU}(5) \times \mathrm{SU}(2))$ for these two gauge bundles, but the resulting spectra are different. As we will explain below, the spectrum for $H=\mathrm{SU}(2) \times \mathrm{U}(1) \times \mathrm{U}(1)$ is more general than the one for $H=\mathrm{SU}(3) \times \mathrm{U}(1)$.

\subsubsection{The case when $H=\mathrm{SU}(2) \times \mathrm{U}(1) \times \mathrm{U}(1)$}

The junction lattice is

$$
\Lambda_{(17)}^{-1}=\left(\begin{array}{crr}
4 & -1 & 1 \\
-1 & 2 & -1 \\
1 & -1 & 2
\end{array}\right) .
$$

As $H$, we allow the instantons to live also in a different $\mathrm{U}(1)(\equiv \tilde{\mathrm{U}}(1))$ as well as the $\mathrm{SU}(2) \times \mathrm{U}(1)$ for No.25 in the previous section, so that the $\mathrm{SU}(7)$ is further broken to 


\begin{tabular}{|c|c|c|c|}
\hline Rep. of $\mathrm{SU}(5) \times \mathrm{SU}(2)$ & Rep. of $\mathrm{SU}(2)$ (in $H$ ) & $\mathrm{U}(1)$ charge & $\tilde{\mathrm{U}}(1)$ charge \\
\hline$(24,1)$ & 1 & 0 & 0 \\
\hline$(\mathbf{1}, \mathbf{3})$ & 1 & 0 & 0 \\
\hline$(5,2)$ & 1 & 0 & 7 \\
\hline$(\overline{\mathbf{5}}, 2)$ & 1 & 0 & -7 \\
\hline$(\mathbf{1}, \mathbf{1})(\tilde{\mathrm{U}}(1))$ & 1 & 0 & 0 \\
\hline$(5,1)$ & 2 & 3 & 2 \\
\hline$(5,1)$ & 1 & -4 & 2 \\
\hline$(1,2)$ & 2 & 3 & -5 \\
\hline$(\mathbf{1}, \mathbf{2})$ & 1 & -4 & -5 \\
\hline$(\overline{5}, \mathbf{1})$ & 2 & -3 & -2 \\
\hline$(\overline{\mathbf{5}}, \mathbf{1})$ & 1 & 4 & -2 \\
\hline$(\mathbf{1}, \mathbf{2})$ & 2 & -3 & 5 \\
\hline$(1,2)$ & 1 & 4 & 5 \\
\hline$(\overline{\mathbf{1 0}}, \mathbf{1})$ & 1 & 2 & 6 \\
\hline$(10,2)$ & 1 & 2 & -1 \\
\hline$(5, \mathbf{1})$ & 1 & 2 & -8 \\
\hline$(10,1)$ & 1 & -2 & -6 \\
\hline$(\overline{\mathbf{1 0}}, 2)$ & 1 & -2 & 1 \\
\hline$(\overline{\mathbf{5}}, \mathbf{1})$ & 1 & -2 & 8 \\
\hline$(10,1)$ & 2 & -1 & 4 \\
\hline$(5,2)$ & 2 & -1 & -3 \\
\hline$(\mathbf{1}, \mathbf{1})$ & 2 & -1 & -10 \\
\hline$(\overline{\mathbf{1 0}}, \mathbf{1})$ & 2 & 1 & -4 \\
\hline$(\overline{\mathbf{5}}, \mathbf{2})$ & 2 & 1 & 3 \\
\hline$(\overline{\mathbf{1}}, \mathbf{1})$ & 2 & 1 & 10 \\
\hline$(\mathbf{1}, \mathbf{1})(\mathrm{U}(1))$ & 1 & 0 & 0 \\
\hline$(\mathbf{1}, \mathbf{1})$ & 3 & 0 & 0 \\
\hline
\end{tabular}

Table 3. Decomposition of 248.

$\mathrm{SU}(5) \times \mathrm{SU}(2)$. The instanton numbers are assumed to be $12+n-r-\tilde{r}, r$ and $\tilde{r}$ for $\mathrm{SU}(2), \mathrm{U}(1)$ and $\tilde{\mathrm{U}}(1)$, respectively. The decomposition of $\mathbf{2 4 8}$ in representations of these subgroups is shown in table 3.

In the present case

$$
\sum_{E_{8}}(\mathrm{U}(1) \text { charge })^{2}=14 \cdot 60
$$

and

$$
\sum_{E_{8}}(\tilde{\mathrm{U}}(1) \text { charge })^{2}=70 \cdot 60
$$




\begin{tabular}{|c|c|}
\hline Representation & Multiplicity \\
\hline$(\mathbf{2 4}, \mathbf{1})$ & $-1(\mathrm{SU}(5)$ vector $)$ \\
$(\mathbf{1}, \mathbf{3})$ & $-1(\mathrm{SU}(2)$ vector $)$ \\
$(\mathbf{1}, \mathbf{1})$ & $3 n+27-10\left(\frac{2}{7} r+\frac{1}{70} \tilde{r}\right)$ \\
$(\mathbf{5}, \mathbf{2})$ & $n+6-3\left(\frac{2}{7} r+\frac{1}{70} \tilde{r}\right)$ \\
$(\mathbf{5}, \mathbf{1})$ & $n+4+6\left(\frac{2}{7} r+\frac{1}{70} \tilde{r}\right)$ \\
$(\mathbf{1}, \mathbf{2})$ & $n+6+5\left(\frac{2}{7} r+\frac{1}{70} \tilde{r}\right)$ \\
$(\mathbf{1 0}, \mathbf{2})$ & $-2+\left(\frac{2}{7} r+\frac{1}{70} \tilde{r}\right)$ \\
$(\mathbf{1 0}, \mathbf{1})$ & $n+6-2\left(\frac{2}{7} r+\frac{1}{70} \tilde{r}\right)$ \\
\hline
\end{tabular}

Table 4. The spectrum for the configuration No.17.

so that

$$
\begin{aligned}
\frac{1}{8 \pi^{2}} \int F^{a} \wedge F^{b} \operatorname{Tr} \tau^{a} \tau^{b} & =12+n-r-\tilde{r}, \\
\frac{1}{8 \pi^{2}} \int F^{\mathrm{U}(1)} \wedge F^{\mathrm{U}(1)} & =\frac{r}{14}, \\
\frac{1}{8 \pi^{2}} \int F^{\tilde{\mathrm{U}}(1)} \wedge F^{\tilde{\mathrm{U}}(1)} & =\frac{\tilde{r}}{70} .
\end{aligned}
$$

The spectrum for No.17 is similarly obtained as shown in table 4.

For $\frac{2}{7} r+\frac{1}{70} \tilde{r}$ to be integer, the general solution is

$$
(r, \tilde{r})=(3,10) k+(-1,20) l \quad(k, l \in \mathbb{Z}),
$$

so that

$$
\frac{2}{7} r+\frac{1}{70} \tilde{r}=k \in \mathbb{Z},
$$

therefore $r$ or $\tilde{r}$ need not necessarily be a multiple of 7 or 70 in general. The orthogonal decomposition of the junction lattice is

$$
\left(\begin{array}{rrr}
2 & 1 & 0 \\
0 & 1 & 0 \\
1 & -3 & -7
\end{array}\right)\left(\begin{array}{rrr}
4 & -1 & 1 \\
-1 & 2 & -1 \\
1 & -1 & 2
\end{array}\right)\left(\begin{array}{rrr}
2 & 0 & 1 \\
1 & 1 & -3 \\
0 & 0 & -7
\end{array}\right)=\left(\begin{array}{ccc}
14 & 0 & 0 \\
0 & 2 & 0 \\
0 & 0 & 70
\end{array}\right)
$$

which implies that the minimal $\tilde{U}(1)$ charge square (measured by the $E_{8}$ root space inner product) is 35 times (and not 70 times) as large as that of the $\mathrm{SU}(2)$ factor. This is almost equivalent to the condition for the integrality of the multiplicities, but the latter is "twice as" severe.

\subsubsection{The case when $H=\mathrm{SU}(3) \times \mathrm{U}(1)$}

We can see the $\mathrm{SU}(3)$ Cartan matrix in the right-down block of

$$
\Lambda_{(17)}^{-1}=\left(\begin{array}{crr}
4 & -1 & 1 \\
-1 & 2 & -1 \\
1 & -1 & 2
\end{array}\right)
$$




\begin{tabular}{|c|cc|}
\hline Rep. of SU(5) $\times \mathrm{SU}(2)$ & Rep. of SU(3) & $\mathrm{U}(1)_{(17)}$ charge \\
\hline$(\mathbf{2 4}, \mathbf{1})$ & $\mathbf{1}$ & 0 \\
$(\mathbf{1}, \mathbf{3})$ & $\mathbf{1}$ & 0 \\
$(\mathbf{1}, \mathbf{2})$ & $\mathbf{3}$ & 5 \\
$(\mathbf{1}, \mathbf{2})$ & $\overline{\mathbf{3}}$ & -5 \\
$(\mathbf{1}, \mathbf{1})$ & $\mathbf{8}$ & 0 \\
$(\mathbf{1}, \mathbf{1})$ & $\mathbf{1}$ & 0 \\
$(\mathbf{5}, \mathbf{2})$ & $\overline{\mathbf{3}}$ & \\
$(\mathbf{5}, \mathbf{1})$ & $\mathbf{3}$ & -4 \\
$(\mathbf{5}, \mathbf{1})$ & $\mathbf{1}$ & 6 \\
$(\overline{\mathbf{5}}, \mathbf{2})$ & $\mathbf{3}$ & -1 \\
$(\overline{\mathbf{5}}, \mathbf{1})$ & $\overline{\mathbf{3}}$ & 4 \\
$(\overline{\mathbf{5}}, \mathbf{1})$ & $\mathbf{1}$ & -6 \\
$(\mathbf{1 0}, \mathbf{2})$ & & \\
$(\mathbf{1 0}, \mathbf{1})$ & $\mathbf{1}$ & 3 \\
$(\overline{\mathbf{1 0}}, \mathbf{2})$ & $\overline{\mathbf{3}}$ \\
$(\overline{\mathbf{1 0}}, \mathbf{1})$ & $\mathbf{1}$ & -2 \\
& $\mathbf{3}$ & -3 \\
\hline
\end{tabular}

Table 5. Decomposition of $\mathbf{2 4 8 .}$

and we can also take $\mathrm{SU}(3) \times \mathrm{U}(1)\left(\equiv \mathrm{U}(1)_{(17)}\right)$ as $H$. Orthogonal decomposition of the junction lattice is

$$
\left(\begin{array}{ccc}
3 & 1 & -1 \\
0 & 1 & 0 \\
0 & 0 & 1
\end{array}\right)\left(\begin{array}{crr}
4 & -1 & 1 \\
-1 & 2 & -1 \\
1 & -1 & 2
\end{array}\right)\left(\begin{array}{ccc}
3 & 0 & 0 \\
1 & 1 & 0 \\
-1 & 0 & 1
\end{array}\right)=\left(\begin{array}{ccc}
30 & 0 & 0 \\
0 & 2 & -1 \\
0 & -1 & 2
\end{array}\right) .
$$

In representations of this $G \times H$, the $E_{8}$ adjoint is decomposed as shown in table 5 . We assume $12+n-r_{(17)}$ instantons in $\mathrm{SU}(3)$ and $r_{(17)}$ in $\mathrm{U}(1)_{(17)}$. Then since

$$
\sum_{E_{8}}\left(\mathrm{U}(1)_{(17)} \text { charge }\right)^{2}=30 \cdot 60
$$

we obtain

$$
\begin{aligned}
\frac{1}{8 \pi^{2}} \int F^{a} & \wedge F^{b} \operatorname{Tr} \lambda^{a} \lambda^{b}=12+n-r_{(17)}, \\
\frac{1}{8 \pi^{2}} \int F^{\mathrm{U}(1)_{(17)}} \wedge F^{\mathrm{U}(1)_{(17)}} & =\frac{r_{(17)}}{30},
\end{aligned}
$$

where $\lambda^{a}(a=1, \ldots, 8)$ are the Gell-Mann matrices. Incidentally, $\mathrm{U}(1)_{(17)}$ is the "hypercharge" $\mathrm{U}(1)$ that breaks one of $\mathrm{SU}(5)$ of $\mathrm{SU}(5) \times \mathrm{SU}(5) \subset E_{8}$ into $\mathrm{SU}(3) \times \mathrm{SU}(2)$. The spectrum is as shown in table 6 . 


\begin{tabular}{|c|c|}
\hline Representation & Multiplicity \\
\hline$(\mathbf{2 4}, \mathbf{1})$ & $-1(\mathrm{SU}(5)$ vector $)$ \\
$(\mathbf{1}, \mathbf{3})$ & $-1(\mathrm{SU}(2)$ vector $)$ \\
$(\mathbf{1}, \mathbf{1})$ & $3 n+27-30 r_{(17)}^{\prime}$ \\
$(\mathbf{5}, \mathbf{2})$ & $n+6-9 r_{(17)}^{\prime}$ \\
$(\mathbf{5}, \mathbf{1})$ & $n+4+18 r_{(17)}^{\prime}$ \\
$(\mathbf{1}, \mathbf{2})$ & $n+6+15 r_{(17)}^{\prime}$ \\
$(\mathbf{1 0}, \mathbf{2})$ & $-2+3 r_{(17)}^{\prime}$ \\
$(\mathbf{1 0}, \mathbf{1})$ & $n+6-6 r_{(17)}^{\prime}$ \\
\hline
\end{tabular}

Table 6. The spectrum for the configuration No.17. Another derivation.

Here we have defined $r_{(17)}=10 r_{(17)}^{\prime}$ so that the multiplicities become all integers when the $\mathrm{U}(1)_{(17)}$ instanton number $r_{(17)}$ is a multiple of 10 (which is when $r_{(17)}^{\prime}$ is an integer).

Note that this spectrum coincides with the spectrum shown in table 4 provided that the replacement

$$
\frac{2}{7} r+\frac{1}{70} \tilde{r}=k=3 r_{(17)}^{\prime}
$$

is made. Thus the spectrum for $H=\mathrm{SU}(3) \times \mathrm{U}(1)$ is "three times" as restrictive as that for $H=\mathrm{SU}(2) \times \mathrm{U}(1)^{2}$.

The index computations for other cases can be done similarly. We summarize the relevant results (No.19 and No.12 for SU(7) series and No.29 and No.22 for SU(8) series) in appendices $\mathrm{A}$ and $\mathrm{B}$.

\section{Geometries for non-Cartan Mordell-Weil lattices}

Suppose that a non-Cartan Mordell-Weil lattice $E(K)$ (non-Cartan gauge bundle $H$ ) is given. To construct the corresponding geometry, which is a rational elliptic surface fibered over $\mathbb{P}^{1}$, we first construct a $\mathrm{K} 3$-fibered $\mathrm{CY}_{3}$ with the singularity $T$ (gauge symmetry $G$ ) paired with the given $E(K)$. Then, we map the $\mathrm{CY}_{3}$ to a RES-fibered geometry.

\section{1 $\mathrm{CY}_{3}$ in six-dimensional F-theory/heterotic duality}

We first review the construction of a $\mathrm{CY}_{3}$ for six-dimensional F-theory compactification $[2$, $3,70] . E_{8} \times E_{8}$ heterotic string/K3 contains 24 instantons. Suppose $12+n$ of them take values in a gauge bundle $H$ in the first $E_{8}$ and the gauge symmetry is broken to $G$, while the other $12-n$ instantons take values in another gauge bundle $H^{\prime}$ in the second $E_{8}$ and the gauge symmetry is broken to $G^{\prime}$. It has been known [2] that the corresponding dual F-theory geometry is a $\mathrm{K} 3$-fibered $\mathrm{CY}_{3}$ over $\mathbb{P}^{1}$ and at the same time is an elliptic fibered $\mathrm{CY}_{3}$ over the Hirzebruch surface $F_{n}$ with singularities $G$ and $G^{\prime}$. The Weierstrass form is given by

$$
0=y^{2}+x^{3}+f\left(z, z^{\prime}\right) x+g\left(z, z^{\prime}\right)
$$




\begin{tabular}{|rrccc|}
\hline $\operatorname{ord}(f)$ & ord $(g)$ & ord $(\Delta)$ & Fiber type & Singularity type \\
\hline$\geq 0$ & $\geq 0$ & 0 & smooth & none \\
0 & 0 & $n$ & $I_{n}$ & $A_{n-1}$ \\
$\geq 1$ & 1 & 2 & $I I$ & none \\
1 & $\geq 2$ & 3 & $I I I$ & $A_{1}$ \\
$\geq 2$ & 2 & 4 & $I V$ & $A_{2}$ \\
2 & $\geq 3$ & $n+6$ & $I_{n}^{*}$ & $D_{n+4}$ \\
$\geq 2$ & 3 & $n+6$ & $I_{n}^{*}$ & $D_{n+4}$ \\
$\geq 3$ & 4 & 8 & $I V^{*}$ & $E_{6}$ \\
3 & $\geq 5$ & 9 & $I I I^{*}$ & $E_{7}$ \\
$\geq 4$ & 5 & 10 & $I I^{*}$ & $E_{8}$ \\
$\geq 4$ & $\geq 6$ & $\geq 12$ & non-min & - \\
\hline
\end{tabular}

Table 7. The Kodaira classification of singularities.

with

$$
\begin{aligned}
& f\left(z, z^{\prime}\right)=\sum_{i=0}^{8} f_{8+(4-i) n} z^{i}, \\
& g\left(z, z^{\prime}\right)=\sum_{j=0}^{12} g_{12+(6-j) n} z^{j} .
\end{aligned}
$$

$F_{n}$ is a $\mathbb{P}^{1}$ fibration over the base $\mathbb{P}^{1}$, whose coordinates are $z$ and $z^{\prime}$, respectively. $f_{k}$ and $g_{k}$ are polynomials of $z^{\prime}$ with homogeneous order $k$. Let us write the fiber coordinate $z$ in a homogeneous form $(u: v)$. Under the $C^{*}$ action $(u: v) \rightarrow(\mu u: \mu v)$, the other coordinates transform as

$$
\left(x, y, z^{\prime}\right) \rightarrow\left(\mu^{4} x, \mu^{6} y, z^{\prime}\right) .
$$

Also, for the base coordinate $z^{\prime} \equiv\left(u^{\prime}: v^{\prime}\right)$, the $C^{*}$ action $\left(u^{\prime}: v^{\prime}\right) \rightarrow\left(\lambda u^{\prime}: \lambda v^{\prime}\right)$ gives

$$
(x, y, z) \rightarrow\left(\lambda^{4+2 n} x, \lambda^{6+3 n} y, \lambda^{n} z\right) .
$$

The Weierstrass form (4.1) is homogeneous under these $C^{*}$ actions with degrees 12 and $12+6 n$.

Singularities $G$ and $G^{\prime}$ are realized by demanding that $f, g$ and the discriminant $\Delta$,

$$
\Delta\left(z, z^{\prime}\right)=4 f\left(z, z^{\prime}\right)^{3}+27 g\left(z, z^{\prime}\right)^{2},
$$

have suitable vanishing orders near $z=0$ and $1 / z=0$ (see table 7 ).

For realizing $A D E$ gauge symmetry in six dimensions, these Kodaira fibers should satisfy the so-called split conditions [70], which we will require in our construction since all the gauge symmetries that we would like to achieve (table 1 ) are of the $A$ type. (The explicit form of the split condition can be seen in table 8 below.)

The last row of table 7 expresses the singularity that cannot be resolved by any blow up of the fiber. To resolve it, blowing up the base is required and an additional tensor 
multiplet appears in the spectrum. Such a theory does not have a heterotic dual in the perturbative regime, and hence is not the subject of our study.

The elliptic fibration over $F_{n}$ can also be written in Tate's form [70, 71]

$$
0=y^{2}+x^{3}+a_{1} x y+a_{2} x^{2}+a_{3} y+a_{4} x+a_{6},
$$

where $a_{i}$ are polynomials of $z$ and $z^{\prime}$. Because of the homogeneity under (4.3), $a_{i}$ are degree $2 i$ polynomials in $z$ :

$$
a_{i}\left(z, z^{\prime}\right)=\sum_{j=0}^{2 i} a_{i j} z^{j} .
$$

Here $a_{i j}$ are polynomials of $z^{\prime}$. Their degrees are determined by the homogeneity under (4.4) as

$$
\operatorname{deg}\left(a_{i j}\right)=2 i+(i-j) n
$$

Discriminant $\Delta$ is given by

$$
\Delta=-b_{2}^{2} b_{8}+27 b_{6}^{2}+4 b_{4}^{3}-18 b_{2} b_{4} b_{6},
$$

where $b_{n}(n=2,4,6,8)$ are defined in terms of $a_{i}$ as follows:

$$
\begin{aligned}
& b_{2}=-\frac{a_{1}^{2}}{4}+a_{2}, \\
& b_{4}=-\frac{a_{1} a_{3}}{2}+a_{4}, \\
& b_{6}=a_{6}-\frac{a_{3}^{2}}{4}, \\
& b_{8}=b_{4}^{2}-4 b_{2} b_{6} .
\end{aligned}
$$

Singularities $G$ and $G^{\prime}$ are realized by requiring $a_{i}(i=1,2,3,4,6)$ to vanish at suitable orders of $z$, known as Tate's algorithm (see table 8). The vanishing orders of $a_{i}$ not only determine the type of the local singularity but also control its global structure. We listed in table 8 the fibers for which the split condition is satisfied (the subscript "s" is attatched ${ }^{7}$ ). The first six columns of the table represent the lowest orders of $a_{i}$ and $\Delta$ in $z$. The next column is the coefficient of $z^{\text {ord( }(\Delta)}$, where $b_{n j}$ (or $b_{n, j}$ ) represents the coefficient of $z^{j}$ in $b_{n}$. The "split conditions" for Kodaira fibers are the conditions that the discriminants (4.5) should be the same forms as in this column. Last two columns are the corresponding fiber degeneracy and the singularity type. ${ }^{8}$ The last row of the table expresses the singularity that never be resolved by blowing up the fiber.

\footnotetext{
${ }^{7}$ The fibers without subscript "s" in the table have at most only one exceptional divisor, and hence are necessarily of the split type.

${ }^{8}$ To realize the groups with dagger, one more condition should be fulfilled. There are some polynomials $p$ and $q$ such that (here $a_{n, j}$ denotes $a_{n j}$ )

$$
\begin{aligned}
\mathrm{SO}(8) & : a_{21}^{2}-4 a_{42}=p^{2}, \\
\mathrm{SO}(4 k+4) & : a_{4, k+1}^{2}-4 a_{21} a_{6,2 k+1}=q^{2} .
\end{aligned}
$$
}




\begin{tabular}{|lllll|ll|ll|}
\hline $\operatorname{ord}\left(a_{1}\right)$ & $\operatorname{ord}\left(a_{2}\right)$ & $\operatorname{ord}\left(a_{3}\right)$ & $\operatorname{ord}\left(a_{4}\right)$ & $\operatorname{ord}\left(a_{6}\right)$ & $\operatorname{ord}(\Delta)$ & Coefficient of $z^{\operatorname{ord}(\Delta)}$ & Fiber & Group \\
\hline 0 & 0 & 1 & 1 & 1 & 1 & $4 b_{20}^{3} a_{61}$ & $I_{1}$ & - \\
0 & 0 & 1 & 1 & 2 & 2 & $-b_{20}^{2} b_{82}$ & $I_{2}$ & $\mathrm{SU}(2)$ \\
0 & 1 & 1 & 2 & 3 & 3 & $\frac{1}{16} a_{10}^{3}\left(a_{31}^{3}-a_{10} b_{83}\right)$ & $I_{3}^{\mathrm{s}}$ & $\mathrm{SU}(3)$ \\
0 & 1 & $k$ & $k$ & $2 k$ & $2 k$ & $-\frac{1}{16} a_{10}^{4} b_{8,2 k}$ & $I_{2 k}^{\mathrm{s}}$ & $\mathrm{SU}(2 k)$ \\
0 & 1 & $k$ & $k+1$ & $2 k+1$ & $2 k+1-\frac{1}{16} a_{10}^{4} b_{8,2 k+1}$ & $I_{2 k+1}^{\mathrm{s}}$ & $\mathrm{SU}(2 k+1)$ \\
\hline 1 & 1 & 1 & 1 & 1 & 2 & $27 a_{61}^{2}$ & $I I$ & - \\
1 & 1 & 1 & 1 & 2 & 3 & $4 a_{41}^{3}$ & $I I I$ & $\mathrm{SU}(2)$ \\
1 & 1 & 1 & 2 & 3 & 4 & $\frac{27}{16} a_{31}^{4}$ & $I V^{\mathrm{s}}$ & $\mathrm{SU}(3)$ \\
1 & 1 & 2 & 2 & 4 & 6 & $-\frac{1}{16} p^{2}\left(p+a_{21}\right)^{2}\left(p-a_{21}\right)^{2}$ & $I_{0}^{* \mathrm{~s}}$ & $\mathrm{SO}(8)^{\dagger}$ \\
1 & 1 & $k$ & $k+1$ & $2 k+1$ & $2 k+3$ & $-a_{21}^{3} a_{3 k}^{2}$ & $I_{2 k-3}^{*}$ & $\mathrm{SO}(4 k+2)$ \\
1 & 1 & $k+1$ & $k+1$ & $2 k+1$ & $2 k+4$ & $-q^{2} a_{21}^{2}$ & $I_{2 k-2}^{*}$ & $\mathrm{SO}(4 k+4)^{\dagger}$ \\
1 & 2 & 2 & 3 & 5 & 8 & $\frac{27}{16} a_{32}^{4}$ & $I V^{* \mathrm{~s}}$ & $E_{6}$ \\
1 & 2 & 3 & 3 & 5 & 9 & $4 a_{43}^{3}$ & $I I I^{* \mathrm{~s}}$ & $E_{7}$ \\
1 & 2 & 3 & 4 & 5 & 10 & $27 a_{65}^{2}$ & $I I^{* \mathrm{~s}}$ & $E_{8}$ \\
1 & 2 & 3 & 4 & 6 & 12 & {$\left[\Delta / z^{12}\right]_{z=0}$} & non-min & - \\
\hline
\end{tabular}

Table 8. Tate's algorithm.

The relation between the Weierstrass form (4.2) and Tate's form (4.7) is given by

$$
\begin{aligned}
& f=-\frac{b_{2}^{2}}{3}+b_{4}, \\
& g=\frac{2}{27} b_{2}^{3}-\frac{b_{4} b_{2}}{3}+b_{6} .
\end{aligned}
$$

\subsection{Mapping $\mathrm{CY}_{3}$ to RES-fibered geometry}

The Weierstrass form of a rational elliptic surface is given by

$$
0=y^{2}+x^{3}+f(z) x+g(z),
$$

where $f$ and $g$ are sections of $\mathcal{O}(4)$ and $\mathcal{O}(6)$ on the base $\mathbb{P}^{1}$ and have the form

$$
f(z)=\sum_{i=0}^{4} f_{i} z^{i}, \quad g(z)=\sum_{j=0}^{6} g_{j} z^{j} .
$$

Suppose that we are given a $\mathrm{CY}_{3}$ with the Weierstrass form (4.1), whose $f$ and $g$ are sections of $\mathcal{O}(8)$ and $\mathcal{O}(12)$ on $\mathbb{P}^{1}$ as in (4.2), yielding a $\mathrm{K} 3$ fibration. By using their coefficients $f_{8+(4-i) n}$ for $i \leq 4$ and $g_{12+(6-j) n}$ for $j \leq 6$, one can construct a geometry with a Weierstrass form

$$
0=y^{2}+x^{3}+f^{\prime}\left(z, z^{\prime}\right) x+g^{\prime}\left(z, z^{\prime}\right)
$$


where

$$
\begin{aligned}
& f^{\prime}\left(z, z^{\prime}\right)=\sum_{i=0}^{4} f_{8+(4-i) n} z^{i}, \\
& g^{\prime}\left(z, z^{\prime}\right)=\sum_{j=0}^{6} g_{12+(6-j) n} z^{j}
\end{aligned}
$$

with discriminant

$$
\Delta^{\prime}=4 f^{\prime 3}+27 g^{\prime 2}
$$

Here we regard $f^{\prime}$ and $g^{\prime}$ as sections of $\mathcal{O}(4)$ and $\mathcal{O}(6)$ on $\mathbb{P}^{1}$ (with coordinate $z$ ). Then the resulting geometry is a rational elliptic surface fibered over $\mathbb{P}^{1}$ (with coordinate $z^{\prime}$ ). This gives the map from the K3-fibered $\mathrm{CY}_{3}$ to a RES-fibered geometry:

$$
\mathrm{CY}_{3} \rightarrow \text { RES-fibered geometry. }
$$

When the rank of $G$ is large, we have to do a slight modification to $f^{\prime}$ and $g^{\prime}$ in order that the map does not change the singularity $G$. For example, for $G=\mathrm{SU}(n)$ with $n \leq 5$, the above procedure maps $G$ of a $\mathrm{CY}_{3}$ to the same $G$ in a RES-fibered geometry, but for $G=\mathrm{SU}(6)$, the naïve mapping changes the singularity. The explicit form of a $\mathrm{CY}_{3}$ with $G=\mathrm{SU}(6)$ is given by [66]

$$
\begin{aligned}
f= & -\frac{\alpha^{4} \beta^{4}}{48}-\frac{\alpha^{2} \beta^{3}}{6} \nu z-\frac{\beta}{6}\left(\alpha^{2} \phi_{2}+2 \beta \nu^{2}\right) z^{2}-\left(3 \beta \lambda+\frac{1}{3} \phi_{2} \nu\right) z^{3}+f_{8} z^{4} \\
& +f_{8-n} z^{5}+\cdots f_{8-4 n} z^{8}, \\
g= & \frac{\alpha^{6} \beta^{6}}{864}+\frac{\alpha^{4} \beta^{5}}{72} \nu z+\frac{\alpha^{2} \beta^{3}}{72}\left(\alpha^{2} \phi_{2}+4 \beta \nu^{2}\right) z^{2}+\frac{\beta^{2}}{108}\left(8 \beta \nu^{3}+9 \alpha^{2}\left(3 \beta \lambda+\phi_{2} \nu\right)\right) z^{3} \\
& +\frac{1}{36}\left(\alpha^{2}\left(\phi_{2}^{2}-3 \beta^{2} f_{8}\right)+4 \beta \nu\left(9 \beta \lambda+\phi_{2} \nu\right)\right) z^{4}+\frac{1}{12}\left(12 \phi_{2} \lambda-4 \beta \nu f_{8}-\alpha^{2} \beta^{2} f_{8-n}\right) z^{5}+g_{12} z^{6} \\
& +g_{12-n} z^{7}+\cdots+g_{12-6 n} z^{12} .
\end{aligned}
$$

In this $\mathrm{CY}_{3}, \operatorname{ord}(\Delta)=6$ and the gauge symmetry is $\mathrm{SU}(6)$. After mapping to $f^{\prime}$ and $g^{\prime}$ and calculating $\Delta^{\prime}$, one can see that $\operatorname{ord}\left(\Delta^{\prime}\right)=5$, i.e., the singularity is reduced by the map. The source of this reduction is $f_{8-n}$. Although $f_{8-n}$ is the coefficient of the term higher than $o\left(z^{4}\right)$ in $f$, it also appears in the $o\left(z^{5}\right)$ term in $g$. Thus, after the map $g \rightarrow g^{\prime}$, it is still contained in $g^{\prime}$. By setting this polynomial to zero in $g^{\prime}$, one recovers $\operatorname{ord}\left(\Delta^{\prime}\right)=6$ and the singularity remains to be $\mathrm{SU}(6)$.

In summary, the map (4.17) is obtained by first replacing $f$ and $g$ to $f^{\prime}$ and $g^{\prime}$, and then regarding $f^{\prime}$ and $g^{\prime}$ as sections of $\mathcal{O}(4)$ and $\mathcal{O}(6)$ of $\mathbb{P}^{1}$, and finally setting to zero the polynomials which constitute the coefficients of the terms higher than $o\left(z^{4}\right)$ in $f$ and $o\left(z^{6}\right)$ in $g$ and are still contained in $f^{\prime}$ and $g^{\prime}$ even after the map $f, g \rightarrow f^{\prime}, g^{\prime}$. Hereafter, the resulting $f^{\prime}, g^{\prime}$ and $\Delta^{\prime}$ will be denoted by $f_{\text {res }}, g_{\text {res }}$ and $\Delta_{\text {res }}$.

This last step, however, does not work for some $\mathrm{CY}_{3}$ with sufficiently large rank $G$. In such cases, one cannot recover the original singularity $G$ of $\mathrm{CY}_{3}$ in the RES-fibered geometry and the map (4.17) does change the singularity. 
There are two such cases. In the first case, additional singularities other than $G$ are produced by the map. As an example, let us consider a $\mathrm{CY}_{3}$ with $G=\mathrm{SU}(8)$ constructed by using Tate's algorithm. The orders of $a_{i}$ in $(4.7)$ are set to be $\operatorname{ord}\left(a_{i}\right)=(0,1,4,4,8)$ (see table 8). Its Weierstrass form is obtained by using (4.10) and (4.11). As a result, $\operatorname{ord}(\Delta)=8$ is realized. Mapping to $f^{\prime}$ and $g^{\prime}$, one finds that $\operatorname{ord}\left(\Delta^{\prime}\right)=5$. One can see that $f^{\prime}$ and $g^{\prime}$ contain the following polynomials which constitute the coefficients of the terms higher than $o\left(z^{4}\right)$ in $f$ and $o\left(z^{6}\right)$ in $g$ :

$$
a_{12}, a_{23}, a_{24}, a_{34}, a_{35}, a_{36}, a_{45}, a_{46} .
$$

Setting these polynomials to zero, one recovers ord $\left(\Delta_{\text {res }}\right)=8$, but this $\mathrm{SU}(8)$ singularity is accompanied by additional singularities. Explicitly, $\Delta_{\text {res }}$ has a factorized form

$$
\Delta_{\text {res }}=a_{44}^{2} z^{8} D_{8+4 n}
$$

with

$$
\begin{aligned}
D_{8+4 n}= & -\frac{1}{16}\left[a_{10}^{4}+4 a_{10}^{2}\left(a_{10} a_{11}-2 a_{21}\right) z+\left\{a_{10}^{2}\left(6 a_{11}^{2}-8 a_{22}\right)-16 a_{21}\left(a_{10} a_{11}-a_{21}\right)\right\} z^{2}\right. \\
& \left.+4\left(a_{11}^{2}-4 a_{22}\right)\left(a_{10} a_{11}-2 a_{21}\right) z^{3}+\left\{a_{11}^{2}\left(a_{11}^{2}-8 a_{22}\right)+16\left(a_{22}^{2}-4 a_{44}\right)\right\} z^{4}\right] .
\end{aligned}
$$

Since the degree of $a_{44}$ is 8 , we can write $a_{44}\left(z^{\prime}\right)=\prod_{i=1}^{8}\left(z^{\prime}-z_{i}^{\prime}\right)$. Then we obtain

$$
\Delta_{\mathrm{res}}=z^{8} \prod_{i=1}^{8}\left(z^{\prime}-z_{i}^{\prime}\right)^{2} D_{8+4 n} .
$$

At $z^{\prime}=z_{i}^{\prime}, \operatorname{ord}\left(\Delta_{\text {res }}\right)$ in $z^{\prime}$ is enhanced to 2 . Also, one can see that $\operatorname{ord}\left(f_{\text {res }}\right)=\operatorname{ord}\left(g_{\text {res }}\right)=0$ at these loci, yielding $I_{2}$ fibers. Thus an additional gauge symmetry $\mathrm{SU}(2)$ appears along each line $z^{\prime}=z_{i}^{\prime}$ perpendicular to $z=0$ where the $\mathrm{SU}(8)$ singularity exists. To explain the origin of such additional singularities, let us go back to Tate's algorithm and focus on the orders of $a_{3}$ and $a_{6}$, which are 4 and 8 for $G=\mathrm{SU}(8)$. It means that $a_{3}(z)$ contains $a_{34}, a_{35}, a_{36}$ and $a_{6}(z)$ contains $a_{68}, a_{69}, \ldots, a_{6,12}$. In $f^{\prime}$ and $g^{\prime}, a_{6 j}$ are not contained since they only appear in $f$ and $g$ in the terms higher than $o\left(z^{4}\right)$ and $o\left(z^{6}\right)$. Also, $f_{\text {res }}$ and $g_{\text {res }}$ do not contain $a_{3 j}$, since they are set to zero (4.19). It means $a_{3}(z)=a_{6}(z)=0$ in $f_{\text {res }}$ and $g_{\text {res. }}$ Then, from (4.9) and (4.10), discriminant has a factorized form $\Delta_{\text {res }}=a_{4}^{2}\left(4 a_{4}-b_{2}^{2}\right)$, leading to unwanted additional singularities (4.20). In general, when the orders of $a_{3}$ and $a_{6}$ in Tate's algorithm exceed 3 and 6 simultaneously, additional singularities appear in the resulting RES-fibered geometry.

In the second case, the map (4.17) changes the singularity type of the fiber at $z=0$. This occurs when the singularity $G$ in a $\mathrm{CY}_{3}$ is not contained within one $E_{8}(z=0)$ but spreads into the other $E_{8}(z=\infty)$. For example, let us consider $G=\mathrm{SU}(8)$ again, but take another $\mathrm{CY}_{3}$ which is different from the one obtained from Tate's algorithm presented above and is constructed by working in the Weierstrass form directly. As seen from (4.11), a $\mathrm{CY}_{3}$ in Tate's form is always rewritten in the Weierstrass form, but the inverse is not 
the case in general. This means that there may exist models that are described only in the Weierstrass form and have no Tate's counterpart (see e.g., [72]). Hence the appearance of additional singularities (4.22) in RES-fibered geometry may be an artifact in Tate's form. There may exist another SU(8) model that cannot be reached by Tate's algorithm and for that $\mathrm{CY}_{3}$ additional singularity may not arise after the map (4.17). A candidate of such a $\mathrm{CY}_{3}$ is the one constructed in [66]. The explicit form is given by ${ }^{9}$

$$
\begin{aligned}
f= & -\frac{1}{48} \tau^{4}-\frac{1}{6} \tau^{2} \zeta_{3} z-\frac{1}{6}\left(2 \zeta_{3}^{2}+\tau^{2} \omega_{1}\right) z^{2}-\frac{1}{3}\left(9 \tau^{2} \lambda_{2}+2 \zeta_{3} \omega_{1}\right) z^{3} \\
& +\left(\phi_{4}-\frac{1}{3}\left(18 \zeta_{3} \lambda_{2}+\omega_{1}^{2}\right)\right) z^{4}+\psi_{5} z^{5}+f_{6} z^{6}+f_{7} z^{7}+f_{8} z^{8} \\
g= & \frac{1}{864} \tau^{6}+\frac{1}{72} \tau^{4} \zeta_{3} z+\frac{1}{72} \tau^{2}\left(4 \zeta_{3}^{2}+\tau^{2} \omega_{1}\right) z^{2}+\frac{1}{108}\left(8 \zeta_{3}^{3}+12 \tau^{2} \zeta_{3} \omega_{1}+27 \tau^{4} \lambda_{2}\right) z^{3} \\
& +\frac{1}{36}\left(-3 \tau^{2} \phi_{4}+2\left(4 \zeta_{3}^{2} \omega_{1}+27 \tau^{2} \zeta_{3} \lambda_{2}+\tau^{2} \omega_{1}^{2}\right)\right) z^{4} \\
& +\frac{1}{36}\left(-3\left(4 \zeta_{3} \phi_{4}+\tau^{2} \psi_{5}\right)+4\left(18 \zeta_{3}^{2} \lambda_{2}+2 \zeta_{3} \omega_{1}^{2}+9 \tau^{2} \lambda_{2} \omega_{1}\right)\right) z^{5} \\
& +\left(-\frac{1}{12} \tau^{2} f_{6}-\frac{1}{3}\left(\omega_{1} \phi_{4}+\zeta_{3} \psi_{5}\right)+\frac{2}{27} \omega_{1}^{3}+2 \zeta_{3} \lambda_{2} \omega_{1}+9 \tau^{2} \lambda_{2}^{2}\right) z^{6} \\
& +\left(-\frac{1}{3}\left(18 \lambda_{2} \phi_{4}+\omega_{1} \psi_{5}\right)-\frac{1}{3} \zeta_{3} f_{6}-\frac{1}{12} \tau^{2} f_{7}\right) z^{7}+g_{8} z^{8}+g_{9} z^{9}+g_{10} z^{10}+g_{11} z^{11}+g_{12} z^{12}, \\
\Delta= & z^{8} E_{24+4 n}
\end{aligned}
$$

with an irreducible polynomial

$$
\begin{aligned}
E_{24+4 n}=-\frac{1}{192} \tau^{4} & \left(12 \phi_{4}^{2}+144 \phi_{4} \zeta_{3} \lambda_{2}+432 \zeta_{3}^{2} \lambda_{2}^{2}-12 g_{8} \tau^{2}-4 f_{7} \zeta_{3} \tau^{2}\right. \\
& \left.-72 \lambda_{2} \psi_{5} \tau^{2}-f_{8} \tau^{4}-4 f_{6} \tau^{2} \omega_{1}-432 \lambda_{2}^{2} \tau^{2} \omega_{1}\right)+o(z) .
\end{aligned}
$$

The important difference from the $\mathrm{CY}_{3}$ constructed by Tate's algorithm is that the terms higher than $o\left(z^{4}\right)$ in $f$ or $o\left(z^{6}\right)$ in $g$ contain a term written by only the polynomials that are needed to express $f_{\text {res }}$ and $g_{\text {res }}$. It is $-6 \lambda_{2} \phi_{4} z^{7}$ contained in $o\left(z^{7}\right)$ of $g$. It is the sign that this singularity $G=\mathrm{SU}(8)$ can not be realized in a rational elliptic surface fibration but we need a full-fledged K3 fibration. As a result, the map (4.17) changes the singularity as follows. Mapping $f$ and $g$ to $f^{\prime}$ and $g^{\prime}$, one obtains $\operatorname{ord}\left(\Delta^{\prime}\right)=5$. The $o\left(z^{5}\right)$ and $o\left(z^{6}\right)$ terms of $g^{\prime}$ contain the polynomials

$$
\psi_{5}, f_{6}
$$

which are the coefficients of the terms higher than $o\left(z^{4}\right)$ in $f$ and $o\left(z^{6}\right)$ in $g$. Setting them to zero, we have $\operatorname{ord}\left(\Delta_{\text {res }}\right)=7$. Thus, the singularity is reduced by the map (4.17). ${ }^{10}$

\footnotetext{
${ }^{9}$ This is the $r=0$ case given by setting $\delta=1$ and $\zeta_{4}=0$ in [66].

${ }^{10}$ If we set the term $-6 \lambda_{2} \phi_{4} z^{7}$ of $g$ to zero in advance, i.e., if we set $\lambda_{2}=0$ or $\phi_{4}=0$ in (4.23), the $\mathrm{SU}(8)$ singularity is contained within one $E_{8}$. In these cases, mapping to $f^{\prime}$ and $g^{\prime} \operatorname{gives} \operatorname{ord}\left(\Delta^{\prime}\right)=5$ and setting $\psi_{5}$ and $f_{6}(4.25)$ to zero gives ord $\left(\Delta_{\text {res }}\right)=8$. However, the resulting $\Delta_{\text {res }}$ has a factorized form and additional singularities arise. That is, these cases result in Tate's case described above. Explicitly, $\Delta_{\text {res }}=z^{8} \phi_{4}^{2} \tilde{E}_{8+4 n}$ for $\lambda_{2}=0$ and $\Delta_{\text {res }}=z^{8} \lambda_{2}^{2} \tilde{E}_{16+6 n}$ for $\phi_{4}=0$.
} 


\begin{tabular}{|c|c|}
\hline Representation & Multiplicity \\
\hline$(\mathbf{3}, \mathbf{1}, \mathbf{1}),(\mathbf{1}, \mathbf{3}, \mathbf{1}),(\mathbf{1}, \mathbf{1}, \mathbf{3})$ & $-1(\mathrm{SU}(2) \times \mathrm{SU}(2) \times \mathrm{SU}(2)$ vector $)$ \\
$(\mathbf{1}, \mathbf{1}, \mathbf{1})$ & $6 n+25-4 r$ \\
$(\mathbf{2}, \mathbf{1}, \mathbf{1}),(\mathbf{1}, \mathbf{2}, \mathbf{1}),(\mathbf{1}, \mathbf{1}, \mathbf{2})$ & $2 n+16+2 r$ \\
$(\mathbf{2}, \mathbf{2}, \mathbf{1}),(\mathbf{2}, \mathbf{1}, \mathbf{2}),(\mathbf{1}, \mathbf{2}, \mathbf{2})$ & $n-r$ \\
$\frac{1}{2}(\mathbf{2}, \mathbf{2}, \mathbf{2})$ & $r$ \\
\hline
\end{tabular}

Table 9. The spectrum for $G=\mathrm{SU}(2) \times \mathrm{SU}(2) \times \mathrm{SU}(2)$.

\section{Geometry for $G=\mathrm{SU}(2) \times \mathrm{SU}(2) \times \mathrm{SU}(2)(\mathrm{No} .7)$}

The starting point of our construction is the $\mathrm{CY}_{3}$ for the $G=\mathrm{SU}(2) \times \mathrm{SU}(2) \times \mathrm{SU}(2)$ model. It is No.7 of [51] and its Mordell-Weil lattice is $E(K)=D_{4}^{*} \oplus A_{1}^{*}$. As we claimed in the previous section, there are models that can be written in the Weierstrass form but cannot be written in Tate's form. Therefore, we use the Weierstrass form throughout this paper, but for the $G=\mathrm{SU}(2) \times \mathrm{SU}(2) \times \mathrm{SU}(2)$ model, we start from Tate's form and convert it to the Weierstrass form. ${ }^{11}$ This is because Tate's algorithm is more convenient for realizing product gauge groups.

\subsection{The heterotic spectrum}

In this model, since $H=\mathrm{SO}(8) \times \mathrm{SU}(2)\left(E(K)=D_{4}^{*} \oplus A_{1}^{*}\right)$ is the Cartan type, the heterotic spectrum is determined in a standard way. Let us divide the $12+n$ instantons taking values in $H$ into each factor $\mathrm{SO}(8)$ and $\mathrm{SU}(2)$ such that

$$
\begin{aligned}
& \mathrm{SO}(8): n+8-r, \\
& \mathrm{SU}(2): 4+r .
\end{aligned}
$$

By using the index theorem, we obtain the spectrum as in table 9. The number $n(H)$ of the hypermultiplets is given by

$$
n(H)=(6 n+25-4 r)+(2 n+16+2 r) \cdot 3 \cdot 2+(n-r) \cdot 3 \cdot 2^{2}+r \cdot \frac{1}{2} 2^{3}=30 n+121,
$$

while the number of vector multiplets reads $n(V)=3 \times 3=9$. Therefore, anomaly cancellation condition is satisfied as

$$
n(H)-n(V)=30 n+112 .
$$

\section{$5.2 r=0$ case}

It is necessary to put the $A_{1}$ singularities on three different lines. We first put the $A_{1}$ singularity at $z=0$. From Tate's algorithm (table 8), the orders ord $\left(a_{i}\right)$ are given by $(0,0,1,1,2)$, and hence $a_{i j}$ should satisfy

$$
a_{30}=a_{40}=a_{60}=a_{61}=0 .
$$

\footnotetext{
${ }^{11}$ We can alternatively start from the Weierstrass form for $G=\mathrm{SU}(6)$ and Higgsing it to derive the $\mathrm{SU}(2) \times \mathrm{SU}(2) \times \mathrm{SU}(2)$ model, but the construction by using Tate's algorithm is more straightforward.
} 
Next we put the second $A_{1}$ on another line. This line is taken to be

$$
\tilde{z} \equiv z+h_{n}=0 \text {. }
$$

$z$ is shifted by an order $n$ polynomial $h_{n}\left(z^{\prime}\right)$, so that $\tilde{z}$ is homogeneous under the transformation (4.4). To realize the $A_{1}$ singularity at $\tilde{z}=0$, we re-expand $a_{i}$ in terms of $\tilde{z}$ as

$$
a_{i}(z)=a_{i}\left(\tilde{z}-h_{n}\right)=\sum_{j=0}^{2 i} \tilde{a}_{i j} \tilde{z}^{j}
$$

and impose on $\tilde{a}_{i j}$ the same conditions as in (5.4),

$$
\tilde{a}_{30}=\tilde{a}_{40}=\tilde{a}_{60}=\tilde{a}_{61}=0 .
$$

Finally, we place the third $A_{1}$ at

$$
\hat{z} \equiv z+h_{n}^{\prime}=0
$$

and re-expand $a_{i}$ as

$$
a_{i}(z)=a_{i}\left(\hat{z}-h_{n}^{\prime}\right)=\sum_{j=0}^{2 i} \hat{a}_{i j} \hat{z}^{j}
$$

and impose

$$
\hat{a}_{30}=\hat{a}_{40}=\hat{a}_{60}=\hat{a}_{61}=0 .
$$

The resulting form of $a_{i}$ 's are given as follows:

$$
\begin{aligned}
& a_{1}(z)=a_{10}+a_{11} z+a_{12} z^{2}, \\
& a_{2}(z)=a_{20}+a_{21} z+a_{22} z^{2}+a_{23} z^{3}+a_{24} z^{4}, \\
& a_{3}(z)=z\left(z+h_{n}\right)\left(z+h_{n}^{\prime}\right)\left\{\left(a_{33}+\cdots\right)+\left(a_{34}+\cdots\right) z+\cdots+\left(a_{36}+\cdots\right) z^{3}\right\}, \\
& a_{4}(z)=z\left(z+h_{n}\right)\left(z+h_{n}^{\prime}\right)\left\{\left(a_{43}+\cdots\right)+\left(a_{44}+\cdots\right) z+\cdots+\left(a_{48}+\cdots\right) z^{5}\right\}, \\
& a_{6}(z)=z^{2}\left(z+h_{n}\right)^{2}\left(z+h_{n}^{\prime}\right)^{2}\left\{\left(a_{66}+\cdots\right)+\left(a_{67}+\cdots\right) z+\cdots+\left(a_{6,12}+\cdots\right) z^{6}\right\},
\end{aligned}
$$

where $(\cdots)$ are written by $a_{i j}, h_{n}$ and $h_{n}^{\prime}$. To translate this Tate's form into the Weierstrass form, we calculate $b_{i}$ (4.10). Although $b_{i}$ have complicated dependence of $a_{i j}, h_{n}$ and $h_{n}^{\prime}$, one can redefine $a_{i j}$ so that they are arranged to have simple forms:

$$
\begin{aligned}
& b_{2}=A_{20}+A_{21} z+\cdots+A_{24} z^{4}, \\
& b_{4}=z\left(z+h_{n}\right)\left(z+h_{n}^{\prime}\right)\left\{A_{43}+A_{44} z+\cdots+A_{48} z^{5}\right\}, \\
& b_{6}=z^{2}\left(z+h_{n}\right)^{2}\left(z+h_{n}^{\prime}\right)^{2}\left\{A_{66}+A_{67} z+\cdots+A_{6,12} z^{6}\right\} .
\end{aligned}
$$

Substituting them into (4.11), we obtain $f$ and $g$ in terms of $A_{i j}$. To arrange so that the middle polynomials $f_{8}$ and $g_{12}$ have simple form, we further redefine as

$$
\tilde{A}_{44} \equiv A_{44}-\frac{1}{3} A_{22}^{2}, \quad \tilde{A}_{66} \equiv A_{66}+\frac{1}{27}\left(2 A_{22}^{3}-9 A_{22} A_{44}\right) .
$$

Similarly, we redefine $A_{6 j}$ with $j=7,8,9,10$ by subtracting $A_{22}$ dependent terms:

$$
\tilde{A}_{6 j} \equiv A_{6 j}-\frac{1}{3} A_{22} A_{4 j-2} \quad(j=7,8,9,10) .
$$


Moreover, we found that $A_{23}$ and $A_{24}$ can be discarded from $f$ and $g$ without any effect on the singularity structure. As a result, we obtain the following form:

$$
\begin{aligned}
& f=-\frac{1}{3} A_{20}^{2}+\left(-\frac{2}{3} A_{20} A_{21}+A_{43} \sigma_{2}\right) z+\frac{1}{3}\left(-A_{21}^{2}-2 A_{20} A_{22}+3 A_{43} \sigma_{1}+\left(A_{22}^{2}+3 \tilde{A}_{44}\right) \sigma_{2}\right) z^{2} \\
& +\frac{1}{3}\left(-2 A_{21} A_{22}+3 A_{43}+\left(A_{22}^{2}+3 \tilde{A}_{44}\right) \sigma_{1}+3 A_{45} \sigma_{2}\right) z^{3} \\
& +\left(\tilde{A}_{44}+A_{45} \sigma_{1}+A_{46} \sigma_{2}\right) z^{4}+\left(A_{45}+A_{46} \sigma_{1}+A_{47} \sigma_{2}\right) z^{5} \\
& +\left(A_{46}+A_{47} \sigma_{1}+A_{48} \sigma_{2}\right) z^{6}+\left(A_{47}+A_{48} \sigma_{1}\right) z^{7}+A_{48} z^{8} \\
& g=\frac{2}{27} A_{20}^{3}+\frac{1}{9}\left(2 A_{20}^{2} A_{21}-3 A_{20} A_{43} \sigma_{2}\right) z \\
& +\frac{1}{27}\left(6\left(A_{20} A_{21}^{2}+A_{20}^{2} A_{22}\right)-9 A_{20} A_{43} \sigma_{1}\right. \\
& \left.-3\left(3 A_{21} A_{43}+A_{20} A_{22}^{2}+3 A_{20} \tilde{A}_{44}\right) \sigma_{2}+\left(A_{22}^{3}+9 A_{22} \tilde{A}_{44}+27 \tilde{A}_{66}\right) \sigma_{2}^{2}\right) z^{2} \\
& +\frac{1}{27}\left(2 A_{21}^{3}+12 A_{20} A_{21} A_{22}-9 A_{20} A_{43}-3\left(3 A_{21} A_{43}+A_{20} A_{22}^{2}+3 A_{20} \tilde{A}_{44}\right) \sigma_{1}\right. \\
& -3\left(3 A_{22} A_{43}+A_{21} A_{22}^{2}+3 A_{21} \tilde{A}_{44}+3 A_{20} A_{45}\right) \sigma_{2} \\
& \left.+2\left(A_{22}^{3}+9 A_{22} \tilde{A}_{44}+27 \tilde{A}_{66}\right) \sigma_{1} \sigma_{2}+9\left(A_{22} A_{45}+3 \tilde{A}_{67}\right) \sigma_{2}^{2}\right) z^{3} \\
& +\frac{1}{27}\left(3\left(2 A_{21}^{2} A_{22}+A_{20} A_{22}^{2}-3 A_{21} A_{43}-3 A_{20} \tilde{A}_{44}\right)\right. \\
& -3\left(3 A_{22} A_{43}+A_{21} A_{22}^{2}+3 A_{21} \tilde{A}_{44}+3 A_{20} A_{45}\right) \sigma_{1}+\left(A_{22}^{3}+9 A_{22} \tilde{A}_{44}+27 \tilde{A}_{66}\right) \sigma_{1}^{2} \\
& -\left(A_{22}^{3}-9 A_{22} \tilde{A}_{44}+9 A_{21} A_{45}+9 A_{20} A_{46}-54 \tilde{A}_{66}\right) \sigma_{2} \\
& \left.+18\left(A_{22} A_{45}+3 \tilde{A}_{67}\right) \sigma_{1} \sigma_{2}+9\left(A_{22} A_{46}+3 \tilde{A}_{68}\right) \sigma_{2}^{2}\right) z^{4} \\
& +\frac{1}{27}\left(3\left(A_{21} A_{22}^{2}-3 A_{22} A_{43}-3 A_{21} \tilde{A}_{44}-3 A_{20} A_{45}\right)\right. \\
& -\left(A_{22}^{3}-9 A_{22} \tilde{A}_{44}+9 A_{21} A_{45}+9 A_{20} A_{46}-54 \tilde{A}_{66}\right) \sigma_{1}+9\left(A_{22} A_{45}+3 \tilde{A}_{67}\right) \sigma_{1}^{2} \\
& +9\left(6 \tilde{A}_{67}+A_{22} A_{45}-A_{21} A_{46}-A_{20} A_{47}\right) \sigma_{2} \\
& \left.+18\left(A_{22} A_{46}+3 \tilde{A}_{68}\right) \sigma_{1} \sigma_{2}+9\left(A_{22} A_{47}+3 \tilde{A}_{69}\right) \sigma_{2}^{2}\right) z^{5} \\
& +\frac{1}{3}\left(3 \tilde{A}_{66}-A_{21} A_{45}-A_{20} A_{46}+\left(6 \tilde{A}_{67}+A_{22} A_{45}-A_{21} A_{46}-A_{20} A_{47}\right) \sigma_{1}+\left(A_{22} A_{46}+3 \tilde{A}_{68}\right) \sigma_{1}^{2}\right. \\
& +\left(6 \tilde{A}_{68}+A_{22} A_{46}-A_{21} A_{47}-A_{20} A_{48}\right) \sigma_{2}+2\left(A_{22} A_{47}+3 \tilde{A}_{69}\right) \sigma_{1} \sigma_{2} \\
& \left.+\left(A_{22} A_{48}+3 \tilde{A}_{6,10}\right) \sigma_{2}^{2}\right) z^{6} \\
& +\frac{1}{3}\left(3 \tilde{A}_{67}-A_{21} A_{46}-A_{20} A_{47}+\left(6 \tilde{A}_{68}+A_{22} A_{46}-A_{21} A_{47}-A_{20} A_{48}\right) \sigma_{1}+\left(A_{22} A_{47}+3 \tilde{A}_{69}\right) \sigma_{1}^{2}\right. \\
& \left.+\left(6 \tilde{A}_{69}+A_{22} A_{47}-A_{21} A_{48}\right) \sigma_{2}+2\left(A_{22} A_{48}+3 \tilde{A}_{6,10}\right) \sigma_{1} \sigma_{2}+3 A_{6,11} \sigma_{2}^{2}\right) z^{7} \\
& +\frac{1}{3}\left(3 \tilde{A}_{68}-A_{21} A_{47}-A_{20} A_{48}+\left(6 \tilde{A}_{69}+A_{22} A_{47}-A_{21} A_{48}\right) \sigma_{1}+\left(A_{22} A_{48}+3 \tilde{A}_{6,10}\right) \sigma_{1}^{2}\right. \\
& \left.+\left(6 \tilde{A}_{6,10}+A_{22} A_{48}\right) \sigma_{2}+6 A_{6,11} \sigma_{1} \sigma_{2}+3 A_{6,12} \sigma_{2}^{2}\right) z^{8} \\
& +\frac{1}{3}\left(3 \tilde{A}_{69}-A_{21} A_{48}+\left(6 \tilde{A}_{6,10}+A_{22} A_{48}\right) \sigma_{1}+3 A_{6,11}\left(\sigma_{1}^{2}+2 \sigma_{2}\right)+6 A_{6,12} \sigma_{1} \sigma_{2}\right) z^{9} \\
& +\left(\tilde{A}_{6,10}+2 A_{6,11} \sigma_{1}+A_{6,12}\left(\sigma_{1}^{2}+2 \sigma_{2}\right)\right) z^{10}+\left(A_{6,11}+2 A_{6,12} \sigma_{1}\right) z^{11}+A_{6,12} z^{12} .
\end{aligned}
$$


Here the dependence on $h_{n}$ and $h_{n}^{\prime}$ is included only through the symmetric polynomials $\sigma_{1}, \sigma_{2}$ as it should be because the lines of $A_{1}$ singularity can be replaced with each other:

$$
\begin{aligned}
& \sigma_{1}=h_{n}+h_{n}^{\prime}, \\
& \sigma_{2}=h_{n} h_{n}^{\prime} .
\end{aligned}
$$

The discriminant has the form

$$
\Delta=z^{2}\left(z+h_{n}\right)^{2}\left(z+h_{n}^{\prime}\right)^{2} D_{6 n+24}
$$

where $D_{6 n+24}$ is an irreducible polynomial written by $A_{i j}, h_{n}$ and $h_{n}^{\prime}$. The leading expansion is given by

$$
\begin{aligned}
D_{6 n+24}=\frac{1}{27} A_{20}^{2}\left(4 A_{20} A_{22}^{3}-27 A_{43}^{2}+36 A_{20} A_{22} \tilde{A}_{44}+108 A_{20} \tilde{A}_{66}\right) \\
+\frac{2}{9}\left(A _ { 2 0 } \left\{-3 A_{43}\left(A_{20} A_{22}^{2}+3 A_{21} A_{43}+3 A_{20} \tilde{A}_{44}\right)\right.\right. \\
\left.\quad+2 A_{20} A_{21}\left(A_{22}^{3}+9 A_{22} \tilde{A}_{44}+27 \tilde{A}_{66}\right)+6 A_{20}^{2} A_{22} A_{45}+18 A_{20}^{2} \tilde{A}_{67}\right\} \\
\left.\quad-3 A_{43}\left\{-6 A_{43}^{2}+A_{20}\left(A_{22}^{3}+9 A_{22} \tilde{A}_{44}+27 \tilde{A}_{66}\right)\right\} \sigma_{2}\right) z+o\left(z^{2}\right) .
\end{aligned}
$$

We map these $f$ and $g$ to $f^{\prime}$ and $g^{\prime}$. Then $f^{\prime}$ and $g^{\prime}$ contain the polynomials

$$
\begin{aligned}
& A_{45}, A_{46}, A_{47}, A_{48}, \\
& \tilde{A}_{67}, \tilde{A}_{68}, \tilde{A}_{69}, \tilde{A}_{6,10},
\end{aligned}
$$

which constitute the coefficients of the terms higher than $o\left(z^{4}\right)$ in $f$ and $o\left(z^{6}\right)$ in $g$. Setting them to zero, one obtains a RES-fibered geometry. One can see from the explicit forms of $f_{\text {res }}, g_{\text {res }}$ and $\Delta_{\text {res }}$ that the singularity remains to be $G=\mathrm{SU}(2) \times \mathrm{SU}(2) \times \mathrm{SU}(2)$ (see (5.22), (5.23) and (5.24) below).

From this geometry, let us extract the matter spectrum. Singlets correspond to the moduli space $\mathcal{M}(H)$ of the gauge bundle $H$, which is identified with the complex moduli of the geometry, except for the middle polynomials $f_{8}$ and $g_{12}$ belonging to the geometric moduli of the heterotic K3. The number of singlets, therefore, is the number of degrees of independent polynomials contained in $f_{\text {res }}$ and $g_{\text {res }}$ except $f_{8}$ and $g_{12}$. Charged matters are localized at codimension two loci of singularities on the base space. Suppose the generic codimension one singularity is $G$ and it enhances to $G^{\prime}$ at a codimension two locus. Then there is a matter in a representation corresponding to the off diagonal part of $G \subset G^{\prime}{ }^{12}$

In the present case, $f_{\text {res }}$ and $g_{\text {res }}$ contain the following six independent polynomials (apart from $\tilde{A}_{44}$ and $\tilde{A}_{66}$, which are not counted because they correspond to the middle

\footnotetext{
${ }^{12}$ It is justified in the type IIB picture by using the notion of string junctions [73].
} 
polynomials $f_{8}$ and $\left.g_{12}\right)$ :

\begin{tabular}{|c|c|}
\hline Polynomial & Degree \\
\hline$h_{n}$ & $n$ \\
$h_{n}^{\prime}$ & $n$ \\
$A_{20}$ & $4+2 n$ \\
$A_{21}$ & $4+n$ \\
$A_{22}$ & 4 \\
$A_{43}$ & $8+n$ \\
\hline
\end{tabular}

The degree of each polynomial is given by (4.8). The number $n\left(H_{0}\right)$ of the singlet (neutral hypermultiplet) is thus calculated as

$$
n\left(H_{0}\right)=n+n+(4+2 n)+(4+n)+4+(8+n)+6-1=6 n+25,
$$

where $(-1)$ is the overall rescaling.

To see where and how the singularity enhances, we expand $f_{\text {res }}, g_{\text {res }}$ and $\Delta_{\text {res }}$ near each of the three lines $z=0, \tilde{z}=0$ and $\hat{z}=0$. The results are

$$
\begin{aligned}
f_{\text {res }} & =-\frac{1}{3} A_{20}^{2}+o(z) \\
& =-\frac{1}{3} \tilde{A}_{20}^{2}+o(\tilde{z}) \\
& =-\frac{1}{3} \hat{A}_{20}^{2}+o(\hat{z}), \\
g_{\text {res }} & =\frac{2}{27} A_{20}^{3}+(\cdots) A_{20} z+o\left(z^{2}\right) \\
& =\frac{2}{27} \tilde{A}_{20}^{3}+(\cdots) \tilde{A}_{20} \tilde{z}+o\left(\tilde{z}^{2}\right) \\
& =\frac{2}{27} \hat{A}_{20}^{3}+(\cdots) \hat{A}_{20} \hat{z}+o\left(\hat{z}^{2}\right), \\
\Delta_{\text {res }} & =A_{20}^{2} h_{n}^{2} h_{n}^{\prime 2} P_{16+2 n} z^{2}+(\cdots) h_{n} h_{n}^{\prime} z^{3}+o\left(z^{4}\right) \\
& =\tilde{A}_{20}^{2} h_{n}^{2}\left(h_{n}-h_{n}^{\prime}\right)^{2} \tilde{P}_{16+2 n} \tilde{z}^{2}+(\cdots) h_{n}\left(h_{n}-h_{n}^{\prime}\right) \tilde{z}^{3}+o\left(\tilde{z}^{4}\right) \\
& =\hat{A}_{20}^{2} h_{n}^{\prime 2}\left(h_{n}-h_{n}^{\prime}\right)^{2} \hat{P}_{16+2 n} \hat{z}^{2}+(\cdots) h_{n}^{\prime}\left(h_{n}-h_{n}^{\prime}\right) \hat{z}^{3}+o\left(\hat{z}^{4}\right) .
\end{aligned}
$$

Here

$$
\begin{aligned}
& \tilde{A}_{20}=A_{20}-A_{21} h_{n}+A_{22} h_{n}^{2}, \\
& \hat{A}_{20}=A_{20}-A_{21} h_{n}^{\prime}+A_{22} h_{n}^{\prime 2}
\end{aligned}
$$

and $P_{16+2 n}, \tilde{P}_{16+2 n}$ and $\hat{P}_{16+2 n}$ are degree $16+2 n$ irreducible polynomials. We represented as $(\cdots)$ the factors irrelevant to the symmetry enhancement for simplicity. From these expansions, one can read the loci and types of enhancements, and then one finds what kind 
of representations of matters appear at those points. The result is given by

\begin{tabular}{|l|c|ccc|c|c|}
\hline \multicolumn{1}{|c|}{ Zero } & Degree & ord $\left(f_{\text {res }}\right)$ & ord $\left(g_{\text {res }}\right)$ ord $\left(\Delta_{\text {res }}\right)$ & Enhancement & Matter \\
\hline$A_{20}, \tilde{A}_{20}, \hat{A}_{20}$ & $4+2 n$ & 1 & 2 & 3 & $A_{1} \rightarrow A_{1}\left(I_{2} \rightarrow I I I\right)$ & none \\
\hline$P_{16+2 n}$ & $16+2 n$ & 0 & 0 & 3 & $A_{1} \rightarrow A_{2}$ & $(\mathbf{2}, \mathbf{1}, \mathbf{1})$ \\
$\tilde{P}_{16+2 n}$ & $16+2 n$ & 0 & 0 & 3 & $A_{1} \rightarrow A_{2}$ & $(\mathbf{1}, \mathbf{2}, \mathbf{1})$ \\
$\hat{P}_{16+2 n}$ & $16+2 n$ & 0 & 0 & 3 & $A_{1} \rightarrow A_{2}$ & $(\mathbf{1}, \mathbf{1}, \mathbf{2})$ \\
\hline$h_{n}$ & $n$ & 0 & 0 & 4 & $A_{1} \oplus A_{1} \rightarrow A_{3}$ & $(\mathbf{2}, \mathbf{2}, \mathbf{1})$ \\
$h_{n}^{\prime}$ & $n$ & 0 & 0 & 4 & $A_{1} \oplus A_{1} \rightarrow A_{3}$ & $(\mathbf{2}, \mathbf{1}, \mathbf{2})$ \\
$h_{n}-h_{n}^{\prime}$ & $n$ & 0 & 0 & 4 & $A_{1} \oplus A_{1} \rightarrow A_{3}$ & $(\mathbf{1}, \mathbf{2}, \mathbf{2})$ \\
\hline
\end{tabular}

This table is obtained as follows. We first pay attention on the leading term of $\Delta_{\text {res }}$ in (5.24). The coefficient is a product of several factors. If one of them vanishes, the order of $\Delta_{\text {res }}$ enhances. The list of such factors are shown in the first column and its degree is shown in the second column. When a factor vanishes, not only the order of $\Delta_{\text {res }}$ but also the orders of $f_{\text {res }}$ and $g_{\text {res }}$ enhance in general. To what extent they will enhance can be read from (5.22), (5.23) and (5.24) and given in the next three columns. The resulting enhancement can be read from table 7 and is shown in the next column. The associated matter representation is given in the last column, where the three entries correspond to the three $A_{1}$ at $z=0, \tilde{z}=0$ and $\hat{z}=0$ in this order. As we can see, unresolvable singularity does not appear anywhere.

Let us give some comments. Consider $A_{20}=0$ in the first row. In this case, the fiber degeneracy is enhanced from $I_{2}$ to $I I I$, but the singularity does not change and remains to be $A_{1}$. Therefore, there exists no matter at these points. The same is true for $\tilde{A}_{20}=0$ and $\hat{A}_{20}=0$. When $h_{n}=0$ (the fifth row), the orders of expansions around $z=0$ and $\tilde{z}=0$ simultaneously enhance to $A_{3}$, while the orders of $\hat{z}$ do not change. This reflects the fact that the two lines $z=0$ and $\tilde{z}=0$ intersect at $h_{n}=0$, giving rise to matter in the bi-fundamental representation $(\mathbf{2}, \mathbf{2}, \mathbf{1})$. A similar thing is true for $h_{n}^{\prime}=0$ and $h_{n}-h_{n}^{\prime}=0$.

Together with the singlets (5.21), the total spectrum is given by

$$
\begin{aligned}
6 n+25 & :(\mathbf{1}, \mathbf{1}, \mathbf{1}) \\
2 n+16 & :(\mathbf{2}, \mathbf{1}, \mathbf{1}),(\mathbf{1}, \mathbf{2}, \mathbf{1}),(\mathbf{1}, \mathbf{1}, \mathbf{2}) \\
n & :(\mathbf{2}, \mathbf{2}, \mathbf{1}),(\mathbf{2}, \mathbf{1}, \mathbf{2}),(\mathbf{1}, \mathbf{2}, \mathbf{2})
\end{aligned}
$$

This F-theoretic spectrum coincides with the heterotic spectrum (table 9) with $r=0$.

\section{$5.3 r \neq 0$ case}

We next construct a $\mathrm{CY}_{3}$ for general distributions of instantons and then map it to a RESfibered geometry. As seen in the previous section, Tate's algorithm gives the $r=0$ case only. This means that the $r \neq 0$ case is not obtained by Tate's algorithm and we have to use the Weierstrass form. So we start from the Weierstrass form (5.15) for the $r=0$ case and deform the equation appropriately. In order to know how to deform it, we use one particular 
information from the heterotic spectrum in table 9 as an input. The only input data is the appearance of $r$ half-hypermultiplets in the tri-fundamental representation $\frac{1}{2}(\mathbf{2}, \mathbf{2}, \mathbf{2})$. We determine the $\mathrm{CY}_{3}$ so that they are contained. As we will show below, it turns out that this requirement uniquely determines the $\mathrm{CY}_{3}$. After mapping to a RES-fibered geometry, we derive the full spectrum. The resulting F-theoretic full spectrum perfectly matches with the heterotic full spectrum (not only the part we used as an input).

It is expected that tri-fundamental representation is localized at a triple intersection point of three lines of $A_{1}$ singularity. For a triple intersection point to exist, $z=0, \tilde{z}=0$ and $\hat{z}=0$ need to share a common solution, that is,

$$
z=0, \quad h_{n}=h_{n}^{\prime}=0 .
$$

Therefore, $h_{n}$ and $h_{n}^{\prime}$ have a common factor. Writing this factor as $t_{r}$, we have

$$
\begin{aligned}
& h_{n}=h_{n-r} t_{r}, \\
& h_{n}^{\prime}=h_{n-r}^{\prime} t_{r} .
\end{aligned}
$$

Three lines intersect at $r$ points satisfying $z=0$ and $t_{r}\left(z^{\prime}\right)=0$.

The next question is how much the gauge symmetry is enhanced where a trifundamental appears. As we argue below, it should be

$$
\mathrm{SU}(2) \times \mathrm{SU}(2) \times \mathrm{SU}(2) \rightarrow \mathrm{SO}(8) .
$$

Let us first look at the branching of $\mathrm{SO}(8) \supset \mathrm{SU}(2) \times \mathrm{SU}(2) \times \mathrm{SU}(2)$ :

$$
\mathbf{2 8}=(\mathbf{3}, \mathbf{1}, \mathbf{1}) \oplus(\mathbf{1}, \mathbf{3}, \mathbf{1}) \oplus(\mathbf{1}, \mathbf{1}, \mathbf{3}) \oplus \mathbf{3}(\mathbf{1}, \mathbf{1}, \mathbf{1}) \oplus \mathbf{2}(\mathbf{2}, \mathbf{2}, \mathbf{2}) .
$$

The tri-fundamental is contained in the off-diagonal elements. Moreover, there is a maximal embedding $\mathrm{SO}(8) \supset \mathrm{SU}(2) \times \mathrm{SU}(2) \times \mathrm{SU}(2) \times \mathrm{SU}(2)$, whose branching is given by

$$
28=(3,1,1,1) \oplus(1,3,1,1) \oplus(1,1,3,1) \oplus(1,1,1,3) \oplus(2,2,2,2) .
$$

In the branching of the maximal embedding $G \supset G^{\prime} \times H^{\prime}$ with $H^{\prime}=\mathrm{SU}(2)$, the representation of $G^{\prime}$ which is combined with 2 of $H^{\prime}$ is a pseudo-real representation. In the present case (5.32), the tri-fundamental representation of $G^{\prime}=\mathrm{SU}(2) \times \mathrm{SU}(2) \times \mathrm{SU}(2)$ is pseudo-real, forming a half-hypermultiplet $\frac{1}{2}(\mathbf{2}, \mathbf{2}, \mathbf{2}) .{ }^{13}$

In order that the singularity is enhanced to $\mathrm{SO}(8)$ at $t_{r}=0$, we have to tune the geometry so that $(\operatorname{ord}(f), \operatorname{ord}(g), \operatorname{ord}(\Delta))=(2,3,6)$ at $t_{r}=0$. Let us first notice that $\sigma_{1}=\sigma_{2}=0$ at $t_{r}=0$. One can then easily see from (5.15) that $(\operatorname{ord}(f), \operatorname{ord}(g))=(2,3)$ at $t_{r}=0$ if and only if $A_{20}$ is factorized as (recall that the degree of $A_{20}$ is given by (4.8))

$$
A_{20}=p_{4+2 n-r} t_{r}
$$

\footnotetext{
${ }^{13}$ We list below the other examples of arising half-hypermultiplets given in [70]. Why half-hypermultiplets appear in these cases has been explained from string junctions' point of view [73].

\begin{tabular}{|llll|}
\hline Enhancement & Maximal embedding & Branching & Matter \\
\hline$E_{7} \rightarrow E_{8}$ & $E_{8} \supset E_{7} \times \mathrm{SU}(2)$ & $\mathbf{2 4 8}=(\mathbf{1 3 3}, \mathbf{1}) \oplus(\mathbf{1}, \mathbf{3}) \oplus(\mathbf{5 6}, \mathbf{2})$ & $\frac{1}{2} \mathbf{5 6}$ \\
$\mathrm{SO}(12) \rightarrow E_{7}$ & $E_{7} \supset \mathrm{SO}(12) \times \mathrm{SU}(2)$ & $\mathbf{1 3 3}=(\mathbf{6 6}, \mathbf{1}) \oplus(\mathbf{1}, \mathbf{3}) \oplus(\mathbf{3 2}, \mathbf{2})$ & $\frac{1}{2} \mathbf{3 2}$ \\
\hline
\end{tabular}
}


Calculating $\Delta$ explicitly, however, we find that it is over-enhanced to $\operatorname{ord}(\Delta)=8$ at $t_{r}=0$. We have to suppress it to $\operatorname{ord}(\Delta)=6$ while keeping $(\operatorname{ord}(f), \operatorname{ord}(g))=(2,3)$. To absorb the excessive factors of $t_{r}$ in $\Delta$, we have to factorize $t_{r}^{-1}$ for at least one polynomial as

$$
A=\frac{A^{\text {new }}}{t_{r}} \text {. }
$$

But as a side effect, this replacement would cause poles $t_{r}^{-n}$ in several terms of $f$ and $g$. To keep the orders of $f$ and $g$ fixed, it should be accompanied with the factorization of other polynomial

$$
B=B^{\text {new }} t_{r}
$$

Take a term in $f$ or $g$. If $A$ is contained in the coefficient of that term as a product $A B$, we can "interchange the factor $t_{r}$ " to absorb the pole as [66]

$$
A B=\frac{A^{\text {new }}}{t_{r}} B=A^{\text {new }} B^{\text {new }} .
$$

On the other hand, if $A$ is contained as $A^{n}$ for some $n$, the pole cannot be cancelled.

Among the polynomials contained in the $\mathrm{CY}_{3}$ for $r=0$ case (5.15), only those listed in (5.20) can change the singularity type near $z=0 . h_{n}, h_{n}^{\prime}$ and $A_{20}$ are already factorized. The remainings are $A_{21}, A_{22}$ and $A_{43}$. Among them, $A_{22}$ is the one that should be divided by $t_{r}$. The reason is as follows. Substituting (5.29) and (5.33) into (5.15), we obtain

$$
\begin{aligned}
f= & -\frac{1}{3} p_{4+2 n-r}^{2} t_{r}^{2}+\left(-\frac{2}{3} p_{4+2 n-r} A_{21} t_{r}+A_{43} \hat{\sigma}_{2} t_{r}^{2}\right) z \\
& +\frac{1}{3}\left(-A_{21}^{2}-2 A_{22} p_{4+2 n-r} t_{r}+3 A_{43} t_{r} \hat{\sigma}_{1}+\left(A_{22}^{2}+3 \tilde{A}_{44}\right) \hat{\sigma}_{2} t_{r}^{2}\right) z^{2} \\
& +\frac{1}{3}\left(-2 A_{21} A_{22}+3 A_{43}+\left(A_{22}^{2}+3 \tilde{A}_{44}\right) \hat{\sigma}_{1} t_{r}+3 A_{45} \hat{\sigma}_{2} t_{r}^{2}\right) z^{3} \\
& +\left(\tilde{A}_{44}+A_{45} \hat{\sigma}_{1} t_{r}+A_{46} \hat{\sigma}_{2} t_{r}^{2}\right) z^{4}+o\left(z^{5}\right),
\end{aligned}
$$

where we defined the reduced symmetric polynomials as

$$
\begin{aligned}
& \hat{\sigma}_{1}=h_{n-r}+h_{n-r}^{\prime}, \\
& \hat{\sigma}_{2}=h_{n-r} h_{n-r}^{\prime} .
\end{aligned}
$$

Let us look at the $o\left(z^{2}\right)$ term. It contains $A_{21}$ as $-\frac{1}{3} A_{21}^{2}$. This term cannot be eliminated by any redefinitions of other polynomials in the $o\left(z^{2}\right)$ term. Since it has the form $A^{n}$, the interchange of $t_{r}$ (5.36) does not work. We therefore cannot remove the negative power $t_{r}^{-2}$ that would arise if we imposed $A_{21}=A_{21}^{\text {new }} / t_{r}$. The same argument holds for the polynomial $A_{43}$ contained in the $o\left(z^{3}\right)$ term. As a result, we are forced to set $A_{22}=A_{22}^{\text {new }} / t_{r}$. In this case, the procedure of interchanging $t_{r}$ does work. To apply it, we have to do in advance some redefinitions of polynomials so that $A_{22}$ appears in $f$ and $g$ as the form $A_{22} \times B$.

One can check from (5.15) the following fact: if $A_{22}=A_{22}^{\text {new }} / t_{r}$ was imposed, negative power terms would arise only at $o\left(z^{3}\right)$ in $f$ and $o\left(z^{4}\right), o\left(z^{5}\right)$ in $g$. Consider the $o\left(z^{5}\right)$ terms in $g$ first. The negative powers of $t_{r}$ arise from

$$
g=\cdots+\frac{1}{3}\left[-A_{22}\left(A_{43}-\frac{1}{3} A_{22} A_{21}+\frac{1}{9} A_{22}^{2} \hat{\sigma}_{1} t_{r}\right)+\cdots\right] z^{5}+\cdots
$$


In order to have the form $A_{22} \times B$, we should redefine

$$
A_{43}^{\prime} \equiv A_{43}-\frac{1}{3} A_{22} A_{21}+\frac{1}{9} A_{22}^{2} \hat{\sigma}_{1} t_{r}
$$

Next, we rewrite the $o\left(z^{3}\right)$ terms in $f$ by using $A_{43}^{\prime}$ instead of $A_{43}$. The negative powers of $t_{r}$ come from

$$
f=\cdots+\frac{1}{3}\left[-A_{22}\left(A_{21}-\frac{2}{3} A_{22} \hat{\sigma}_{1} t_{r}\right)+\cdots\right] z^{3}+\cdots .
$$

It requires the redefinition

$$
A_{21}^{\prime} \equiv A_{21}-\frac{2}{3} A_{22} \hat{\sigma}_{1} t_{r}
$$

Now we are ready to perform the interchange of $t_{r}$. It is given by

$$
\begin{aligned}
& A_{22}=\frac{q_{4+r}}{t_{r}}, \\
& A_{43}^{\prime}=j_{8+n-r} t_{r}, \\
& A_{21}^{\prime}=k_{4+n-r} t_{r} .
\end{aligned}
$$

The remaining sources of negative powers of $t_{r}$ are contained in the $o\left(z^{4}\right)$ terms in $g$. Substituting all the factorizations above into (5.15), one finds that the worst terms are proportional to $t_{r}^{-1}$ and given by

$$
g=\cdots+\frac{1}{27}\left[\frac{1}{t_{r}} 3 q_{4+r}^{2}\left\{p_{4+2 n-r}-\frac{1}{3} q_{4+r} \hat{\sigma}_{2}\right\}+(\text { regular terms })\right] z^{4}+\cdots .
$$

The negative power is cancelled if and only if $\{\cdots\}$ is proportional to $t_{r}$, i.e.,

$$
p_{4+2 n-r}=s_{4+2 n-2 r} t_{r}+\frac{1}{3} q_{4+r} \hat{\sigma}_{2} .
$$

One can show that all these factorizations and redefinitions not merely cancel the $t_{r}^{-n}$ terms, ${ }^{14}$ but indeed realize just the desired order $(\operatorname{ord}(f), \operatorname{ord}(g), \operatorname{ord}(\Delta))=(2,3,6)$ at $t_{r}=0$. Also, $\Delta$ has the form

$$
\Delta=z^{2}\left(z+h_{n-r} t_{r}\right)^{2}\left(z+h_{n-r}^{\prime} t_{r}\right)^{2} D_{6 n+24}
$$

where $D_{6 n+24}$ is the same polynomial as given in (5.17) and is still irreducible after the above factorizations and redefinitions. To summarize, we obtained a $\mathrm{CY}_{3}$ with enhancement $\mathrm{SU}(2) \times \mathrm{SU}(2) \times \mathrm{SU}(2) \rightarrow \mathrm{SO}(8)$ at $r$ points by imposing on (5.15) the factorizations (5.29), (5.33), (5.43), (5.45) with the redefinitions (5.40), (5.42).

After mapping $f$ and $g$ to $f^{\prime}$ and $g^{\prime}$ and then setting the polynomials (5.19) to zero, one obtains a RES-fibered geometry with $G=\mathrm{SU}(2) \times \mathrm{SU}(2) \times \mathrm{SU}(2)$. The explicit forms of $f_{\text {res }}, g_{\text {res }}$ and $\Delta_{\text {res }}$ are given in appendix C.1.

\footnotetext{
${ }^{14}$ Via the replacement $A_{22}=\frac{q_{4+r}}{t_{r}}(5.43)$, no pole arises in higher order terms. It is because we subtracted the $A_{22}$ dependent terms in advance in (5.14).
} 
We have derived a RES-fibered geometry whose spectrum contains $r$ half-hypermultiplet $\frac{1}{2}(\mathbf{2}, \mathbf{2}, \mathbf{2})$. However, it is not obvious whether the geometry reproduces the other part of the spectrum in table 9 . We will show this is the case. The number of singlets is determined by the independent polynomials. They are the following 7 polynomials defined above:

\begin{tabular}{|c|c|}
\hline Polynomial & Degree \\
\hline$t_{r}$ & $r$ \\
$h_{n-r}$ & $n-r$ \\
$h_{n-r}^{\prime}$ & $n-r$ \\
$s_{4+2 n-2 r}$ & $4+2 n-2 r$ \\
$k_{4+n-r}$ & $4+n-r$ \\
$q_{4+r}$ & $4+r$ \\
$j_{8+n-r}$ & $8+n-r$ \\
\hline
\end{tabular}

The number of the singlets are given by

$$
\begin{aligned}
n\left(H_{0}\right) & =r+(n-r)+(n-r)+(4+2 n-2 r)+(4+n-r)+(4+r)+(8+n-r)+7-1-1 \\
& =6 n+25-4 r .
\end{aligned}
$$

Apart from the -1 corresponding to the overall rescaling, one more -1 is performed, because we can choose the leading coefficient of $t_{r}$ as 1 when factorizing $h_{n}=h_{n-r} t_{r}$ and $h_{n}^{\prime}=h_{n-r}^{\prime} t_{r}$. If we counted the degrees of freedom of $h_{n-r}$ as $n-r+1$ and those of $t_{r}$ as $r+1$, it would be an overcounting.

The charged matter spectrum is derived in appendix C.1 by using the series expansions of $f_{\text {res }}, g_{\text {res }}$ and $\Delta_{\text {res }}$ near each of the three lines $z=0, \tilde{z}=0$ and $\hat{z}=0$. The resulting charged matter multiplets (C.5) together with the singlets (5.48) give the total spectrum as

$$
\begin{aligned}
6 n+25-4 r & :(\mathbf{1}, \mathbf{1}, \mathbf{1}) \\
2 n+16+2 r & :(\mathbf{2}, \mathbf{1}, \mathbf{1}),(\mathbf{1}, \mathbf{2}, \mathbf{1}),(\mathbf{1}, \mathbf{1}, \mathbf{2}) \\
n-r & :(\mathbf{2}, \mathbf{2}, \mathbf{1}),(\mathbf{2}, \mathbf{1}, \mathbf{2}),(\mathbf{2}, \mathbf{2}, \mathbf{1}) \\
r & : \frac{1}{2}(\mathbf{2}, \mathbf{2}, \mathbf{2})
\end{aligned}
$$

which perfectly reproduces the heterotic result (table 9$).{ }^{15}$

A brief sketch for the structure of the symmetry enhancement is depicted in figure 1. The curves express the discriminant locus $\Delta_{\text {res }}=0$ (the shapes are not accurate). Each matter is localized at each intersection point.

\footnotetext{
${ }^{15}$ The procedure of constructing geometry for general instanton distribution described in this subsection is applicable to other cases where the gauge bundle is divided into components $H=\otimes_{i} H_{i}$. We have studied models whose $H_{i}$ 's are all Cartan types. There are 7 such cases out of 74 Oguiso-Shioda classification: Nos.7,10,11,14,15,18 and 26. Among them, $\mathrm{CY}_{3}$ of No.15 and No.26 have been constructed in [60]. We have examined the other cases and have constructed the $\mathrm{CY}_{3}$ of Nos.7,10,11 and 18 with general instanton distributions. For each case, the F-theoretic spectrum precisely coincides with the heterotic spectrum. However, we have not succeeded to construct $\mathrm{CY}_{3}$ for No.14 yet.
} 

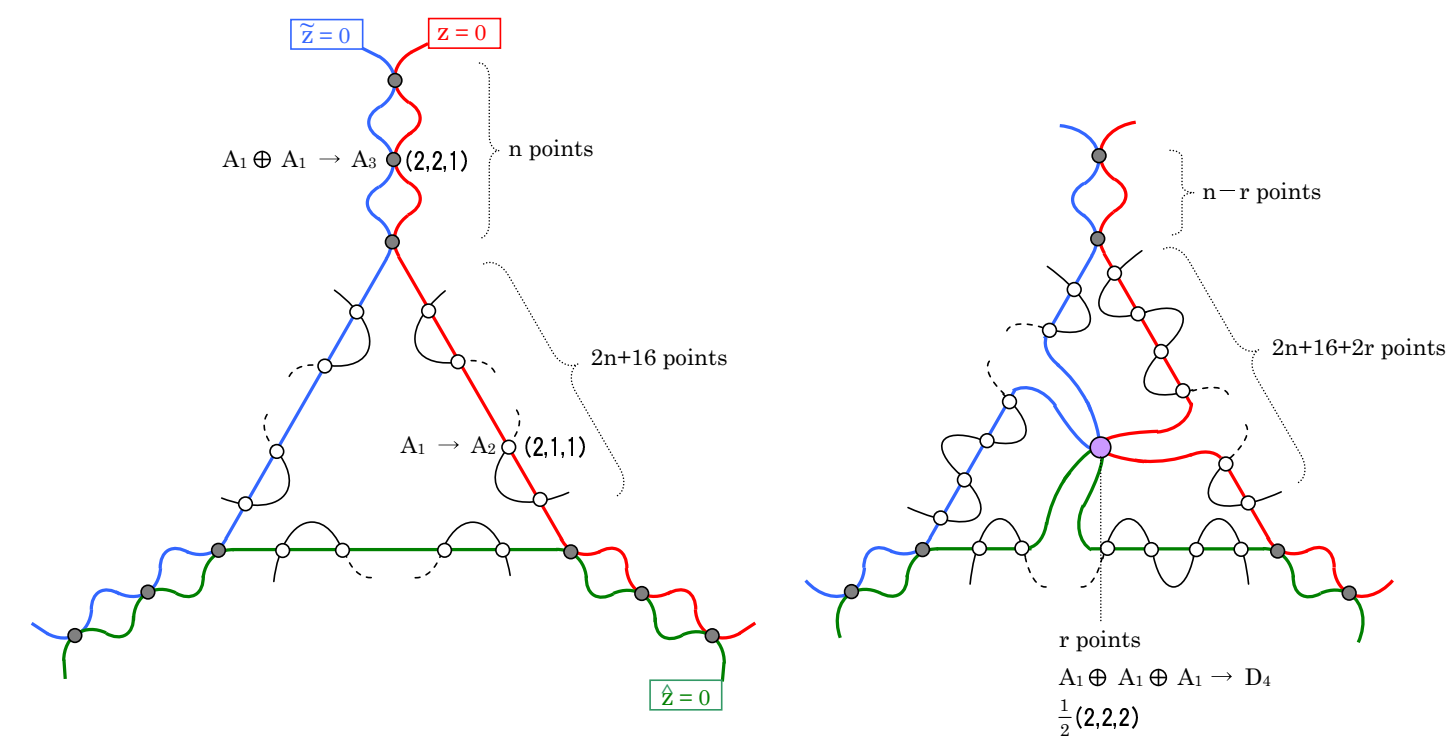

Figure 1. Discriminant locus for $G=\mathrm{SU}(2) \times \mathrm{SU}(2) \times \mathrm{SU}(2)$ (No.7): $r=0$ (left) and $r \neq 0$ (right).

\subsection{Looijenga's theorem}

Our geometry not only reproduces the heterotic spectrum but also encodes the structure of the moduli space $\mathcal{M}(H)$ of the gauge bundle $H$. On the heterotic side, Looijenga's theorem claims that the moduli space $\mathcal{M}(H)$ is parameterized by the sections (we consider the six-dimensional case)

$$
\omega_{k} \in \Gamma\left(\mathcal{L}^{-d_{k}} \times \mathcal{N}^{s_{k}}\right) \quad(k=0, \ldots, \operatorname{rank} G) .
$$

Here $d_{k}$ is the degree of the independent Casimir of $H\left(d_{0} \equiv 0\right.$ for $\left.k=0\right)$, and $s_{k}$ is the coefficient of the $k$-th coroot when the lowest root $-\theta$ is expanded $\left(s_{0} \equiv 1\right.$ for $\left.k=0\right)$. $\mathcal{L}$ is the anti-canonical line bundle of the base $\mathbb{P}^{1}$ of the heterotic K3 and $\mathcal{N}$ is the "twisting" line bundle over $\mathbb{P}^{1}$. Explicitly,

$$
\begin{aligned}
\mathcal{L} & =\mathcal{O}(2), \\
\mathcal{N} & =\mathcal{O}(p),
\end{aligned}
$$

where $p$ corresponds to the instanton number. Therefore, degrees of $\omega_{k}$ with respect to the coordinate $z^{\prime}$ of $\mathbb{P}^{1}$ is given by

$$
\operatorname{deg}\left(\omega_{k}\right)=p s_{k}-2 d_{k} .
$$

$z^{\prime}$ is identified with the coordinate of the base $\mathbb{P}^{1}$ of $F_{n}$, i.e., the variable of polynomials $a_{i j}$.

In the present case, $H=\mathrm{SO}(8) \times \mathrm{SU}(2)$ with instanton numbers $(8+n-r, 4+r)$. Then we find that the sections $\omega_{k}$ in Looijenga's theorem exactly match the 7 independent polynomials (5.47) describing the geometry on the F-theory side (see table 10).

This correspondence is also valid for $r=0$. When $r=0$, the order of the polynomial $t_{r}$ becomes 0 and the independent polynomials are reduced to those of the $r=0$ case (5.20). In other words, the geometry for the $r=0$ case already captures the structure of the moduli space of the bundle $H$. In the following discussion, we concentrate on the $r=0$ case. 


\begin{tabular}{|c|c|cll|l|}
\hline$H$ & $p$ & $s_{k}$ & $d_{k}$ & $\operatorname{deg}\left(\omega_{k}\right)$ & Polynomial \\
\hline $\mathrm{SO}(8)$ & $8+n-r$ & 1 & 0 & $8+n-r$ & $j_{8+n-r}$ \\
& & 1 & 2 & $4+n-r$ & $k_{4+n-r}$ \\
& & 1 & 4 & $n-r$ & $h_{n-r}$ \\
& & 1 & 4 & $n-r$ & $h_{n-r}^{\prime}$ \\
& & 2 & 6 & $4+2 n-2 r$ & $s_{4+2 n-2 r}$ \\
\hline $\mathrm{SU}(2)$ & $4+r$ & 1 & 0 & $4+r$ & $q_{4+r}$ \\
& & 1 & 2 & $r$ & $t_{r}$ \\
\hline
\end{tabular}

Table 10. Looijenga's theorem.

\section{Geometry for non-Cartan $\mathrm{SU}(7)$ series}

We have constructed the $\mathrm{CY}_{3}$ for $G=\mathrm{SU}(2) \times \mathrm{SU}(2) \times \mathrm{SU}(2)$ in the previous section. This is No.7 and is not contained in table 1, but by unHiggsing the first $\mathrm{SU}(2)$ successively, we obtain the $\mathrm{CY}_{3}$ for No.12 in the $\mathrm{SU}(7)$ series and the $\mathrm{CY}_{3}$ for No.22 in the $\mathrm{SU}(8)$ series as

$$
\mathrm{SU}(2) \times \mathrm{SU}(2) \times \mathrm{SU}(2)_{(7)} \rightarrow \mathrm{SU}(3) \times \mathrm{SU}(2) \times \mathrm{SU}(2)_{(12)} \rightarrow \mathrm{SU}(4) \times \mathrm{SU}(2) \times \mathrm{SU}(2)_{(22)} .
$$

$\mathrm{CY}_{3}$ 's for other cases can be obtained by further unHiggsing them. Once we obtain a $\mathrm{CY}_{3}$ for each case, we then map it to a RES-fibered geometry, extract the matter spectrum and compare it with the heterotic result given in section 3, Appendix A and appendix B.

To start the construction from No.7, we focus on the $r=0$ case. It turns out that starting from the geometry with $r \neq 0$ causes technical difficulties; in this paper, we do not consider such cases.

\section{1 $G=\mathrm{SU}(3) \times \mathrm{SU}(2) \times \mathrm{SU}(2)(\mathrm{No.12})$}

Let us enhance the first $\mathrm{SU}(2)$ of $G=\mathrm{SU}(2) \times \mathrm{SU}(2) \times \mathrm{SU}(2)$ to $\mathrm{SU}(3)$. The discriminant for $G=\mathrm{SU}(2) \times \mathrm{SU}(2) \times \mathrm{SU}(2)$ with $r=0$ is given by $\Delta=z^{2}\left(z+h_{n}\right)^{2}\left(z+h_{n}^{\prime}\right)^{2} D_{6 n+24}$ (see (5.17)). To enhance the first factor, we impose that $D_{6 n+24}$ is factorized as

$$
D_{6 n+24}=z \cdot E_{5 n+24},
$$

where $E_{5 n+24}$ should be an irreducible polynomial. For this, we require that the constant term of $D_{6 n+24}$ vanishes. From (5.18), the condition is

$$
A_{20}^{2}\left(4 A_{20} A_{22}^{3}-27 A_{43}^{2}+36 A_{20} A_{22} \tilde{A}_{44}+108 A_{20} \tilde{A}_{66}\right)=0 .
$$

A solution is $A_{20}=0$, but it is not suitable. As seen from the explicit form of $f$ and $g$ (5.15), $A_{20}=0$ enhances the orders not only of $\Delta$ but also of $f$ and $g$ near $z=0$ to be $(\operatorname{ord}(f), \operatorname{ord}(g), \operatorname{ord}(\Delta))=(1,2,3)$. The fiber degeneracy enhances as $I_{2} \rightarrow I I I$, but the singularity is kept fixed as $A_{1} \rightarrow A_{1}$, not enhance to $A_{2}$. Thus we have to require the other factor vanishes. Namely,

$$
-27 A_{43}^{2}+4 A_{20}\left(A_{22}^{3}+9 A_{22} \tilde{A}_{44}+27 \tilde{A}_{66}\right)=0 .
$$


Since it contains the squire $A_{43}^{2}$, the remaining part $4 A_{20}\left(A_{22}^{3}+9 A_{22} \tilde{A}_{44}+27 \tilde{A}_{66}\right)$ should be a perfect square. There are two solutions. First one is $\tilde{A}_{66}=-\frac{1}{27}\left(A_{22}^{3}+9 A_{22} \tilde{A}_{44}\right)+A_{20} p_{4-n}^{2}$, and then $A_{43}$ is solved as $A_{43}=2 A_{20} p_{4-n}$. However, one can check that the split condition is not satisfied by this solution. The split condition for $\mathrm{SU}(3)$ says that the coefficient of $z^{3}$ in $\Delta$ should be proportional to $a_{10}^{3}$ (see table 8 ), but in this solution it is proportional to $A_{20}^{3}$, as one can see by calculating the next to leading order term of $D_{6 n+24}$ in (5.18). The other solution is

$$
\begin{aligned}
& A_{20}=p_{2+n}^{2}, \\
& \tilde{A}_{66}=-\frac{1}{27}\left(A_{22}^{3}+9 A_{22} \tilde{A}_{44}\right)+q_{6}^{2},
\end{aligned}
$$

and then

$$
A_{43}=2 p_{2+n} q_{6}
$$

In this case, the coefficient of $z^{3}$ in the expansion of $\Delta$ is proportional to $p_{2+n}^{3}$, and hence the split condition is fulfilled. Substituting (6.5) and (6.6) into (5.18), we obtain the explicit form of $E_{5 n+24}(6.2)$ as

$$
\begin{aligned}
E_{5 n+24}= & \frac{4}{3} p_{2+n}^{3}\left(-A_{22}^{2} p_{2+n}^{2} q_{6}+3 A_{21} p_{2+n} q_{6}^{2}-3 \tilde{A}_{44} p_{2+n}^{2} q_{6}+A_{22} A_{45} p_{2+n}^{3}+3 \tilde{A}_{67} p_{2+n}^{3}-3 q_{6}^{3} \sigma_{2}\right) \\
& +o(z) .
\end{aligned}
$$

One can check that $E_{5 n+24}$ is irreducible and the gauge symmetry is in fact $\mathrm{SU}(3) \times \mathrm{SU}(2) \times$ $\mathrm{SU}(2)$.

Mapping $f$ and $g$ to $f^{\prime}$ and $g^{\prime}$, and setting the polynomials (5.19) to zero in $f^{\prime}$ and $g^{\prime}$, one obtains the RES-fibered geometry with $G=\mathrm{SU}(3) \times \mathrm{SU}(2) \times \mathrm{SU}(2)$. The explicit forms of $f_{\text {res }}, g_{\text {res }}$ and $\Delta_{\text {res }}$ are given in appendix C.2. In this way, we obtain the geometry for the non-Cartan Mordell-Weil lattice $E(K)=\Lambda_{12}$ in table 1 .

Let us extract the matter spectrum from the resulting geometry. We first notice that $f_{\text {res }}$ and $g_{\text {res }}$ contain the following six independent polynomials:

\begin{tabular}{|c|c|}
\hline Polynomial & Degree \\
\hline$h_{n}$ & $n$ \\
$h_{n}^{\prime}$ & $n$ \\
$p_{2+n}$ & $2+n$ \\
$A_{21}$ & $4+n$ \\
$A_{22}$ & 4 \\
$q_{6}$ & 6 \\
\hline
\end{tabular}

For counting the number of singlets, it should be noted that the middle polynomial $\tilde{A}_{66}$ is written in terms of these polynomials as in (6.5). In other words, these six polynomials include the degrees of freedom of $\tilde{A}_{66}$, which are the geometric moduli of K3 on the heterotic side. In order to focus on the gauge bundle moduli only, we subtract 13 degrees of freedom corresponding to $\tilde{A}_{66}$ and return it to the geometric moduli of K3. As a result, we obtain

$$
n\left(H_{0}\right)=n+n+(2+n)+(4+n)+(4)+(6)+6-1-13=4 n+8 .
$$


The derivation of the charged matter spectrum is given in appendix C.2 and the result is summarized in (C.10). Together with the singlets (6.9), the full spectrum is given by

$$
\begin{aligned}
4 n+8 & :(\mathbf{1}, \mathbf{1}, \mathbf{1}) \\
2 n+18 & :(\mathbf{3}, \mathbf{1}, \mathbf{1}) \\
n+16 & :(\mathbf{1}, \mathbf{2}, \mathbf{1}) \\
n+16 & :(\mathbf{1}, \mathbf{1}, \mathbf{2}) \\
n & :(\mathbf{3}, \mathbf{2}, \mathbf{1}) \\
n & :(\mathbf{3}, \mathbf{1}, \mathbf{2}) \\
n & :(\mathbf{1}, \mathbf{2}, \mathbf{2})
\end{aligned}
$$

This F-theoretic spectrum is equivalent to the heterotic spectrum (see appendix A.2) given in table 15 with $r_{\mathrm{U}(1)}^{\prime}=2$, or the one given in table 16 with $\left(r, \tilde{r}, r_{(17)}\right)$ for $k=2$ in (A.19).

\section{$6.2 \quad G=\mathrm{SU}(5) \times \mathrm{SU}(2)(\mathrm{No.17})$}

The discriminant of $\mathrm{CY}_{3}$ for $G=\mathrm{SU}(3) \times \mathrm{SU}(2) \times \mathrm{SU}(2)$ is $\Delta=z^{3}\left(z+h_{n}\right)^{2}\left(z+h_{n}^{\prime}\right)^{2} E_{5 n+24}$ (see (6.2)). One expect that tuning the parameters $h_{n}, h_{n}^{\prime}$ causes the unHigssing process as follows:

$$
\begin{array}{ll}
h_{n}^{\prime}=0 & \rightarrow G=\mathrm{SU}(5) \times \mathrm{SU}(2), \\
h_{n}^{\prime}=h_{n} & \rightarrow G=\mathrm{SU}(3) \times \mathrm{SU}(4), \\
h_{n}^{\prime}=h_{n}=0 & \rightarrow G=\mathrm{SU}(7) .
\end{array}
$$

In the first case, the tuning gives the expected enhancement. However, in the second and third cases, naïve tunings give a non-split fiber and unresolvable singularities, respectively, and hence we need some modifications of the geometry.

In this subsection, we consider the $G=\mathrm{SU}(5) \times \mathrm{SU}(2)$ case. The $\mathrm{CY}_{3}$ is obtained by merely setting

$$
h_{n}^{\prime}=0
$$

in the formulae obtained in the previous section.

We then map $f$ and $g$ to $f^{\prime}$ and $g^{\prime}$. The resulting $f^{\prime}$ and $g^{\prime}$ contain polynomials

$$
A_{45}, A_{46}, A_{47}, \tilde{A}_{67}, \tilde{A}_{68}
$$

which constitute the coefficients of the terms higher than $o\left(z^{4}\right)$ in $f$ and $o\left(z^{6}\right)$ in $g$. After setting them to zero, one obtains the RES-fibered geometry with $G=\mathrm{SU}(5) \times \mathrm{SU}(2)$. The explicit forms of $f_{\text {res }}, g_{\text {res }}$ and $\Delta_{\text {res }}$ are given in appendix C.3. In this way, we obtain the geometry for the non-Cartan Mordell-Weil lattice $E(K)=\Lambda_{17}$ in table 1.

The number of singlets is reduced by $n+1$ ( of $h_{n}^{\prime}$ ) from (6.9):

$$
n\left(H_{0}\right)=4 n+8-(n+1)=3 n+7 .
$$


The charged matter spectrum is derived in appendix C.3 and given in (C.14). Together with the singlets (6.14), the full spectrum is given by

$$
\begin{aligned}
3 n+7 & :(\mathbf{1}, \mathbf{1}) \\
n+16 & :(\mathbf{5}, \mathbf{1}) \\
n+16 & :(\mathbf{1}, \mathbf{2}) \\
n+2 & :(\mathbf{1 0}, \mathbf{1}) \\
n & :(\mathbf{5}, \mathbf{2})
\end{aligned}
$$

This F-theoretic spectrum is equivalent to the heterotic spectrum (see section 3.4) given in table 4 with $(r, \tilde{r})$ for $k=2$ in $(3.24){ }^{16}$

\section{$6.3 \quad G=\mathrm{SU}(3) \times \mathrm{SU}(4)(\mathrm{No} .19)$}

Let us impose on the $\mathrm{CY}_{3}$ with $G=\mathrm{SU}(3) \times \mathrm{SU}(2) \times \mathrm{SU}(2)$ the condition

$$
h_{n}^{\prime}=h_{n}
$$

to obtain a $\mathrm{CY}_{3}$ with $G=\mathrm{SU}(3) \times \mathrm{SU}(4)$. This tuning, however, breaks the split condition. By the replacement $h_{n}^{\prime}=h_{n}$, one expects the $\mathrm{SU}(4)$ singularity appears at $\tilde{z}=z+h_{n}=0$. One can show that the leading expansion of $\Delta$ near $\tilde{z}=0$ takes the form

$$
\Delta=\left(p_{2+n}^{2}-A_{21} h_{n}+A_{22} h_{n}^{2}\right)^{2} h_{n}^{3} \tilde{T}_{16+n} \tilde{z}^{4}+\cdots,
$$

which is different from the split condition for $\mathrm{SU}(4)$ (see table 8)

$$
\Delta=-\frac{1}{16} a_{10}^{4} b_{84} \tilde{z}^{4}+\cdots
$$

To fulfill this condition, we have to require the perfect square form for the factor in (6.17) as

$$
p_{2+n}^{2}-A_{21} h_{n}+A_{22} h_{n}^{2}=\tilde{p}_{2+n}^{2}
$$

with some order $2+n$ polynomial $\tilde{p}_{2+n}$. For this, we need a term linear of $p_{2+n}$ in the l.h.s. Let us write

$$
A_{21}=p_{n+2} s_{2}+h_{n} r_{4} .
$$

Then the 1.h.s. of (6.19) is perfect squire if and only if

$$
r_{4}=A_{22}-\frac{1}{4} s_{2}^{2}
$$

and $\tilde{p}_{2+n}$ is solved as

$$
\tilde{p}_{2+n}=p_{2+n}-\frac{1}{2} s_{2} h_{n}
$$

\footnotetext{
${ }^{16}$ This F-theoretic spectrum does not coincide with the heterotic spectrum given in table 6 , which is coarser than the one in table 4. In order to obtain the corresponding geometry, we have to deform the one we found here in response to the change of the distribution of instantons, as we did for $G=\mathrm{SU}(2) \times$ $\mathrm{SU}(2) \times \mathrm{SU}(2)$, but so far, such a geometry has not been found.
} 
Therefore, unHiggsing to $\mathrm{SU}(3) \times \mathrm{SU}(4)$ is given by imposing on the $\mathrm{CY}_{3}$ of $\mathrm{SU}(3) \times$ $\mathrm{SU}(2) \times \mathrm{SU}(2)$ not only (6.16) but also (6.20) with (6.21).

Mapping $f$ and $g$ to $f^{\prime}$ and $g^{\prime}$, and setting the polynomials (5.19) to zero in $f^{\prime}$ and $g^{\prime}$, one obtains the RES-fibered geometry with $G=\mathrm{SU}(3) \times \mathrm{SU}(4)$. The explicit forms of $f_{\text {res }}, g_{\text {res }}$ and $\Delta_{\text {res }}$ are given in appendix C.4. In this way, we obtain the geometry for the non-Cartan Mordell-Weil lattice $E(K)=\Lambda_{19}$ in table 1 .

In the table (6.8) of the independent polynomials, $h_{n}^{\prime}$ is eliminated and $A_{21}$ is replaced by $s_{2}$, which reduce the degrees of freedom by $(n+1)+(5+n-3)=2 n+3$ from (6.9). The number of singlets is hence given by

$$
n\left(H_{0}\right)=4 n+8-(2 n+3)=2 n+5 .
$$

The charged matter spectrum is derived in appendix C.4 and given in (C.18). Together with the singlets (6.23), the full spectrum is given by

$$
\begin{aligned}
2 n+5 & :(\mathbf{1}, \mathbf{1}) \\
2 n+18 & :(\mathbf{3}, \mathbf{1}) \\
n+16 & :(\mathbf{1}, \mathbf{4}) \\
n+2 & :(\mathbf{1}, \mathbf{6}) \\
n & :(\mathbf{3}, \mathbf{4})
\end{aligned}
$$

This F-theoretic spectrum is equivalent to the heterotic spectrum (see appendix A.1) given in table 12 with $(r, \tilde{\tilde{r}})$ for $k=2$ in (A.6), or the one given in table 14 with $r_{\mathrm{U}(1)}^{\prime}=1$.

\section{$6.4 \quad G=\mathrm{SU}(7)(\mathrm{No} .25)$}

To obtain a $\mathrm{CY}_{3}$ for $G=\mathrm{SU}(7)$, we impose on the $\mathrm{CY}_{3}$ for $G=\mathrm{SU}(3) \times \mathrm{SU}(4)$ derived above a further condition

$$
h_{n}=0 .
$$

However, an explicit calculation tells us that it contains unresolvable singularities. Namely, $(\operatorname{ord}(f), \operatorname{ord}(g), \operatorname{ord}(\Delta))=(4,6,12)$ at $p_{2+n}=0$.

To make the singularity milder, we have to do some redefinition of polynomials and "absorb" the factors of $p_{2+n}$. For this, look at the explicit form of $f$ :

$$
\begin{aligned}
f= & -\frac{1}{3} p_{2+n}^{4}-\frac{2}{3} p_{2+n}^{3} s_{2} z-\frac{1}{3}\left(2 A_{22} p_{2+n}^{2}+p_{2+n}^{2} s_{2}^{2}\right) z^{2}+\frac{2}{3}\left(3 p_{2+n} q_{6}-A_{22} p_{2+n} s_{2}\right) z^{3} \\
& +\tilde{A}_{44} z^{4}+A_{45} z^{5}+A_{46} z^{6}+A_{47} z^{7}+A_{48} z^{8}
\end{aligned}
$$

where the order is enhanced to $\operatorname{ord}(f)=4$ at $p_{2+n}=0$. Here, one can notice that $s_{2}$ always appears as a product form $p_{2+n} s_{2}$. We can hence introduce a new polynomial $r_{4+n}$ and replace $s_{2}$ as

$$
s_{2}=\frac{r_{4+n}}{p_{2+n}}
$$

The expansion of $f$ is rewritten as

$$
f=-\frac{1}{3} p_{2+n}^{4}-\frac{2}{3} p_{2+n}^{2} r_{4+n} z-\frac{1}{3}\left(2 A_{22} p_{2+n}^{2}+r_{4+n}^{2}\right) z^{2}+\cdots,
$$


whose order at $p_{2+n}=0$ is suppressed to ord $(f)=2$. Similar calculation for $g$ and $\Delta$ shows that the singularity at $p_{2+n}=0$ gets milder as $(\operatorname{ord}(f), \operatorname{ord}(g), \operatorname{ord}(\Delta))=(2,3,9)$, which is $D_{5}$ singularity and can be resolved without any problem.

Therefore, unHiggsing to $G=\mathrm{SU}(7)$ is obtained by imposing (6.25) and (6.27) on the $\mathrm{CY}_{3}$ for $G=\mathrm{SU}(3) \times \mathrm{SU}(4) .{ }^{17}$

We then map $f$ and $g$ to $f^{\prime}$ and $g^{\prime}$. The resulting $f^{\prime}$ and $g^{\prime}$ contain polynomials $A_{45}$ and $A_{46}$, which constitute the coefficients of the terms higher than $o\left(z^{4}\right)$ in $f$ and $o\left(z^{6}\right)$ in $g$. After setting them to zero, one obtains a RES-fibered geometry with $G=\mathrm{SU}(7)$. The explicit forms of $f_{\text {res }}, g_{\text {res }}$ and $\Delta_{\text {res }}$ are given in appendix C.5. In this way, we obtain the geometry for the non-Cartan Mordell-Weil lattice $E(K)=\Lambda_{25}$ in table 1 .

Among the independent polynomials for $G=\mathrm{SU}(3) \times \mathrm{SU}(4), h_{n}$ is eliminated and $s_{2}$ is replaced by $r_{4+n}$. The degrees of freedom (6.23) is reduced by $(n+1)+\{3-(5+n)\}=-1$, that is, increases by 1 :

$$
n\left(H_{0}\right)=2 n+5+1=2 n+6 .
$$

The charged matter spectrum is derived in appendix C.5 and given in (C.21). Together with the singlets (6.29), the full spectrum is given by

$$
\begin{aligned}
2 n+6 & : \mathbf{1} \\
n+16 & : \mathbf{7} \\
n+2 & : \mathbf{2 1}
\end{aligned}
$$

This F-theoretic spectrum is equivalent to the heterotic spectrum (see section 3.2) given in table 2 with $r^{\prime}=1$.

\section{Geometry for non-Cartan SU(8) series}

\section{$7.1 G=\mathrm{SU}(4) \times \mathrm{SU}(2) \times \mathrm{SU}(2)(\mathrm{No.22})$}

In section 6.1, we constructed a $\mathrm{CY}_{3}$ for $G=\mathrm{SU}(3) \times \mathrm{SU}(2) \times \mathrm{SU}(2)$. The discriminant has the form $\Delta=z^{3}\left(z+h_{n}\right)^{2}\left(z+h_{n}^{\prime}\right)^{2} E_{5 n+24}$. The enhancement $\mathrm{SU}(3) \rightarrow \mathrm{SU}(4)$ occurs if it factorizes as

$$
E_{5 n+24}=z \cdot F_{4 n+24}
$$

where $F_{4 n+24}$ should be an irreducible polynomial. The explicit form of $E_{5 n+24}$ is given in (6.7). One way to achieve (7.1) is to set $p_{2+n}=0$ so that the $o(1)$ term of (6.7) vanishes. This does not, however, lead to the enhancement to $\mathrm{SU}(4)$ since in this case we have $(\operatorname{ord}(f), \operatorname{ord}(g), \operatorname{ord}(\Delta))=(2,2,4)$, which means that the fiber type is $I V$, and hence the singularity does not change $A_{2} \rightarrow A_{2}$. Thus we must require the other factor of the $o(1)$ term to vanish:

$$
-A_{22}^{2} p_{2+n}^{2} q_{6}+3 A_{21} p_{2+n} q_{6}^{2}-3 \tilde{A}_{44} p_{2+n}^{2} q_{6}+A_{22} A_{45} p_{2+n}^{3}+3 \tilde{A}_{67} p_{2+n}^{3}-3 q_{6}^{3} h_{n} h_{n}^{\prime}=0 .
$$

Here we used $\sigma_{2}=h_{n} h_{n}^{\prime}$.

\footnotetext{
${ }^{17}$ This $\mathrm{CY}_{3}$ is equivalent to the one constructed in [66] with $r=0\left(\delta=1\right.$ and $\left.\zeta_{1}=0\right)$. The dictionary is as follows: $p_{2+n}=\frac{1}{2} \xi, r_{4+n}=\zeta_{2}, A_{22}=\omega, q_{6}=-3 \lambda_{1}, \tilde{A}_{44}=\psi_{4}-\frac{1}{12} \omega^{2}, A_{4 j}=f_{j}(5 \leq j \leq 8)$, $\tilde{A}_{67}=g_{7}+\frac{1}{3} \zeta_{2} f_{6}+\frac{1}{12} \xi^{2} f_{7}, \tilde{A}_{68}=g_{8}+\frac{1}{3} \zeta_{2} f_{7}+\frac{1}{12} \xi^{2} f_{8}, \tilde{A}_{69}=g_{9}+\frac{1}{3} \zeta_{2} f_{8}, \tilde{A}_{6,10}=g_{10}$ and $A_{6 j}=g_{j}$ $(j=10,11)$.
} 
There are two solutions to (7.2). One solution is given by

$$
\begin{aligned}
q_{6} & =t_{4-n} p_{2+n}, \\
\tilde{A}_{67} & =-\frac{1}{3}\left(A_{22} A_{45}-A_{22}^{2} t_{4-n}-3 \tilde{A}_{44} t_{4-n}+3 A_{21} t_{4-n}^{2}-3 h_{n} h_{n}^{\prime} t_{4-n}^{3}\right) .
\end{aligned}
$$

Let us call this $\mathrm{CY}_{3}$, "CY$(1)$ ". In this solution, however, $\tilde{A}_{67}$, which is a coefficient of a term higher than $o\left(z^{6}\right)$ in $g$, contains terms written by only the polynomials which are needed to express $f_{\text {res }}$ and $g_{\text {res }}$. This means that, to achieve the singularities, it is not sufficient to constrain the terms of $o\left(z^{4}\right)$ or lower in $f$ and of $o\left(z^{6}\right)$ or lower in $g$, but we also need to impose conditions on the higher order terms, which are supposed to be independently tuned to describe the instanton bundle of the "other" $E_{8}$ gauge group of the dual heterotic theory. Thus, in the same way as in the $G=\mathrm{SU}(8)$ case (4.23) discussed in section 4.2, the map from $\mathrm{CY}_{3}^{(1)}$ to a RES-fibered geometry changes the singularity.

The other solution can be found as follows. From the form of (7.2), we can see that it is solved for $A_{21}$ by requiring that all the terms have a common factor of $p_{2+n} q_{6}^{2}$. This is possible if ${ }^{18}$

$$
\begin{aligned}
p_{2+n} & =r_{n-4} q_{6}, \\
h_{n} & =r_{n-4} s_{4} .
\end{aligned}
$$

The solution is given by

$$
A_{21}=\frac{1}{3}\left(A_{22}^{2}+3 \tilde{A}_{44}-A_{22} A_{45} r_{n-4}-3 \tilde{A}_{67} r_{n-4}\right) r_{n-4}+h_{n}^{\prime} s_{4}
$$

This $\mathrm{CY}_{3}$, which we call $\mathrm{CY}_{3}^{(2)}$, can be mapped to a RES-fibered geometry, where the singularity is unchanged. Mapping $f$ and $g$ to $f^{\prime}$ and $g^{\prime}$ and setting the polynomials (5.19) contained in $f^{\prime}$ and $g^{\prime}$, one obtains the RES-fibered geometry with $G=\mathrm{SU}(4) \times \mathrm{SU}(2) \times$ $\mathrm{SU}(2)$. The explicit form of $f_{\text {res }}, g_{\text {res }}$ and $\Delta_{\text {res }}$ are given in appendix C.6. In this way, we obtain the geometry for the non-Cartan Mordell-Weil lattice $E(K)=A_{1}^{*} \oplus A_{1}^{*} \oplus\langle 1 / 4\rangle$ in table 1.

The independent polynomials for $G=\mathrm{SU}(3) \times \mathrm{SU}(2) \times \mathrm{SU}(2)(6.8)$ are reduced by (7.4) and (7.5) to the following 5 polynomials:

\begin{tabular}{|c|c|}
\hline Polynomial & Degree \\
\hline$r_{n-4}$ & $n-4$ \\
$h_{n}^{\prime}$ & $n$ \\
$s_{4}$ & 4 \\
$A_{22}$ & 4 \\
$q_{6}$ & 6 \\
\hline
\end{tabular}

The number of singlets is given by

$$
n\left(H_{0}\right)=(n-4)+n+(4)+(4)+(6)+5-1-13=2 n+1 .
$$

Here we subtracted the degree 13 of $\tilde{A}_{66}$ for the same reason as in (6.9).

\footnotetext{
${ }^{18}$ We can use $h_{n}^{\prime}=r_{n-4} s_{4}^{\prime}$ instead of the second equation. The resulting spectrum is equivalent.
} 
The charged matter spectrum is derived in appendix C.6 and given in (C.26). Together with the singlets (7.7), the full spectrum is given by

$$
\begin{aligned}
2 n+1 & :(\mathbf{1}, \mathbf{1}, \mathbf{1}) \\
2 n+8 & :(\mathbf{4}, \mathbf{1}, \mathbf{1}) \\
6 & :(\mathbf{6}, \mathbf{1}, \mathbf{1}) \\
n+12 & :(\mathbf{1}, \mathbf{2}, \mathbf{1}) \\
16 & :(\mathbf{1}, \mathbf{1}, \mathbf{2}) \\
4 & :(\mathbf{4}, \mathbf{2}, \mathbf{1}) \\
n & :(\mathbf{4}, \mathbf{1}, \mathbf{2}) \\
n & :(\mathbf{1}, \mathbf{2}, \mathbf{2}) \\
n-4 & : \frac{1}{2}(\mathbf{6}, \mathbf{2}, \mathbf{1})
\end{aligned}
$$

This F-theoretic spectrum is equivalent to the heterotic spectrum (see appendix B.2) given in table 20 with $(r, \tilde{r})=(4, n)$.

Two comments are in order. First, it is difficult to construct this geometry in Tate's form. Recall the construction for $G=\mathrm{SU}(2) \times \mathrm{SU}(2) \times \mathrm{SU}(2)$ given in section 5 . As seen in (5.11), $a_{i}(z)$ contains $a_{i j}$ with orders greater than or equal to ord $\left(a_{i}\right)=(0,0,3,3,6)$. For example, $a_{4}(z)$ contains $a_{43}, a_{44}, \ldots, a_{48}$. These orders are determined by the sum of the orders of each SU(2) factor. Since an $\mathrm{SU}(2)$ singularity has $\operatorname{ord}\left(a_{i}\right)=(0,0,1,1,2), \mathrm{SU}(2) \times$ $\mathrm{SU}(2) \times \mathrm{SU}(2)$ gives ord $\left(a_{i}\right)=(0,0,3,3,6)$. Similar counting for $\mathrm{SU}(4) \times \mathrm{SU}(2) \times \mathrm{SU}(2)$ gives $\operatorname{ord}\left(a_{i}\right)=(0,1,4,4,8)$. These orders are equal to those for $\mathrm{SU}(8)$. In particular, $\operatorname{ord}\left(a_{3}\right)$ and $\operatorname{ord}\left(a_{6}\right)$ exceed 3 and 6 . As we have explained in section 4.2 , it leads to a factorized form of the discriminant $\Delta_{\text {res }}=a_{4}^{2}\left(4 a_{4}-b_{2}^{2}\right)$, yielding unwanted additional singularities. ${ }^{19}$

Second comment is: $\mathrm{CY}_{3}^{(1)}$ of the first solution (7.3), which cannot be mapped to a RES-fibered geometry, is related to the known $\mathrm{CY}_{3}$ (4.23) for $G=\mathrm{SU}(8)$ [66]. In fact, setting $h_{n}=h_{n}^{\prime}=0$ in $\mathrm{CY}_{3}^{(1)}$ causes the unHiggsing process $\mathrm{SU}(4) \times \mathrm{SU}(2) \times \mathrm{SU}(2) \rightarrow \mathrm{SU}(8)$, and the resulting $\mathrm{CY}_{3}$ precisely coincides with (4.23). ${ }^{20}$ In other words, the $\mathrm{CY}_{3}^{(2)}$ we have constructed in this paper, without which one can never make a connection with the theory of the Mordell-Weil lattice, belongs to a new branch different from the one connected to the known $\mathrm{CY}_{3}$ with $G=\mathrm{SU}(8)$ of $[66] .^{21}$

\section{$7.2 \quad G=\mathrm{SU}(6) \times \mathrm{SU}(2)(\mathrm{No} .29)$}

As seen from (7.1) and (7.4), the discriminant of $\mathrm{CY}_{3}$ for $G=\mathrm{SU}(4) \times \mathrm{SU}(2) \times \mathrm{SU}(2)$ is $\Delta=z^{4}\left(z+r_{n-4} s_{4}\right)^{2}\left(z+h_{n}^{\prime}\right)^{2} F_{2 n+24}$. By tuning the polynomials $r_{n-4}, s_{4}$ and $h_{n}^{\prime}$ suitably, one may expect to continue similar unHiggsing processes.

\footnotetext{
${ }^{19}$ As for the $\mathrm{SU}(7)$ series, they can be constructed by Tate's form. For example, the counting of the orders for $\mathrm{SU}(3) \times \mathrm{SU}(2) \times \mathrm{SU}(2)$ gives ord $\left(a_{i}\right)=(0,1,3,4,7)$, and hence $a_{33}$ remains non-zero in $f_{\text {res }}$ and

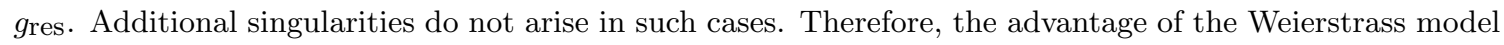
starts from $\mathrm{SU}(8)$ series.

${ }^{20}$ The dictionary is as follows: $p_{2+n}=\frac{1}{2} \tau, A_{21}=\zeta_{3}, A_{22}=\omega_{1}, t_{4-n}=-6 \lambda_{2}, \tilde{A}_{44}=\phi_{4}-\frac{1}{3}\left(18 \zeta_{3} \lambda_{2}+\omega_{1}^{2}\right)$, $A_{4 j}=f_{j}(5 \leq j \leq 8), \tilde{A}_{68}=g_{8}+\frac{1}{3} \zeta_{3} f_{7}+\frac{1}{12} \tau^{2} f_{8}, \tilde{A}_{69}=g_{9}+\frac{1}{3} \zeta_{3} f_{8}, \tilde{A}_{6,10}=g_{10}$ and $A_{6 j}=g_{j}(j=11,12)$.

${ }^{21}$ Unlike $\mathrm{CY}_{3}^{(1)}$, it seems that $\mathrm{CY}_{3}^{(2)}$ cannot be unHiggsed to any $\mathrm{CY}_{3}$ with $G=\mathrm{SU}(8)$ (see the next subsection).
} 
Among them, we find that setting

$$
s_{4}=0
$$

yields a $\mathrm{CY}_{3}$ with $G=\mathrm{SU}(6) \times \mathrm{SU}(2)$. We then map $f$ and $g$ to $f^{\prime}$ and $g^{\prime}$, and set the polynomials (6.13) contained in $f^{\prime}$ and $g^{\prime}$ to zero. It gives a RES-fibered geometry with $G=$ $\mathrm{SU}(6) \times \mathrm{SU}(2)$. The explicit forms of $f_{\text {res }}, g_{\text {res }}$ and $\Delta_{\text {res }}$ are given in appendix C.7. In this way, we obtain the geometry for the non-Cartan Mordell-Weil lattice $E(K)=A_{1}^{*} \oplus\langle 1 / 6\rangle$ in table 1.

Among the independent polynomials (7.6), we have required $s_{4}=0$, and hence the number of singlets is reduced by $4+1=5$ from (7.7),

$$
n\left(H_{0}\right)=2 n+1-5=2 n-4 .
$$

The charged matter spectrum is derived in appendix C.7 and given in (C.31). Together with the singlets (7.10), the full spectrum is given by

$$
\begin{aligned}
2 n-4 & :(\mathbf{1}, \mathbf{1}) \\
n+12 & :(\mathbf{6}, \mathbf{1}) \\
6 & :(\mathbf{1 5}, \mathbf{1}) \\
n-4: & \frac{1}{2}(\mathbf{2 0}, \mathbf{1}) \\
16 & :(\mathbf{1}, \mathbf{2}) \\
n & :(\mathbf{6}, \mathbf{2})
\end{aligned}
$$

This F-theoretic spectrum is equivalent to the heterotic spectrum (see appendix B.1) given in table 18 with $r=12$.

\subsection{Other cases}

We expect that the other tunings give the other models in the $\mathrm{SU}(8)$ series, but we have not obtained corresponding RES-fibered geometries yet. For example, setting

$$
h_{n}^{\prime}=r_{n-4} s_{4}
$$

is expected to give a $\mathrm{CY}_{3}$ with $G=\mathrm{SU}(4) \times \mathrm{SU}(4)$, but it leads to unresolvable singularities. For the enhancement $\mathrm{SU}(3) \times \mathrm{SU}(2) \times \mathrm{SU}(2) \rightarrow \mathrm{SU}(3) \times \mathrm{SU}(4)$ discussed in section 6.3 , we were able to deform such singularities to resolvable ones. In the present case, however, we could not find how to deform them suitably. Since we have not obtained a $\mathrm{CY}_{3}$ yet, we do not have a RES-fibered geometry, either. There are two possibilities. First, there are such geometries but we just haven't found them yet. Second, one can prove that there are no such geometries in these cases. ${ }^{22}$ It would be interesting to find which is correct, but we leave it for future work. Also, we could not find the way of realizing the enhancement $\mathrm{SU}(3) \times \mathrm{SU}(2) \times \mathrm{SU}(2) \rightarrow \mathrm{SU}(3) \times \mathrm{SU}(3) \times \mathrm{SU}(2)$. We also leave this issue for future investigations.

\footnotetext{
${ }^{22}$ The argument given in [74] may be valid to analyze these cases.
} 


\section{Conclusions}

In this paper, we studied how the non-Cartan Mordell-Weil lattices are realized in the sixdimensional heterotic/F-theory duality. We focused on the SU(7) series and SU(8) series given in table 1.

On the heterotic string side, we give a procedure to derive the massless matter spectrum by using index theorem. Except No.45, the gauge bundle $H$ is a product of a semi-simple group and one or more $\mathrm{U}(1)$ group $(\mathrm{s})$. The total $12+n$ instantons are distributed into each group factor. We found that the U(1) instanton numbers are a multiple of some particular non-one integer $r$ and the resulting number of each matter is integral. We showed that this integer $r$ is determined by the lattice spacing of the $\mathrm{U}(1)$ direction in the $E_{8}$ lattice, which is orthogonal to the lattice of the semi-simple part of $H$ and the singularity lattice $T$. For $\operatorname{rank}(E(K))>2$ cases, more than one choices of the number of $\mathrm{U}(1)$ direction(s) in $H$ are possible for the given MW lattice. In these cases, more than one matter spectra are obtained for a gauge symmetry $G$. We found that if the number of the $U(1)$ factor(s) is larger, the spectrum is finer.

On the dual F-theory side, we examined how to construct a geometry for a given non-Cartan MW lattice $E(K)$. We first construct $\mathrm{CY}_{3}$ with singularity $G$ of the lattice $T$ for the given $E(K)$. It starts from $G=\mathrm{SU}(2) \times \mathrm{SU}(2) \times \mathrm{SU}(2)$ case, and successive unHiggsing processes are carefully performed. Then we construct the map from these $\mathrm{CY}_{3}$ to RES-fibered threefolds. In order that the singularity $G$ is unchanged by the map, we generically have to do slight modifications on the geometry. As a result, we obtained the explicit forms of RES-fibered geometries for all the $\mathrm{SU}(7)$ series and for some cases in the $\mathrm{SU}(8)$ series $(G=\mathrm{SU}(4) \times \mathrm{SU}(2) \times \mathrm{SU}(2)($ No.22) and $G=\mathrm{SU}(6) \times \mathrm{SU}(2)($ No.29)). Note that the geometries for the $\mathrm{SU}(8)$ series we have constructed cannot be obtained from the (Higgsing of the) known $\mathrm{CY}_{3}$ with $G=\mathrm{SU}(8)$ of [66], because mapping that $\mathrm{CY}_{3}$ to a RES-fibered geometry inevitably changes the singularity. We found that the F-theoretic spectrum derived from each of these geometries is completely identical to the heterotic spectrum with a specific distribution of instantons.

We have not succeeded to construct $\mathrm{CY}_{3}$ 's (and hence RES-fibered threefolds) for $\mathrm{SU}(8)$ series other than the above two cases yet. It would be an interesting problem to understand whether there are no such geometries in principle, or we merely have not found them yet for some technical reason; we leave it for future investigations. Also, each RESfibered geometry for $\mathrm{SU}(7)$ and $\mathrm{SU}(8)$ series we have constructed in this paper corresponds only to a particular distribution of instantons. It is expected that the deformation to the generic distributions can be done similarly to the case of $G=\mathrm{SU}(2) \times \mathrm{SU}(2) \times \mathrm{SU}(2)$, though it is technically much more elaborated. We also leave this issue for future work.

\section{Acknowledgments}

We thank K. Hori, Y. Kimura, Y. Yamada and T. Watari for discussions. T. T. would also like to thank Kavli Institute for the Physics and Mathematics of the Universe (Kavli IPMU) for hospitality, where most of his work was done. The work of S. M. is supported 
by Grant-in-Aid for Scientific Research (C) \#16K05337 from The Ministry of Education, Culture, Sports, Science and Technology of Japan.

\section{A Heterotic index computations for the $\mathrm{SU}(7)$ series}

$$
\text { A.1 } G=\mathrm{SU}(4) \times \mathrm{SU}(3)\left(T=A_{3} \oplus A_{2}\right)(\text { No.19) }
$$

\section{A.1.1 The case when $H=\mathrm{SU}(2) \times \mathrm{U}(1) \times \mathrm{U}(1)$}

The junction lattice is

$$
\Lambda_{(19)}^{-1}=\left(\begin{array}{crr}
2 & 0 & -1 \\
0 & 2 & -1 \\
-1 & -1 & 4
\end{array}\right) .
$$

As we did in the case No.17 in section 3.4, we can break $\mathrm{SU}(7)$ to $\mathrm{SU}(4) \times \mathrm{SU}(3)$ by further introducing instantons in another $\mathrm{U}(1)(=\widetilde{\widetilde{U}}(1))$. We assume $12+n-r-\tilde{\tilde{r}}, r$ and $\tilde{\tilde{r}}$ instantons for each factor of $H=\mathrm{SU}(2) \times \mathrm{U}(1) \times \widetilde{\widetilde{U}}(1)$.

The decomposition of $\mathbf{2 4 8}$ is as shown in table 11. In this case

$$
\begin{aligned}
& \sum_{E_{8}}(\mathrm{U}(1) \text { charge })^{2}=14 \cdot 60, \\
& \sum_{E_{8}}(\tilde{\tilde{U}}(1) \text { charge })^{2}=84 \cdot 60 .
\end{aligned}
$$

Therefore

$$
\begin{aligned}
\frac{1}{8 \pi^{2}} \int F^{a} \wedge F^{b} \operatorname{Tr} \tau^{a} \tau^{b}=12+n-r-\tilde{\tilde{r}} \\
\frac{1}{8 \pi^{2}} \int F^{\mathrm{U}(1)} \wedge F^{\mathrm{U}(1)}=\frac{r}{14} \\
\frac{1}{8 \pi^{2}} \int F^{\widetilde{\widetilde{U}}(1)} \wedge F^{\widetilde{\widetilde{U}}(1)}=\frac{\tilde{\tilde{r}}}{84} .
\end{aligned}
$$

The computed spectrum is shown in table 12 . The multiplicity of $(\mathbf{4 , 3})$ is a sum of

$$
n_{(\mathbf{4}, \mathbf{3})}+n_{(\overline{\mathbf{4}}, \overline{\mathbf{3}})}=-4+\frac{2}{7} r+\frac{37}{42} \tilde{\tilde{r}}
$$

and

$$
n_{(\overline{\mathbf{4}}, \mathbf{3})}+n_{(\mathbf{4}, \overline{\mathbf{3}})}=n+8-\frac{6}{7} r-\frac{41}{42} \tilde{\tilde{r}} .
$$

The general condition for $\frac{2}{7} r+\frac{1}{21} \tilde{\tilde{r}}$ to be an integer is

$$
(r, \tilde{\tilde{r}})=(3,3) k+(-1,6) l \quad(k, l \in \mathbb{Z}),
$$

and then

$$
\frac{2}{7} r+\frac{1}{21} \tilde{\tilde{r}}=k \in \mathbb{Z} .
$$




\begin{tabular}{|c|c|c|c|}
\hline Rep. of $\mathrm{SU}(4) \times \mathrm{SU}(3)$ & Rep. of SU(2) & $\frac{2}{3} h$ charge & $\tilde{\tilde{h}}$ charge \\
\hline$(15,1)$ & 1 & 0 & 0 \\
\hline$(1,8)$ & 1 & 0 & 0 \\
\hline$(4, \overline{\mathbf{3}})$ & 1 & 0 & 7 \\
\hline$(\overline{4}, 3)$ & 1 & 0 & -7 \\
\hline$(\mathbf{1}, \mathbf{1})(\tilde{\tilde{h}})$ & 1 & 0 & 0 \\
\hline$(4,1)$ & 2 & 3 & 3 \\
\hline$(4,1)$ & 1 & -4 & 3 \\
\hline$(\mathbf{1}, \mathbf{3})$ & 2 & 3 & -4 \\
\hline$(\mathbf{1}, \mathbf{3})$ & 1 & -4 & -4 \\
\hline$(\overline{4}, \mathbf{1})$ & 2 & -3 & -3 \\
\hline$(\overline{4}, \mathbf{1})$ & 1 & 4 & -3 \\
\hline$(\mathbf{1}, \overline{\mathbf{3}})$ & 2 & -3 & 4 \\
\hline$(\mathbf{1}, \overline{\mathbf{3}})$ & 1 & 4 & 4 \\
\hline$(\overline{4}, \mathbf{1})$ & 1 & 2 & 9 \\
\hline$(6,3)$ & 1 & 2 & 2 \\
\hline$(4, \overline{3})$ & 1 & 2 & -5 \\
\hline$(\mathbf{1}, \mathbf{1})$ & 1 & 2 & -12 \\
\hline$(4,1)$ & 1 & -2 & -9 \\
\hline$(\mathbf{6}, \overline{\mathbf{3}})$ & 1 & -2 & -2 \\
\hline$(\overline{4}, \mathbf{3})$ & 1 & -2 & 5 \\
\hline$(\mathbf{1}, \mathbf{1})$ & 1 & -2 & 12 \\
\hline$(6,1)$ & 2 & -1 & 6 \\
\hline$(4,3)$ & 2 & -1 & -1 \\
\hline$(\mathbf{1}, \overline{\mathbf{3}})$ & 2 & -1 & -8 \\
\hline$(\mathbf{6}, \mathbf{1})$ & 2 & 1 & -6 \\
\hline$(\overline{4}, \overline{\mathbf{3}})$ & 2 & 1 & 1 \\
\hline$(\mathbf{1}, \mathbf{3})$ & 2 & 1 & 8 \\
\hline$(\mathbf{1}, \mathbf{1})\left(\frac{2}{3} h\right)$ & 1 & 0 & 0 \\
\hline$(\mathbf{1}, \mathbf{1})$ & 3 & 0 & 0 \\
\hline
\end{tabular}

Table 11. Decomposition of $\mathbf{2 4 8}$. 


\begin{tabular}{|c|c|}
\hline Representation & Multiplicity \\
\hline$(\mathbf{1 5}, \mathbf{1})$ & $-1(\mathrm{SU}(4)$ vector $)$ \\
$(\mathbf{1}, \mathbf{8})$ & $-1(\mathrm{SU}(3)$ vector $)$ \\
$(\mathbf{1}, \mathbf{1})$ & $2 n+17-6\left(\frac{2}{7} r+\frac{1}{21} \tilde{\tilde{r}}\right)$ \\
$(\mathbf{4}, \mathbf{3})$ & $n+4-2\left(\frac{2}{7} r+\frac{1}{21} \tilde{\tilde{r}}\right)$ \\
$(\mathbf{4}, \mathbf{1})$ & $n+4+6\left(\frac{2}{7} r+\frac{1}{21} \tilde{\tilde{r}}\right)$ \\
$(\mathbf{1}, \mathbf{3})$ & $2 n+14+2\left(\frac{2}{7} r+\frac{1}{21} \tilde{\tilde{r}}\right)$ \\
$(\mathbf{6}, \mathbf{3})$ & $-2+\left(\frac{2}{7} r+\frac{1}{21} \tilde{\tilde{r}}\right)$ \\
$(\mathbf{6}, \mathbf{1})$ & $n+8-3\left(\frac{2}{7} r+\frac{1}{21} \tilde{\tilde{r}}\right)$ \\
\hline
\end{tabular}

Table 12. The spectrum for the configuration No.19.

The orthogonal decomposition of the junction lattice (inverse the MW lattice) is

$$
\left(\begin{array}{lll}
7 & 1 & 2 \\
0 & 1 & 0 \\
0 & 0 & 1
\end{array}\right)\left(\begin{array}{crr}
2 & 0 & -1 \\
0 & 2 & -1 \\
-1 & -1 & 4
\end{array}\right)\left(\begin{array}{lll}
7 & 0 & 0 \\
1 & 1 & 0 \\
2 & 0 & 1
\end{array}\right)=\left(\begin{array}{ccc}
84 & 0 & 0 \\
0 & 2 & -1 \\
0 & -1 & 4
\end{array}\right) .
$$

The right-bottom block is $\Lambda_{(25)}^{-1}$.

\section{A.1.2 The case when $H=\mathrm{SU}(2) \times \mathrm{SU}(2) \times \mathrm{U}(1)$}

$\Lambda_{(19)}^{-1}$ can also be regarded as an extension of the semi-simple $\mathrm{SU}(2) \times \mathrm{SU}(2)$ Cartan matrix. $E_{8}$ is decomposed into representations of $\mathrm{SU}(6) \times \mathrm{SU}(3) \times \mathrm{SU}(2)$ as

$$
\begin{aligned}
\mathbf{2 4 8}= & (\mathbf{3 5}, \mathbf{1}, \mathbf{1}) \oplus(\mathbf{1}, \mathbf{8}, \mathbf{1}) \oplus(\mathbf{1}, \mathbf{1}, \mathbf{3}) \\
& \oplus(\mathbf{1 5}, \mathbf{3}, \mathbf{1}) \oplus(\overline{\mathbf{1 5}}, \overline{\mathbf{3}}, \mathbf{1}) \oplus(\mathbf{2 0}, \mathbf{1}, \mathbf{2}) \oplus(\mathbf{6}, \overline{\mathbf{3}}, \mathbf{2}) \oplus(\mathbf{6}, \mathbf{3}, \mathbf{2}) .
\end{aligned}
$$

By further breaking this $\mathrm{SU}(6)$ into $\mathrm{SU}(4) \times \mathrm{SU}(2)\left(\equiv \mathrm{SU}(2)^{\prime}\right)$ by an $\mathrm{U}(1)\left(\equiv \mathrm{U}(1)_{(19)}\right)$ one obtains the decomposition in representations of $\mathrm{SU}(4) \times \mathrm{SU}(3) \times \mathrm{SU}(2) \times \mathrm{SU}(2)^{\prime} \times \mathrm{U}(1)_{(19)}$ (table 13). The instanton numbers are assumed to be $12+n-r-r_{\mathrm{U}(1)}, r$ and $r_{\mathrm{U}(1)}$ for $\mathrm{SU}(2), \mathrm{SU}(2)^{\prime}$ and $\mathrm{U}(1)_{(19)}$. The sum of $\left(\mathrm{U}(1)_{(19)} \text { charge }\right)^{2}$ is

$$
\sum_{E_{8}}\left(\mathrm{U}(1)_{(19)} \text { charge }\right)^{2}=12 \cdot 60
$$

so that

$$
\begin{aligned}
\frac{1}{8 \pi^{2}} \int F^{a} \wedge F^{b} \operatorname{Tr} \tau^{a} \tau^{b} & =12+n-r-r_{\mathrm{U}(1)}, \\
\frac{1}{8 \pi^{2}} \int F^{\prime a} \wedge F^{\prime b} \operatorname{Tr} \tau^{\prime a} \tau^{\prime b} & =r \\
\frac{1}{8 \pi^{2}} \int F^{\mathrm{U}(1)} \wedge F^{\mathrm{U}(1)} & =\frac{r_{\mathrm{U}(1)}}{12} .
\end{aligned}
$$




\begin{tabular}{|ccccc|}
\hline Rep. of SU(4) & Rep. of SU(3) & Rep. of SU(2) & Rep. of SU $(2)^{\prime}$ & $\left.\mathrm{U}^{(1)}\right)_{(19)}$ charge \\
\hline $\mathbf{1 5}$ & $\mathbf{1}$ & $\mathbf{1}$ & $\mathbf{1}$ & 0 \\
$\mathbf{1}$ & $\mathbf{1}$ & $\mathbf{1}$ & $\mathbf{1}$ & 0 \\
$\mathbf{1}$ & $\mathbf{1}$ & $\mathbf{1}$ & $\mathbf{3}$ & 0 \\
$\mathbf{4}$ & $\mathbf{1}$ & $\mathbf{1}$ & $\mathbf{2}$ & 3 \\
$\overline{\mathbf{4}}$ & $\mathbf{1}$ & $\mathbf{1}$ & $\mathbf{2}$ & -3 \\
$\overline{\mathbf{4}}$ & $\mathbf{1}$ & $\mathbf{2}$ & $\mathbf{1}$ & 3 \\
$\mathbf{6}$ & $\mathbf{1}$ & $\mathbf{2}$ & $\mathbf{2}$ & 0 \\
$\mathbf{4}$ & $\mathbf{1}$ & $\mathbf{2}$ & $\mathbf{1}$ & -3 \\
$\mathbf{6}$ & $\mathbf{3}$ & $\mathbf{1}$ & $\mathbf{1}$ & 2 \\
$\mathbf{4}$ & $\mathbf{3}$ & $\mathbf{1}$ & $\mathbf{2}$ & -1 \\
$\mathbf{1}$ & $\mathbf{3}$ & $\mathbf{1}$ & $\mathbf{1}$ & -4 \\
$\mathbf{6}$ & $\overline{\mathbf{3}}$ & $\mathbf{1}$ & $\mathbf{1}$ & -2 \\
$\overline{\mathbf{4}}$ & $\overline{\mathbf{3}}$ & $\mathbf{1}$ & $\mathbf{2}$ & 1 \\
$\mathbf{1}$ & $\overline{\mathbf{3}}$ & $\mathbf{1}$ & $\mathbf{1}$ & 4 \\
$\mathbf{4}$ & $\overline{\mathbf{3}}$ & $\mathbf{2}$ & $\mathbf{1}$ & 1 \\
$\mathbf{1}$ & $\overline{\mathbf{3}}$ & $\mathbf{2}$ & $\mathbf{2}$ & -2 \\
$\overline{\mathbf{4}}$ & $\mathbf{3}$ & $\mathbf{2}$ & $\mathbf{1}$ & -1 \\
$\mathbf{1}$ & $\mathbf{3}$ & $\mathbf{2}$ & $\mathbf{2}$ & 2 \\
$\mathbf{1}$ & $\mathbf{8}$ & $\mathbf{1}$ & $\mathbf{1}$ & 0 \\
$\mathbf{1}$ & $\mathbf{1}$ & $\mathbf{3}$ & $\mathbf{1}$ & 0 \\
\hline
\end{tabular}

Table 13. Decomposition of $\mathbf{2 4 8}$ in reps. of $\mathrm{SU}(4) \times \mathrm{SU}(3) \times \mathrm{SU}(2) \times \mathrm{SU}(2)^{\prime} \times \mathrm{U}(1)$.

\begin{tabular}{|c|c|}
\hline Representation & Multiplicity \\
\hline$(\mathbf{1 5}, \mathbf{1})$ & $-1(\mathrm{SU}(4)$ vector $)$ \\
$(\mathbf{1}, \mathbf{8})$ & $-1(\mathrm{SU}(3)$ vector $)$ \\
$(\mathbf{1}, \mathbf{1})$ & $2 n+17-12 r_{\mathrm{U}(1)}^{\prime}$ \\
$(\mathbf{4}, \mathbf{3})$ & $-4+r+r_{\mathrm{U}(1)}^{\prime}$ \\
$(\mathbf{4}, \overline{\mathbf{3}})$ & $n+8-r-5 r_{\mathrm{U}(1)}^{\prime}$ \\
$(\mathbf{4}, \mathbf{1})$ & $n+4+12 r_{\mathrm{U}(1)}^{\prime}$ \\
$(\mathbf{1}, \mathbf{3})$ & $2 n+14+4 r_{\mathrm{U}(1)}^{\prime}$ \\
$(\mathbf{6}, \mathbf{3})$ & $-2+2 r_{\mathrm{U}(1)}^{\prime}$ \\
$(\mathbf{6}, \mathbf{1})$ & $n+8-6 r_{\mathrm{U}(1)}^{\prime}$ \\
\hline
\end{tabular}

Table 14. The spectrum for the configuration No.19. $H=\mathrm{SU}(2) \times \mathrm{SU}(2) \times \mathrm{U}(1)$. 
The spectrum in this case is summarized in table 14, where

$$
r_{\mathrm{U}(1)}=6 r_{\mathrm{U}(1)}^{\prime}
$$

This coincides with the result shown in table 12 if $(\mathbf{4}, \mathbf{3})$ and $(\mathbf{4}, \overline{\mathbf{3}})$ are identified and the replacement

$$
\frac{2}{7} r+\frac{1}{21} \tilde{\tilde{r}}=k=2 r_{\mathrm{U}(1)}^{\prime}
$$

is made. Again, the spectrum for $\mathrm{SU}(2) \times \mathrm{U}(1) \times \mathrm{U}(1)$ is more general than that for $\mathrm{SU}(2) \times \mathrm{SU}(2) \times \mathrm{U}(1)$ as $k$ is restricted to even in the latter. The orthogonal decomposition of the junction lattice in this case is

$$
\left(\begin{array}{lll}
2 & 0 & 0 \\
0 & 2 & 0 \\
1 & 1 & 2
\end{array}\right)\left(\begin{array}{rrr}
2 & 0 & -1 \\
0 & 2 & -1 \\
-1 & -1 & 4
\end{array}\right)\left(\begin{array}{lll}
2 & 0 & 1 \\
0 & 2 & 1 \\
0 & 0 & 2
\end{array}\right)=\left(\begin{array}{llr}
2 & 0 & 0 \\
0 & 2 & 0 \\
0 & 0 & 12
\end{array}\right),
$$

this agrees with the fact that the $\mathrm{U}(1)$ instanton number is a multiple of 6 .

\section{$\mathrm{A} .2 \quad G=\mathrm{SU}(3) \times \mathrm{SU}(2) \times \mathrm{SU}(2)\left(T=A_{2} \oplus A_{1} \oplus A_{1}\right)($ No.12)}

The inverse of the MW lattice is

$$
\Lambda_{(12)}^{-1}=\left(\begin{array}{cccc}
4 & -1 & 0 & 1 \\
-1 & 2 & -1 & 0 \\
0 & -1 & 2 & -1 \\
1 & 0 & -1 & 2
\end{array}\right)
$$

It has the $\mathrm{SU}(4)$ Cartan matrix in the right-bottom block, so we first take $\mathrm{SU}(4) \times \mathrm{U}(1)$ as $H$ and compute the spectrum. Since $G=\mathrm{SU}(3) \times \mathrm{SU}(2) \times \mathrm{SU}(2)$, we can use the decomposition table 13 for No.19. Assuming the instanton distribution $12+n-r_{\mathrm{U}(1)}, r_{\mathrm{U}(1)}$ for $\mathrm{SU}(4), \mathrm{U}(1)$, respectively, we find the spectrum as shown in table $15\left(r_{\mathrm{U}(1)}=3 r_{\mathrm{U}(1)}^{\prime}\right)$.

Orthogonal decomposition of the inverse of the MW lattice:

$$
\left(\begin{array}{cccc}
2 & 1 & 0 & -1 \\
0 & 1 & 0 & 0 \\
0 & 0 & 1 & 0 \\
0 & 0 & 0 & 1
\end{array}\right)\left(\begin{array}{cccc}
4 & -1 & 0 & 1 \\
-1 & 2 & -1 & 0 \\
0 & -1 & 2 & -1 \\
1 & 0 & -1 & 2
\end{array}\right)\left(\begin{array}{cccc}
2 & 0 & 0 & 0 \\
1 & 1 & 0 & 0 \\
0 & 0 & 1 & 0 \\
-1 & 0 & 0 & 1
\end{array}\right)=\left(\begin{array}{cccc}
12 & 0 & 0 & 0 \\
0 & 2 & -1 & 0 \\
0 & -1 & 2 & -1 \\
0 & 0 & -1 & 2
\end{array}\right) \text {. }
$$

This spectrum can also be computed by breaking $\mathrm{SU}(5) \times \mathrm{SU}(2)$ for No.17 to $\mathrm{SU}(3) \times$ $\mathrm{SU}(2) \times \mathrm{SU}(2)$ by giving instantons to $\mathrm{U}(1)_{(17)}$. In this case the multiplicities can be simply obtained by adding

$$
\frac{r_{(17)}}{30} \cdot\left(\mathrm{U}(1)_{(17)} \text { charge }\right)^{2} \cdot(\text { the dimension of the representation of } H(2 \text { or } 1))
$$

for each $\mathrm{SU}(3) \times \mathrm{SU}(2)$ representation in the $\mathrm{SU}(5)$ decomposition in No.17. Assuming the instanton numbers to be $12+n-r-\tilde{r}-r_{(17)}, r, \tilde{r}$ and $r_{(17)}$ for $\mathrm{SU}(2), \mathrm{U}(1) \widetilde{\mathrm{U}(1)}$ 


\begin{tabular}{|c|c|}
\hline Representation & Multiplicity \\
\hline$(\mathbf{8}, \mathbf{1}, \mathbf{1})$ & $-1(\mathrm{SU}(3)$ vector $)$ \\
$(\mathbf{1}, \mathbf{3}, \mathbf{1})$ & $-1(\mathrm{SU}(2)$ vector $)$ \\
$(\mathbf{1}, \mathbf{1}, \mathbf{3})$ & $-1\left(\mathrm{SU}(2)^{\prime}\right.$ vector $)$ \\
$(\mathbf{1}, \mathbf{1}, \mathbf{1})$ & $4 n+32-12 r_{\mathrm{U}(1)}^{\prime}$ \\
$(\mathbf{1}, \mathbf{1}, \mathbf{2})$ & $n+4+6 r_{\mathrm{U}(1)}^{\prime}$ \\
$(\mathbf{1}, \mathbf{2}, \mathbf{1})$ & $n+4+6 r_{\mathrm{U}(1)}^{\prime}$ \\
$(\mathbf{1}, \mathbf{2}, \mathbf{2})$ & $n+6-3 r_{\mathrm{U}(1)}^{\prime}$ \\
$(\mathbf{3}, \mathbf{1}, \mathbf{1})$ & $2 n+10+4 r_{\mathrm{U}(1)}^{\prime}$ \\
$(\mathbf{3}, \mathbf{1}, \mathbf{2})$ & $n+4-2 r_{\mathrm{U}(1)}^{\prime}$ \\
$(\mathbf{3}, \mathbf{2}, \mathbf{1})$ & $n+4-2 r_{\mathrm{U}(1)}^{\prime}$ \\
$(\mathbf{3}, \mathbf{2}, \mathbf{2})$ & $-2+r_{\mathrm{U}(1)}^{\prime}$ \\
\hline
\end{tabular}

Table 15. The spectrum for the configuration No.12. $H=\mathrm{SU}(4) \times \mathrm{U}(1)$.

\begin{tabular}{|c|c|}
\hline Representation & Multiplicity \\
\hline$(\mathbf{8}, \mathbf{1}, \mathbf{1})$ & $-1(\mathrm{SU}(3)$ vector $)$ \\
$(\mathbf{1}, \mathbf{3}, \mathbf{1})$ & $-1(\mathrm{SU}(2)$ vector $)$ \\
$(\mathbf{1}, \mathbf{1}, \mathbf{3})$ & $-1\left(\mathrm{SU}(2)^{\prime}\right.$ vector $)$ \\
$(\mathbf{1}, \mathbf{1}, \mathbf{1})$ & $4 n+32-12\left(\frac{2}{7} r+\frac{1}{70} \tilde{r}+\frac{1}{30} r_{(17)}\right)$ \\
$(\mathbf{1}, \mathbf{1}, \mathbf{2})$ & $n+4+6\left(\frac{2}{7} r+\frac{1}{70} \tilde{r}+\frac{1}{30} r_{(17)}\right)$ \\
$(\mathbf{1}, \mathbf{2}, \mathbf{1})$ & $n+4+6\left(\frac{2}{7} r+\frac{1}{70} \tilde{r}+\frac{1}{30} r_{(17)}\right)$ \\
$(\mathbf{1}, \mathbf{2}, \mathbf{2})$ & $n+6-3\left(\frac{2}{7} r+\frac{1}{70} \tilde{r}+\frac{1}{30} r_{(17)}\right)$ \\
$(\mathbf{3}, \mathbf{1}, \mathbf{1})$ & $2 n+10+4\left(\frac{2}{7} r+\frac{1}{70} \tilde{r}+\frac{1}{30} r_{(17)}\right)$ \\
$(\mathbf{3}, \mathbf{1}, \mathbf{2})$ & $n+4-2\left(\frac{2}{7} r+\frac{1}{70} \tilde{r}+\frac{1}{30} r_{(17)}\right)$ \\
$(\mathbf{3}, \mathbf{2}, \mathbf{1})$ & $n+4-2\left(\frac{2}{7} r+\frac{1}{70} \tilde{r}+\frac{1}{30} r_{(17)}\right)$ \\
$(\mathbf{3}, \mathbf{2}, \mathbf{2})$ & $-2+\left(\frac{2}{7} r+\frac{1}{70} \tilde{r}+\frac{1}{30} r_{(17)}\right)$ \\
\hline
\end{tabular}

Table 16. The spectrum for the configuration No.12. $H=\mathrm{SU}(2) \times \mathrm{U}(1)^{3}$.

and $\mathrm{U}(1)_{(17)}$, respectively, we obtain the result as shown in table 16 , which coincides with table 15 provided that

$$
r_{\mathrm{U}(1)}^{\prime}=\frac{2}{7} r+\frac{1}{70} \tilde{r}+\frac{1}{30} r_{(17)} .
$$

For $\frac{2}{7} r+\frac{1}{70} \tilde{r}+\frac{1}{30} r_{(17)}$ to be integer, the general solution is

$$
\left(r, \tilde{r}, r_{(17)}\right)=(3,3,3) k+(-1,20,0) l+(0,-7,3) m \quad(k, l, m \in \mathbb{Z}),
$$


in which

$$
\frac{2}{7} r+\frac{1}{70} \tilde{r}+\frac{1}{30} r_{(17)}=k \in \mathbb{Z}
$$

\section{B Heterotic index computations for the $\mathrm{SU}(8)$ series}

\section{B.1 $G=\mathrm{SU}(6) \times \mathrm{SU}(2)\left(T=A_{5} \oplus A_{1}\right)(\mathrm{No} .29)$}

There are two $A_{7}=\mathrm{SU}(8)$ embeddings in the $E_{8}$ root lattice, No.45 and No.44. The former $\mathrm{SU}(8)$ is the one in the $\mathrm{SU}(9)$ sublattice of $E_{8}$, while the latter is the one in the $E_{7}$ sublattice. Correspondingly there are two $G=A_{5} \oplus A_{1}(\mathrm{SU}(6) \times \mathrm{SU}(2))$ cases, No.29 and No.28. The present case No.29 is the one descended from No.45.

In this case $E(K)=A_{1}^{*} \oplus\langle 1 / 6\rangle$, and thus $H=\mathrm{SU}(2) \times \mathrm{U}(1)$. Taking

$$
\mathbf{e}_{1}-\mathbf{e}_{2}, \mathbf{e}_{2}-\mathbf{e}_{3}, \mathbf{e}_{3}-\mathbf{e}_{4}, \mathbf{e}_{4}-\mathbf{e}_{5}, \mathbf{e}_{5}-\mathbf{e}_{6}
$$

and

$$
\mathbf{e}_{7}-\mathbf{e}_{8}
$$

as the simple roots of $G=\mathrm{SU}(6) \times \mathrm{SU}(2)$ in the notation (3.13)(3.14), the simple root of the $\mathrm{SU}(2)$ in $H$ is

$$
\mathbf{e}_{7}+\mathbf{e}_{8}+\mathbf{e}_{9}-\frac{1}{3} \sum_{l=1}^{9} \mathbf{e}_{l} \equiv \mathbf{e}_{789},
$$

whereas the $E_{8}$ root corresponding to the $\mathrm{U}(1)$ in $H\left(\equiv \mathrm{U}(1)_{\langle 1 / 6\rangle}\right)$ is

$$
\mathbf{e}_{7}+\mathbf{e}_{8}-2 \mathbf{e}_{9} \equiv \boldsymbol{\omega}_{\langle 1 / 6\rangle}
$$

Indeed, we have $\mathbf{e}_{789}^{2}=2, \boldsymbol{\omega}_{\langle 1 / 6\rangle}^{2}=6$ and $\mathbf{e}_{789} \cdot \boldsymbol{\omega}_{\langle 1 / 6\rangle}=0$. The decomposition of $E_{8}$ in this $G \times H$ is as shown in table 17 .

Assuming the instantons in $12+n-r$ and $r$ in $\mathrm{SU}(2)$ and $\mathrm{U}(1)_{\langle 1 / 6\rangle}$, we obtain the spectrum of No.29 as shown in table 18.

\section{B.2 $G=\mathrm{SU}(4) \times \mathrm{SU}(2) \times \mathrm{SU}(2)\left(T=A_{3} \oplus A_{1} \oplus A_{1}\right)(\mathrm{No.22})$}

This is one of the two $G=\mathrm{SU}(4) \times \mathrm{SU}(2) \times \mathrm{SU}(2)$ cases, the one descended from No.29. $E(K)=A_{1}^{*} \oplus A_{1}^{*} \oplus\langle 1 / 4\rangle$, and thus $H=\mathrm{SU}(2) \times \mathrm{SU}(2) \times \mathrm{U}(1)$. Taking

$$
\mathbf{e}_{1}-\mathbf{e}_{2}, \mathbf{e}_{2}-\mathbf{e}_{3}, \mathbf{e}_{3}-\mathbf{e}_{4}, \mathbf{e}_{4}-\mathbf{e}_{5}
$$

as the simple roots of $\mathrm{SU}(4) \subset G$ and

$$
\mathbf{e}_{5}-\mathbf{e}_{6}, \mathbf{e}_{7}-\mathbf{e}_{8}
$$

as those of $\mathrm{SU}(2) \times \mathrm{SU}(2)\left(\equiv \mathrm{SU}(2)_{56} \times \mathrm{SU}(2)_{78}\right) \subset G$, the simple roots of $\mathrm{SU}(2) \times \mathrm{SU}(2) \subset H$ $\left(\equiv \mathrm{SU}(2)_{569} \times \mathrm{SU}(2)_{789}\right)$ are

$$
\begin{gathered}
\mathbf{e}_{5}+\mathbf{e}_{6}+\mathbf{e}_{9}-\frac{1}{3} \sum_{l=1}^{9} \mathbf{e}_{l}, \\
\mathbf{e}_{7}+\mathbf{e}_{8}+\mathbf{e}_{9}-\frac{1}{3} \sum_{l=1}^{9} \mathbf{e}_{l},
\end{gathered}
$$




\begin{tabular}{|c|cc|}
\hline Rep. of $\mathrm{SU}(6) \times \mathrm{SU}(2)$ & Rep. of $\mathrm{SU}(2) \subset H$ & $\mathrm{U}(1)_{\langle 1 / 6\rangle}$ charge \\
\hline$(\mathbf{3 5}, \mathbf{1})(\mathrm{SU}(6))$ & $\mathbf{1}$ & 0 \\
$(\mathbf{6}, \mathbf{2})$ & $\mathbf{2}$ & -1 \\
$(\overline{\mathbf{6}}, \mathbf{2})$ & $\mathbf{2}$ & 1 \\
$(\mathbf{1}, \mathbf{3})(\mathrm{SU}(2))$ & $\mathbf{1}$ & 0 \\
$(\mathbf{6}, \mathbf{1})$ & $\mathbf{2}$ & 2 \\
$(\overline{\mathbf{6}}, \mathbf{1})$ & $\mathbf{2}$ & -2 \\
$(\mathbf{1}, \mathbf{2})$ & $\mathbf{1}$ & 3 \\
$(\mathbf{1}, \mathbf{2})$ & $\mathbf{1}$ & -3 \\
$(\mathbf{2 0}, \mathbf{1})$ & & 0 \\
$(\mathbf{1 5}, \mathbf{2})$ & $\mathbf{2}$ & 1 \\
$(\overline{\mathbf{1 5}}, \mathbf{2})$ & $\mathbf{1}$ & -1 \\
$(\mathbf{1 5}, \mathbf{1})$ & $\mathbf{1}$ & -2 \\
$(\overline{\mathbf{1 5}}, \mathbf{1})$ & $\mathbf{1}$ & 2 \\
$(\mathrm{1}, \mathbf{1})(2) \subset H)$ & $\mathbf{1}$ & 0 \\
$(\mathbf{1}, \mathbf{1})(\mathrm{U}(1)\langle 1 / 6\rangle)$ & & 0 \\
\hline
\end{tabular}

Table 17. Decomposition of $\mathbf{2 4 8}$.

\begin{tabular}{|c|c|}
\hline Representation & Multiplicity \\
\hline$(\mathbf{3 5}, \mathbf{1})$ & $-1(\mathrm{SU}(6)$ vector $)$ \\
$(\mathbf{1}, \mathbf{3})$ & $-1(\mathrm{SU}(2)$ vector $)$ \\
$(\mathbf{6}, \mathbf{2})$ & $n-\frac{2}{3} r+8$ \\
$(\mathbf{6}, \mathbf{1})$ & $n+\frac{1}{3} r+8$ \\
$(\mathbf{1}, \mathbf{2})$ & $-2+\frac{3}{2} r$ \\
$(\mathbf{2 0}, \mathbf{1})$ & $\frac{1}{2}(n-r+8)$ \\
$(\mathbf{1 5}, \mathbf{2})$ & $-2+\frac{1}{6} r$ \\
$(\mathbf{1 5}, \mathbf{1})$ & $-2+\frac{2}{3} r$ \\
$(\mathbf{1}, \mathbf{1})$ & $2 n-2 r+20$ \\
\hline
\end{tabular}

Table 18. The spectrum for the configuration No.29. 


\begin{tabular}{|c|ccc|}
\hline Rep. of SU $(5) \times \mathrm{SU}(2)_{56} \times \mathrm{SU}(2)_{78}$ & Rep. of SU(2) & Rep. of SU $(2)_{789}$ & $\mathrm{U}(1)_{\langle 1 / 4\rangle}$ charge \\
\hline$(\mathbf{1 5}, \mathbf{1}, \mathbf{1})(\mathrm{SU}(4))$ & $\mathbf{1}$ & $\mathbf{1}$ & 0 \\
$(\mathbf{4}, \mathbf{2}, \mathbf{1})$ & $\mathbf{2}$ & $\mathbf{1}$ & -1 \\
$(\overline{\mathbf{4}}, \mathbf{2}, \mathbf{1})$ & $\mathbf{2}$ & $\mathbf{1}$ & 1 \\
$(\mathbf{1}, \mathbf{3}, \mathbf{1})\left(\mathrm{SU}(2)_{56}\right)$ & $\mathbf{1}$ & $\mathbf{1}$ & 0 \\
$(\mathbf{1}, \mathbf{1}, \mathbf{1})\left(\mathrm{U}(1)_{\langle 1 / 4\rangle}\right)$ & $\mathbf{1}$ & $\mathbf{1}$ & 0 \\
$(\mathbf{4}, \mathbf{1}, \mathbf{2})$ & $\mathbf{1}$ & $\mathbf{2}$ & -1 \\
$(\overline{\mathbf{4}}, \mathbf{1}, \mathbf{2})$ & $\mathbf{1}$ & $\mathbf{2}$ & 1 \\
$(\mathbf{1}, \mathbf{2}, \mathbf{2})$ & $\mathbf{2}$ & $\mathbf{2}$ & 0 \\
$(\mathbf{1}, \mathbf{1}, \mathbf{3})\left(\mathrm{SU}(2)_{78}\right)$ & $\mathbf{1}$ & $\mathbf{1}$ & 0 \\
$(\mathbf{4}, \mathbf{1}, \mathbf{1})$ & $\mathbf{2}$ & $\mathbf{2}$ & 1 \\
$(\mathbf{1}, \mathbf{2}, \mathbf{1})$ & $\mathbf{1}$ & $\mathbf{2}$ & 2 \\
$(\mathbf{1}, \mathbf{1}, \mathbf{2})$ & $\mathbf{2}$ & $\mathbf{1}$ & 2 \\
$(\overline{\mathbf{4}}, \mathbf{1}, \mathbf{1})$ & $\mathbf{2}$ & $\mathbf{2}$ & -1 \\
$(\mathbf{1}, \mathbf{2}, \mathbf{1})$ & $\mathbf{1}$ & $\mathbf{2}$ & -2 \\
$(\mathbf{1}, \mathbf{1}, \mathbf{2})$ & $\mathbf{2}$ & $\mathbf{1}$ & -2 \\
$(\mathbf{6}, \mathbf{2}, \mathbf{1})$ & $\mathbf{1}$ & $\mathbf{2}$ & 0 \\
$(\mathbf{6}, \mathbf{1}, \mathbf{2})$ & $\mathbf{2}$ & $\mathbf{1}$ & 0 \\
$(\mathbf{4}, \mathbf{2}, \mathbf{2})$ & $\mathbf{1}$ & $\mathbf{1}$ & 1 \\
$(\overline{\mathbf{4}}, \mathbf{2}, \mathbf{2})$ & $\mathbf{1}$ & $\mathbf{1}$ & -1 \\
$(\mathbf{6}, \mathbf{1}, \mathbf{1})$ & $\mathbf{1}$ & $\mathbf{1}$ & -2 \\
$(\mathbf{6}, \mathbf{1}, \mathbf{1})$ & $\mathbf{1}$ & $\mathbf{1}$ & 2 \\
$(\mathbf{1}, \mathbf{1})\left(\mathrm{SU}(2)_{569}\right)$ & $\mathbf{3}$ & $\mathbf{1}$ & 0 \\
$(\mathbf{1}, \mathbf{1}, \mathbf{1})\left(\mathrm{SU}(2)_{789}\right)$ & $\mathbf{1}$ & $\mathbf{3}$ & 0 \\
$\mathbf{6}$ & & & \\
\hline
\end{tabular}

Table 19. Decomposition of 248.

whereas the $\mathrm{U}(1)$ in $H\left(\equiv \mathrm{U}(1)_{\langle 1 / 4\rangle}\right)$ is associated with the $E_{8}$ root

$$
-\frac{1}{3}\left(\mathbf{e}_{1}+\mathbf{e}_{2}+\mathbf{e}_{3}+\mathbf{e}_{4}\right)+\frac{2}{3}\left(\mathbf{e}_{5}+\mathbf{e}_{6}+\mathbf{e}_{7}+\mathbf{e}_{8}\right)-\frac{4}{3} \mathbf{e}_{9} .
$$

The decomposition of $E_{8}$ is in table 19. In this case we assume instantons $r, \tilde{r}$ and $12+n-r-\tilde{r}$ in $\mathrm{SU}(2)_{56}, \mathrm{SU}(2)_{78}$ and $\mathrm{U}(1)_{\langle 1 / 4\rangle}$ so that the $\mathrm{SU}(2)$ 's in $H$ are treated in a symmetric way. The spectrum of No.29 is as shown in table 20. (Again, representations with ${ }^{-}$and without ${ }^{-}$are identified.)

\section{Charged matter spectrum from F-theory geometry}

In this appendix, we present the explicit form of the series expansions of $f_{\text {res }}, g_{\text {res }}$ and $\Delta_{\text {res }}$ and the resulting charged matter spectrum. 


\begin{tabular}{|c|c|}
\hline Representation & Multiplicity \\
\hline$(\mathbf{1 5}, \mathbf{1}, \mathbf{1})$ & $-1(\mathrm{SU}(4)$ vector $)$ \\
$(\mathbf{1}, \mathbf{3}, \mathbf{1})$ & $-1\left(\mathrm{SU}(2)_{56}\right.$ vector $)$ \\
$(\mathbf{1}, \mathbf{3}, \mathbf{1})$ & $-1\left(\mathrm{SU}(2)_{78}\right.$ vector $)$ \\
$(\mathbf{4}, \mathbf{2}, \mathbf{1})$ & $\frac{1}{2}(n+r-\tilde{r})+2$ \\
$(\mathbf{4}, \mathbf{1}, \mathbf{2})$ & $\frac{1}{2}(n-r+\tilde{r})+2$ \\
$(\mathbf{1}, \mathbf{2}, \mathbf{2})$ & $r+\tilde{r}-4$ \\
$(\mathbf{4}, \mathbf{1}, \mathbf{1})$ & $n+r+\tilde{r}+4$ \\
$(\mathbf{1}, \mathbf{2}, \mathbf{1})$ & $2 n-2 r-\tilde{r}+20$ \\
$(\mathbf{1}, \mathbf{1}, \mathbf{2})$ & $2 n-r-2 \tilde{r}+20$ \\
$(\mathbf{6}, \mathbf{2}, \mathbf{1})$ & $\frac{\tilde{r}}{2}-2$ \\
$(\mathbf{6}, \mathbf{1}, \mathbf{2})$ & $\frac{r}{2}-2$ \\
$(\mathbf{4}, \mathbf{2}, \mathbf{2})$ & $\frac{1}{4}(n-r-\tilde{r})+1$ \\
$(\mathbf{6}, \mathbf{1}, \mathbf{1})$ & $n-r-\tilde{r}+10$ \\
$(\mathbf{1}, \mathbf{1}, \mathbf{1})$ & $2 r+2 \tilde{r}-7$ \\
\hline
\end{tabular}

Table 20. The spectrum for the configuration No.22.

\section{C.1 $\quad G=\mathrm{SU}(2) \times \mathrm{SU}(2) \times \mathrm{SU}(2)(r \neq 0)$}

The construction of the geometry is given in section 5.3. The series expansions of $f_{\text {res }}, g_{\text {res }}$ and $\Delta_{\text {res }}$ near each of the three lines $z=0, \tilde{z}=0$ and $\hat{z}=0$ are obtained as follows:

$$
\begin{aligned}
f_{\text {res }}= & -\frac{1}{3} t_{r}^{2} K_{4+2 n-r}^{2}+(\cdots) t_{r} z+o\left(z^{2}\right) \\
= & -\frac{1}{3} t_{r}^{2} \tilde{K}_{4+2 n-r}^{2}+(\cdots) t_{r} \tilde{z}+o\left(\tilde{z}^{2}\right) \\
= & -\frac{1}{3} t_{r}^{2} \hat{K}_{4+2 n-r}^{2}+(\cdots) t_{r} \hat{z}+o\left(\hat{z}^{2}\right) \\
g_{\text {res }}= & \frac{2}{27} t_{r}^{3} K_{4+2 n-r}^{3}+(\cdots) t_{r}^{2} K_{4+2 n-r} z+(\cdots) t_{r} z^{2}+o\left(z^{3}\right) \\
= & \frac{2}{27} t_{r}^{3} \tilde{K}_{4+2 n-r}^{3}+(\cdots) t_{r}^{2} \tilde{K}_{4+2 n-r} \tilde{z}+(\cdots) t_{r} \tilde{z}^{2}+o\left(\tilde{z}^{3}\right) \\
= & \frac{2}{27} t_{r}^{3} \hat{K}_{4+2 n-r}^{3}+(\cdots) t_{r}^{2} \hat{K}_{4+2 n-r} \hat{z}+(\cdots) t_{r} \hat{z}^{2}+o\left(\hat{z}^{3}\right) \\
\Delta_{\mathrm{res}}= & t_{r}^{4} h_{n-r}^{2} h_{n-r}^{2} K_{4+2 n-r}^{2} Q_{16+2 n+2 r} z^{2}+(\cdots) t_{r}^{3} h_{n-r} h_{n-r}^{\prime} z^{3} \\
& +(\cdots) t_{r}^{2} z^{4}+(\cdots) t_{r} z^{5}+o\left(z^{6}\right) \\
= & t_{r}^{4} h_{n-r}^{2}\left(h_{n-r}-h_{n-r}^{\prime}\right)^{2} \tilde{K}_{4+2 n-r}^{2} \tilde{Q}_{16+2 n+2 r} \tilde{z}^{2}+(\cdots) t_{r}^{3} h_{n-r}\left(h_{n-r}-h_{n-r}^{\prime}\right) \tilde{z}^{3} \\
& +(\cdots) t_{r}^{2} \tilde{z}^{4}+(\cdots) t_{r} \tilde{z}^{5}+o\left(\tilde{z}^{6}\right) \\
= & t_{r}^{4} h_{n-r}^{\prime 2}\left(h_{n-r}-h_{n-r}^{\prime}\right)^{2} \hat{K}_{4+2 n-r}^{2} \hat{Q}_{16+2 n+2 r} \hat{z}^{2}+(\cdots) t_{r}^{3} h_{n-r}^{\prime}\left(h_{n-r}-h_{n-r}^{\prime}\right) \hat{z}^{3} \\
& +(\cdots) t_{r}^{2} \hat{z}^{4}+(\cdots) t_{r} \hat{z}^{5}+o\left(\hat{z}^{6}\right),
\end{aligned}
$$


where

$$
\begin{aligned}
& K_{4+2 n-r}=s_{4+2 n-2 r} t_{r}+\frac{1}{3} q_{4+r} \hat{\sigma}_{2}, \\
& \tilde{K}_{4+2 n-r}=s_{4+2 n-2 r} t_{r}-k_{4+n-r} t_{r} h_{n-r}+\frac{1}{3} q_{4+r} h_{n-r}\left(h_{n-r}-h_{n-r}^{\prime}\right), \\
& \hat{K}_{4+2 n-r}=s_{4+2 n-2 r} t_{r}-k_{4+n-r} t_{r} h_{n-r}^{\prime}+\frac{1}{3} q_{4+r} h_{n-r}^{\prime}\left(h_{n-r}^{\prime}-h_{n-r}\right) .
\end{aligned}
$$

$Q_{16+2 n+2 r}, \tilde{Q}_{16+2 n+2 r}$ and $\hat{Q}_{16+2 n+2 r}$ are degree $16+2 n+2 r$ irreducible polynomials. The resulting charged matter spectrum is given as follows:

\begin{tabular}{|l|c|ccc|c|c|}
\hline \multicolumn{1}{|c|}{ Zero } & Degree & $\operatorname{ord}\left(f_{\text {res }}\right)$ ord $\left(g_{\text {res }}\right)$ ord $\left(\Delta_{\text {res }}\right)$ & Enhancement & Matter \\
\hline$K, \tilde{K}, \hat{K}$ & $4+2 n-r$ & 1 & 2 & 3 & $A_{1} \rightarrow A_{1}\left(I_{2} \rightarrow I I I\right)$ & none \\
\hline$Q_{16+2 n+2 r}$ & $16+2 n+2 r$ & 0 & 0 & 3 & $A_{1} \rightarrow A_{2}$ & $(\mathbf{2}, \mathbf{1}, \mathbf{1})$ \\
$\tilde{Q}_{16+2 n+2 r}$ & $16+2 n+2 r$ & 0 & 0 & 3 & $A_{1} \rightarrow A_{2}$ & $(\mathbf{1}, \mathbf{2}, \mathbf{1})$ \\
$\hat{Q}_{16+2 n+2 r}$ & $16+2 n+2 r$ & 0 & 0 & 3 & $A_{1} \rightarrow A_{2}$ & $(\mathbf{1}, \mathbf{1}, \mathbf{2})$ \\
\hline$h_{n-r}$ & $n-r$ & 0 & 0 & 4 & $A_{1} \oplus A_{1} \rightarrow A_{3}$ & $(\mathbf{2}, \mathbf{2}, \mathbf{1})$ \\
$h_{n-r}^{\prime}$ & $n-r$ & 0 & 0 & 4 & $A_{1} \oplus A_{1} \rightarrow A_{3}$ & $(\mathbf{2}, \mathbf{1}, \mathbf{2})$ \\
$h_{n-r}-h_{n-r}^{\prime}$ & $n-r$ & 0 & 0 & 4 & $A_{1} \oplus A_{1} \rightarrow A_{3}$ & $(\mathbf{1}, \mathbf{2}, \mathbf{2})$ \\
\hline$t_{r}$ & $r$ & 2 & 3 & 6 & $A_{1} \oplus A_{1} \oplus A_{1} \rightarrow D_{4}$ & $\frac{1}{2}(\mathbf{2}, \mathbf{2}, \mathbf{2})$ \\
\hline
\end{tabular}

\section{C.2 $G=\mathrm{SU}(3) \times \mathrm{SU}(2) \times \mathrm{SU}(2)(\mathrm{No.12})$}

The construction of the geometry is given in section 6.1. The series expansions of $f_{\text {res }}, g_{\text {res }}$ and $\Delta_{\text {res }}$ near each singularity are obtained as follows:

$$
\begin{aligned}
f_{\text {res }} & =-\frac{1}{3} p_{2+n}^{4}+(\cdots) p_{2+n} z+o\left(z^{2}\right) \\
& =-\frac{1}{3} \tilde{K}_{4+2 n}^{2}+o(\tilde{z}) \\
& =-\frac{1}{3} \hat{K}_{4+2 n}^{2}+o(\hat{z}), \\
g_{\text {res }} & =\frac{2}{27} p_{2+n}^{6}+(\cdots) p_{2+n}^{3} z+o\left(z^{2}\right) \\
& =\frac{2}{27} \tilde{K}_{4+2 n}^{3}+(\cdots) \tilde{K}_{4+2 n} \tilde{z}+o\left(\tilde{z}^{2}\right) \\
& =\frac{2}{27} \hat{K}_{4+2 n}^{3}+(\cdots) \hat{K}_{4+2 n} \hat{z}+o\left(\hat{z}^{2}\right), \\
\Delta_{\text {res }} & =h_{n}^{2} h_{n}^{\prime 2} p_{2+n}^{3} q_{6} R_{12+2 n} z^{3}+(\cdots) h_{n} h_{n}^{\prime} z^{4}+o\left(z^{5}\right) \\
& =\tilde{K}_{4+2 n}^{2} h_{n}^{3}\left(h_{n}-h_{n}^{\prime}\right)^{2} \tilde{S}_{16+n} \tilde{z}^{2}+(\cdots) h_{n}^{2}\left(h_{n}-h_{n}^{\prime}\right) \tilde{z}^{3}+(\cdots) h_{n} \tilde{z}^{4}+o\left(\tilde{z}^{5}\right) \\
& =\hat{K}_{4+2 n}^{2} h_{n}^{\prime 3}\left(h_{n}-h_{n}^{\prime}\right)^{2} \hat{S}_{16+n} \hat{z}^{2}+(\cdots) h_{n}^{\prime 2}\left(h_{n}-h_{n}^{\prime}\right) \hat{z}^{3}+(\cdots) h_{n}^{\prime} \hat{z}^{4}+o\left(\hat{z}^{5}\right) .
\end{aligned}
$$

Here

$$
\begin{aligned}
& \tilde{K}_{4+2 n}=p_{2+n}^{2}-A_{21} h_{n}+A_{22} h_{n}^{2}, \\
& \hat{K}_{4+2 n}=p_{2+n}^{2}-A_{21} h_{n}^{\prime}+A_{22} h_{n}^{\prime 2} .
\end{aligned}
$$


$R_{12+2 n}, \tilde{S}_{16+n}$ and $\hat{S}_{16+n}$ are irreducible polynomials of orders $12+2 n, 16+n$ and $16+n$, respectively. The charged matter spectrum is then read

\begin{tabular}{|l|c|ccc|c|c|}
\hline \multicolumn{1}{|c|}{ Zero } & Degree & ord $\left(f_{\text {res }}\right)$ & ord $\left(g_{\text {res }}\right)$ & ord $\left(\Delta_{\text {res }}\right)$ & Enhancement & Matter \\
\hline$p_{2+n}$ & $2+n$ & 2 & 2 & 4 & $A_{2} \rightarrow A_{2}\left(I_{3} \rightarrow I V\right)$ & none \\
$q_{6}$ & 6 & 0 & 0 & 4 & $A_{2} \rightarrow A_{3}$ & $(\mathbf{3}, \mathbf{1}, \mathbf{1})$ \\
$R_{12+2 n}$ & $12+2 n$ & 0 & 0 & 4 & $A_{2} \rightarrow A_{3}$ & $(\mathbf{3}, \mathbf{1}, \mathbf{1})$ \\
\hline$\tilde{K}_{4+2 n}$ & $4+2 n$ & 1 & 2 & 3 & $A_{1} \rightarrow A_{1}\left(I_{2} \rightarrow I I I\right)$ & none \\
$\tilde{S}_{16+n}$ & $16+n$ & 0 & 0 & 3 & $A_{1} \rightarrow A_{2}$ & $(\mathbf{1}, \mathbf{2}, \mathbf{1})$ \\
\hline$\hat{K}_{4+2 n}$ & $4+2 n$ & 1 & 2 & 3 & $A_{1} \rightarrow A_{1}\left(I_{2} \rightarrow I I I\right)$ & none \\
$\hat{S}_{16+n}$ & $16+n$ & 0 & 0 & 3 & $A_{1} \rightarrow A_{2}$ & $(\mathbf{1}, \mathbf{1}, \mathbf{2})$ \\
\hline$h_{n}$ & $n$ & 0 & 0 & 5 & $A_{2} \oplus A_{1} \rightarrow A_{4}$ & $(\mathbf{3 , 2}, \mathbf{1})$ \\
$h_{n}^{\prime}$ & $n$ & 0 & 0 & 5 & $A_{2} \oplus A_{1} \rightarrow A_{4}$ & $(\mathbf{3}, \mathbf{1}, \mathbf{2})$ \\
$h_{n}-h_{n}^{\prime}$ & $n$ & 0 & 0 & 4 & $A_{1} \oplus A_{1} \rightarrow A_{3}$ & $(\mathbf{1}, \mathbf{2}, \mathbf{2})$ \\
\hline
\end{tabular}

\section{C.3 $G=\mathrm{SU}(5) \times \mathrm{SU}(2)(\mathrm{No.17})$}

The construction of the geometry is given in section 6.2. The series expansions near the two lines $z=0$ and $\tilde{z}=z+h_{n}=0$ are given by

$$
\begin{aligned}
f_{\text {res }} & =-\frac{1}{3} p_{2+n}^{4}+(\cdots) p_{2+n}^{2} z+o\left(z^{2}\right) \\
& =-\frac{1}{3} \tilde{K}_{4+2 n}^{2}+o(\tilde{z}) \\
g_{\text {res }} & =\frac{2}{27} p_{2+n}^{6}+(\cdots) p_{2+n}^{4} z+(\cdots) p_{2+n}^{2} z^{2}+o\left(z^{3}\right) \\
& =\frac{2}{27} \tilde{K}_{4+2 n}^{3}+(\cdots) \tilde{K}_{4+2 n} \tilde{z}+o\left(\tilde{z}^{2}\right), \\
\Delta_{\text {res }} & =h_{n}^{2} p_{2+n}^{4} q_{6} R_{10+n} z^{5}+(\cdots) h_{n} p_{n+2}^{2} z^{6}+o\left(z^{7}\right) \\
& =\tilde{K}_{4+2 n}^{2} h_{n}^{5} \tilde{S}_{16+n} \tilde{z}^{2}+(\cdots) h_{n}^{4} \tilde{z}^{3}+\cdots+(\cdots) h_{n} \tilde{z}^{6}+o\left(\tilde{z}^{7}\right) .
\end{aligned}
$$

Here $R_{10+n}$ is a degree $10+n$ irreducible polynomial. $\tilde{S}_{16+n}$ is the same one contained in (C.8) and $\tilde{K}_{4+2 n}$ is given in (C.9). Charged matter spectrum is obtained as follows:

\begin{tabular}{|l|c|ccc|c|c|}
\hline Zero & Degree & ord $\left(f_{\text {res }}\right)$ & ord $\left(g_{\text {res }}\right)$ & ord $\left(\Delta_{\text {res }}\right)$ & Enhancement & Matter \\
\hline$p_{2+n}$ & $2+n$ & 2 & 3 & 7 & $A_{4} \rightarrow D_{5}$ & $(\mathbf{1 0}, \mathbf{1})$ \\
$q_{6}$ & 6 & 0 & 0 & 6 & $A_{4} \rightarrow A_{5}$ & $(\mathbf{5}, \mathbf{1})$ \\
$R_{10+n}$ & $10+n$ & 0 & 0 & 6 & $A_{4} \rightarrow A_{5}$ & $(\mathbf{5}, \mathbf{1})$ \\
\hline$\tilde{K}_{4+2 n}$ & $4+2 n$ & 1 & 2 & 3 & $A_{1} \rightarrow A_{1}\left(I_{2} \rightarrow I I I\right)$ & none \\
$\tilde{S}_{16+n}$ & $16+n$ & 0 & 0 & 3 & $A_{1} \rightarrow A_{2}$ & $(\mathbf{1}, \mathbf{2})$ \\
\hline$h_{n}$ & $n$ & 0 & 0 & 7 & $A_{4} \oplus A_{1} \rightarrow A_{6}$ & $(\mathbf{5}, \mathbf{2})$ \\
\hline
\end{tabular}




\section{C.4 $G=\mathrm{SU}(3) \times \mathrm{SU}(4)(\mathrm{No.19})$}

The construction of the geometry is given in section 6.3. The expansions near the two lines $z=0$ and $\tilde{z}=z+h_{n}=0$ are given by

$$
\begin{aligned}
f_{\text {res }} & =-\frac{1}{3} p_{2+n}^{4}+(\cdots) p_{2+n} z+o\left(z^{2}\right) \\
& =-\frac{1}{3} \tilde{p}_{2+n}^{4}+(\cdots) \tilde{p}_{2+n}^{2} \tilde{z}+o\left(\tilde{z}^{2}\right) \\
g_{\text {res }} & =\frac{2}{27} p_{2+n}^{6}+(\cdots) p_{2+n}^{3} z+o\left(z^{2}\right) \\
& =\frac{2}{27} \tilde{p}_{2+n}^{6}+(\cdots) \tilde{p}_{2+n}^{4} \tilde{z}+(\cdots) \tilde{p}_{2+n}^{2} \tilde{z}^{2}+o\left(\tilde{z}^{3}\right), \\
\Delta_{\text {res }} & =h_{n}^{4} p_{2+n}^{3} q_{6} R_{12+2 n} z^{3}+(\cdots) h_{n}^{3} z^{4}+(\cdots) h_{n}^{2} z^{5}+(\cdots) h_{n} z^{6}+o\left(z^{7}\right) \\
& =h_{n}^{3} \tilde{p}_{2+n}^{4} \tilde{S}_{8+n} \tilde{S}_{8}^{\prime} \tilde{z}^{4}+(\cdots) h_{n}^{2} \tilde{p}_{2+n}^{2} \tilde{z}^{5}+(\cdots) h_{n} \tilde{z}^{6}+o\left(\tilde{z}^{7}\right) .
\end{aligned}
$$

\begin{tabular}{|c|c|c|c|c|c|c|}
\hline Zero & Degree & $\operatorname{ord}\left(f_{\text {res }}\right)$ & $\operatorname{ord}\left(g_{\mathrm{res}}\right)$ & $\operatorname{ord}\left(\Delta_{\text {res }}\right)$ & Enhancement & Matter \\
\hline$p_{2+n}$ & $2+n$ & 2 & 2 & 4 & $A_{2} \rightarrow A_{2}\left(I_{3} \rightarrow I V\right)$ & none \\
\hline$q_{6}$ & 6 & 0 & 0 & 4 & $A_{2} \rightarrow A_{3}$ & $(\mathbf{3}, \mathbf{1})$ \\
\hline$R_{12+2 n}$ & $12+2 n$ & 0 & 0 & 4 & $A_{2} \rightarrow A_{3}$ & $(\mathbf{3}, \mathbf{1})$ \\
\hline$\tilde{p}_{2+n}$ & $2+n$ & 2 & 3 & 6 & $A_{3} \rightarrow D_{4}$ & $(1,6)$ \\
\hline$\tilde{S}_{8+n}$ & $8+n$ & 0 & 0 & 5 & $A_{3} \rightarrow A_{4}$ & $(1,4)$ \\
\hline$\tilde{S}_{8}^{\prime}$ & 8 & 0 & 0 & 5 & $A_{3} \rightarrow A_{4}$ & $(\mathbf{1}, \mathbf{4})$ \\
\hline$h_{n}$ & $n$ & 0 & 0 & 7 & $A_{2} \oplus A_{3} \rightarrow A_{6}$ & $(3,4)$ \\
\hline
\end{tabular}

Charged matter spectrum is obtained as follows:

\section{C.5 $\quad G=\mathrm{SU}(7)(\mathrm{No.25})$}

The construction of the geometry is given in section 6.4. The series expansions are given by

$$
\begin{aligned}
f_{\mathrm{res}} & =-\frac{1}{3} p_{2+n}^{4}-\frac{2}{3} p_{2+n}^{2} r_{4+n} z+o\left(z^{2}\right) \\
g_{\mathrm{res}} & =\frac{2}{27} p_{2+n}^{6}+\frac{2}{9} p_{2+n}^{4} r_{4+n} z+(\cdots) p_{2+n}^{2} z^{2}+o\left(z^{3}\right), \\
\Delta_{\mathrm{res}} & =-\frac{4}{3} p_{2+n}^{4} q_{6} R_{10+n} z^{7}+(\cdots) p_{2+n}^{2} z^{8}+o\left(z^{9}\right),
\end{aligned}
$$

with

$$
R_{10+n} \equiv A_{22}^{2} p_{2+n}+3 \tilde{A}_{44} p_{2+n}-3 q_{6} r_{4+n} .
$$

Charged matter spectrum is obtained as follows:

\begin{tabular}{|l|c|ccc|c|c|}
\hline Zero & Degree & ord $\left(f_{\text {res }}\right)$ & ord $\left(g_{\text {res }}\right)$ & ord $\left(\Delta_{\text {res }}\right)$ & Enhancement & Matter \\
\hline$p_{2+n}$ & $2+n$ & 2 & 3 & 9 & $A_{6} \rightarrow D_{7}$ & $\mathbf{2 1}$ \\
$q_{6}$ & 6 & 0 & 0 & 8 & $A_{6} \rightarrow A_{7}$ & $\mathbf{7}$ \\
$R_{10+n}$ & $10+n$ & 0 & 0 & 8 & $A_{6} \rightarrow A_{7}$ & $\mathbf{7}$ \\
\hline
\end{tabular}




\section{C.6 $G=\mathrm{SU}(4) \times \mathrm{SU}(2) \times \mathrm{SU}(2)(\mathrm{No.22})$}

The construction of the geometry is given in section 7.1. The expansions near each line of the singularity are given by

$$
\begin{aligned}
f_{\text {res }}= & -\frac{1}{3} r_{n-4}^{4} q_{6}^{4}+(\cdots) r_{n-4}^{2} q_{6}^{2} z+o\left(z^{2}\right) \\
= & -\frac{1}{3} r_{n-4}^{2} \tilde{L}_{8+n}^{2}+(\cdots) r_{n-4} \tilde{z}+o\left(\tilde{z}^{2}\right) \\
= & -\frac{1}{3} \hat{K}_{4+2 n}^{2}+o(\hat{z}), \\
g_{\text {res }}= & \frac{2}{27} r_{n-4}^{6} q_{6}^{6}+(\cdots) r_{n-4}^{4} q_{6}^{4} z+(\cdots) r_{n-4}^{2} q_{6}^{2} z^{2}+o\left(z^{3}\right) \\
= & \frac{2}{27} r_{n-4}^{3} \tilde{L}_{8+n}^{3}+(\cdots) r_{n-4}^{2} \tilde{L}_{8+n} \tilde{z}+(\cdots) r_{n-4} \tilde{z}^{2}+o\left(\tilde{z}^{3}\right) \\
= & \frac{2}{27} \hat{K}_{4+2 n}^{3}+(\cdots) \hat{K}_{4+2 n} \hat{z}+o\left(\hat{z}^{2}\right), \\
\Delta_{\text {res }}= & s_{4}^{2} h_{n}^{\prime 2} r_{n-4}^{4} q_{6}^{4} T_{8+2 n} z^{4}+(\cdots) s_{4} h_{n}^{\prime} r_{n-4}^{2} q_{6}^{2} z^{5}+(\cdots) r_{n-4} z^{6}+o\left(z^{7}\right) \\
= & \tilde{L}_{8+n}^{2} s_{4}^{4} r_{n-4}^{5}\left(r_{n-4} s_{4}-h_{n}^{\prime}\right)^{2} \tilde{U}_{12+n} \tilde{z}^{2}+(\cdots) s_{4}^{3} r_{n-4}^{4}\left(r_{n-4} s_{4}-h_{n}^{\prime}\right) \tilde{z}^{3} \\
& +(\cdots) s_{4}^{2} r_{n-4}^{3} \tilde{z}^{4}+(\cdots) s_{4} r_{n-4}^{2} \tilde{z}^{5}+(\cdots) r_{n-4} \tilde{z}^{6}+o\left(\tilde{z}^{7}\right) \\
= & \hat{K}_{4+2 n}^{2} h_{n}^{\prime 4}\left(r_{n-4} s_{4}-h_{n}^{\prime}\right)^{2} \hat{V}_{16} \hat{z}^{2}+(\cdots) h_{n}^{\prime 3}\left(r_{n-4} s_{4}-h_{n}^{\prime}\right) \hat{z}^{3}+(\cdots) h_{n}^{\prime 2} \hat{z}^{4} \\
& +(\cdots) h_{n}^{\prime} \hat{z}^{5}+o\left(\hat{z}^{6}\right), \\
& (\cdots
\end{aligned}
$$

where

$$
\begin{aligned}
\tilde{L}_{8+n} & =r_{n-4} q_{6}^{2}-\left(\frac{1}{3} A_{22}^{2}+\tilde{A}_{44}\right) r_{n-4} s_{4}-h_{n}^{\prime} s_{4}^{2}+A_{22} r_{n-4} s_{4}^{2}, \\
\hat{K}_{4+2 n} & =r_{n-4}^{2} q_{6}^{2}-\left(\frac{1}{3} A_{22}^{2}+\tilde{A}_{44}\right) r_{n-4} h_{n}^{\prime}-s_{4} h_{n}^{\prime 2}+A_{22} h_{n}^{\prime 2} .
\end{aligned}
$$

$T_{8+n}, \tilde{U}_{12+n}$ and $\hat{V}_{16}$ are irreducible polynomials with degrees $8+n, 12+n$ and 16 .

Charged matter spectrum can be read from these expansions. The result is

\begin{tabular}{|l|c|ccc|c|c|}
\hline \multicolumn{1}{|c|}{ Zero } & Degree & $\operatorname{ord}\left(f_{\text {res }}\right)$ & ord $\left(g_{\text {res }}\right)$ & ord $\left(\Delta_{\text {res }}\right)$ & Enhancement & Matter \\
\hline$q_{6}$ & 6 & 2 & 3 & 6 & $A_{3} \rightarrow D_{4}$ & $(\mathbf{6 , 1}, \mathbf{1})$ \\
$T_{8+2 n}$ & $8+2 n$ & 0 & 0 & 5 & $A_{3} \rightarrow A_{4}$ & $(\mathbf{4}, \mathbf{1}, \mathbf{1})$ \\
\hline$\tilde{L}_{8+n}$ & $8+n$ & 1 & 2 & 3 & $A_{1} \rightarrow A_{1}\left(I_{2} \rightarrow I I I\right)$ & none \\
$\tilde{U}_{12+n}$ & $12+n$ & 0 & 0 & 3 & $A_{1} \rightarrow A_{2}$ & $(\mathbf{1}, \mathbf{2}, \mathbf{1})$ \\
\hline$\hat{K}_{4+2 n}$ & $4+2 n$ & 1 & 2 & 3 & $A_{1} \rightarrow A_{1}\left(I_{2} \rightarrow I I I\right)$ & none \\
$\hat{V}_{16}$ & 16 & 0 & 0 & 3 & $A_{1} \rightarrow A_{2}$ & $(\mathbf{1}, \mathbf{1}, \mathbf{2})$ \\
\hline$s_{4}$ & 4 & 0 & 0 & 6 & $A_{3} \oplus A_{1} \rightarrow A_{5}$ & $(\mathbf{4}, \mathbf{2}, \mathbf{1})$ \\
$r_{n-4}$ & $n-4$ & 2 & 3 & 7 & $A_{3} \oplus A_{1} \rightarrow D_{5}$ & $\frac{1}{2}(\mathbf{6 , 2} \mathbf{1})$ \\
\hline$h_{n}^{\prime}$ & $n$ & 0 & 0 & 6 & $A_{3} \oplus A_{1} \rightarrow A_{5}$ & $(\mathbf{4}, \mathbf{1}, \mathbf{2})$ \\
\hline$r_{n-4} s_{4}-h_{n}^{\prime}$ & $n$ & 0 & 0 & 4 & $A_{1} \oplus A_{1} \rightarrow A_{3}$ & $(\mathbf{1}, \mathbf{2}, \mathbf{2})$ \\
\hline
\end{tabular}


In the third line from the bottom, enhancement $\mathrm{SU}(4) \times \mathrm{SU}(2) \rightarrow \mathrm{SO}(10)$ occurs and a half-hypermultiplet $\frac{1}{2}(\mathbf{6}, \mathbf{2}, \mathbf{1})$ appears. (The third $\mathrm{SU}(2)$ is not concerned with this enhancement.) The reason is as follows. The maximal embedding corresponding to this enhancement is $\mathrm{SO}(10) \supset \mathrm{SU}(4) \times \mathrm{SU}(2) \times \mathrm{SU}(2)$, whose branching is given by

$$
45=(15,1,1) \oplus(1,3,1) \oplus(1,1,3) \oplus(6,2,2) .
$$

As explained below eq. (5.32), the representation coupled to $\mathbf{2}$ is pseudo-real, yielding a half-hypermultiplet. In this case, it is $\frac{1}{2}(\mathbf{6}, \mathbf{2})$.

\section{C.7 $G=\mathrm{SU}(6) \times \mathrm{SU}(2)(\mathrm{No.29})$}

The construction of the geometry is given in section 7.2. The expansions are given by

$$
\begin{aligned}
f_{\text {res }} & =-\frac{1}{3} r_{n-4}^{4} q_{6}^{4}+(\cdots) r_{n-4}^{3} q_{6}^{2} z+(\cdots) r_{n-4} z^{2}+o\left(z^{3}\right) \\
& =-\frac{1}{3} \hat{K}_{4+2 n}^{2}+o(\hat{z}) \\
g_{\text {res }} & =\frac{2}{27} r_{n-4}^{6} q_{6}^{6}+(\cdots) r_{n-4}^{5} q_{6}^{4} z+(\cdots) r_{n-4}^{3} q_{6}^{2} z^{2}+(\cdots) r_{n-4}^{2} z^{3}+o\left(z^{4}\right) \\
& =\frac{2}{27} \hat{K}_{4+2 n}^{3}+(\cdots) \tilde{K}_{4+2 n} \hat{z}+o\left(\hat{z}^{2}\right), \\
\Delta_{\text {res }} & =r_{n-4}^{3} q_{6}^{4} h_{n}^{\prime 2} R_{12+n} z^{6}+(\cdots) r_{n-4}^{2} q_{6}^{2} h_{n}^{\prime} z^{7}+o\left(z^{8}\right) \\
& =\hat{K}_{4+2 n}^{2} h_{n}^{\prime 6} \hat{S}_{16} \hat{z}^{2}+(\cdots) h_{n}^{\prime 5} \hat{z}^{3}+\cdots+(\cdots) h_{n}^{\prime} \hat{z}^{7}+o\left(\hat{z}^{8}\right),
\end{aligned}
$$

where $R_{12+n}$ and $\hat{S}_{16}$ are degree $12+n$ and 16 irreducible polynomials. $\hat{K}_{4+2 n}$ is the one given in (C.25) with $s_{4}$ being set to zero.

Charged matter spectrum is given as follows:

\begin{tabular}{|l|c|ccc|c|c|}
\hline Zero & Degree & ord $\left(f_{\text {res }}\right)$ & ord $\left(g_{\text {res }}\right)$ & ord $\left(\Delta_{\text {res }}\right)$ & Enhancement & Matter \\
\hline$r_{n-4}$ & $n-4$ & 3 & 4 & 8 & $A_{5} \rightarrow E_{6}$ & $\frac{1}{2}(\mathbf{2 0}, \mathbf{1})$ \\
$q_{6}$ & 6 & 2 & 3 & 8 & $A_{5} \rightarrow D_{6}$ & $(\mathbf{1 5}, \mathbf{1})$ \\
$R_{12+n}$ & $12+n$ & 0 & 0 & 7 & $A_{5} \rightarrow A_{6}$ & $(\mathbf{6}, \mathbf{1})$ \\
\hline$\hat{K}_{4+2 n}$ & $4+2 n$ & 1 & 2 & 3 & $A_{1} \rightarrow A_{1}\left(I_{2} \rightarrow I I I\right)$ & none \\
$\hat{S}_{16}$ & 16 & 0 & 0 & 3 & $A_{1} \rightarrow A_{2}$ & $(\mathbf{1}, \mathbf{2})$ \\
\hline$h_{n}^{\prime}$ & $n$ & 0 & 0 & 8 & $A_{5} \oplus A_{1} \rightarrow A_{7}$ & $(\mathbf{6}, \mathbf{2})$ \\
\hline
\end{tabular}

In the first line, at $r_{n-4}=0$, enhancement $\mathrm{SU}(6) \rightarrow E_{6}$ occurs. The corresponding maximal embedding is $E_{6} \supset \mathrm{SU}(6) \times \mathrm{SU}(2)$ and the branching is given by

$$
78=(35,1) \oplus(3,1) \oplus(20,2) .
$$

Therefore a half-hypermultiplet $\frac{1}{2} \mathbf{2 0}$ appears at this point.

Open Access. This article is distributed under the terms of the Creative Commons Attribution License (CC-BY 4.0), which permits any use, distribution and reproduction in any medium, provided the original author(s) and source are credited. 


\section{References}

[1] C. Vafa, Evidence for F-theory, Nucl. Phys. B 469 (1996) 403 [hep-th/9602022] [INSPIRE].

[2] D.R. Morrison and C. Vafa, Compactifications of F-theory on Calabi-Yau threefolds. 1, Nucl. Phys. B 473 (1996) 74 [hep-th/9602114] [INSPIRE].

[3] D.R. Morrison and C. Vafa, Compactifications of F-theory on Calabi-Yau threefolds. 2., Nucl. Phys. B 476 (1996) 437 [hep-th/9603161] [INSPIRE].

[4] E. Witten, Strong coupling expansion of Calabi-Yau compactification, Nucl. Phys. B 471 (1996) 135 [hep-th/9602070] [INSPIRE].

[5] R. Blumenhagen, M. Cvetič and T. Weigand, Spacetime instanton corrections in $4 D$ string vacua: The seesaw mechanism for D-brane models, Nucl. Phys. B 771 (2007) 113 [hep-th/0609191] [INSPIRE].

[6] R. Donagi and M. Wijnholt, Model Building with F-theory, Adv. Theor. Math. Phys. 15 (2011) 1237 [arXiv:0802.2969] [INSPIRE].

[7] C. Beasley, J.J. Heckman and C. Vafa, GUTs and Exceptional Branes in F-theory - I, JHEP 01 (2009) 058 [arXiv:0802.3391] [INSPIRE].

[8] C. Beasley, J.J. Heckman and C. Vafa, GUTs and Exceptional Branes in F-theory - II: Experimental Predictions, JHEP 01 (2009) 059 [arXiv:0806.0102] [INSPIRE].

[9] R. Donagi and M. Wijnholt, Breaking GUT Groups in F-theory, Adv. Theor. Math. Phys. 15 (2011) 1523 [arXiv:0808.2223] [INSPIRE].

[10] H. Hayashi, T. Kawano, R. Tatar and T. Watari, Codimension-3 Singularities and Yukawa Couplings in F-theory, Nucl. Phys. B 823 (2009) 47 [arXiv:0901.4941] [InSPIRE].

[11] R. Donagi and M. Wijnholt, Higgs Bundles and UV Completion in F-theory, Commun. Math. Phys. 326 (2014) 287 [arXiv:0904.1218] [INSPIRE].

[12] J.J. Heckman, J. Marsano, N. Saulina, S. Schäfer-Nameki and C. Vafa, Instantons and SUSY breaking in F-theory, arXiv:0808.1286 [INSPIRE].

[13] J. Marsano, N. Saulina and S. Schäfer-Nameki, Gauge Mediation in F-theory GUT Models, Phys. Rev. D 80 (2009) 046006 [arXiv:0808.1571] [INSPIRE].

[14] J.J. Heckman and C. Vafa, F-theory, GUTs and the Weak Scale, JHEP 09 (2009) 079 [arXiv:0809.1098] [INSPIRE].

[15] A. Font and L.E. Ibáñez, Yukawa Structure from U(1) Fluxes in F-theory Grand Unification, JHEP 02 (2009) 016 [arXiv:0811.2157] [INSPIRE].

[16] H. Hayashi, R. Tatar, Y. Toda, T. Watari and M. Yamazaki, New Aspects of Heterotic F-Theory Duality, Nucl. Phys. B 806 (2009) 224 [arXiv:0805.1057] [InSPIRE].

[17] B. Andreas and G. Curio, From Local to Global in F-theory Model Building, J. Geom. Phys. 60 (2010) 1089 [arXiv:0902.4143] [INSPIRE].

[18] J. Marsano, N. Saulina and S. Schäfer-Nameki, F-theory Compactifications for Supersymmetric GUTs, JHEP 08 (2009) 030 [arXiv: 0904.3932] [INSPIRE].

[19] A. Collinucci, New F-theory lifts. II. Permutation orientifolds and enhanced singularities, JHEP 04 (2010) 076 [arXiv:0906.0003] [INSPIRE].

[20] R. Blumenhagen, T.W. Grimm, B. Jurke and T. Weigand, F-theory uplifts and GUTs, JHEP 09 (2009) 053 [arXiv:0906.0013] [INSPIRE]. 
[21] J. Marsano, N. Saulina and S. Schäfer-Nameki, Monodromies, Fluxes and Compact Three-Generation F-theory GUTs, JHEP 08 (2009) 046 [arXiv:0906.4672] [INSPIRE].

[22] R. Blumenhagen, T.W. Grimm, B. Jurke and T. Weigand, Global F-theory GUTs, Nucl. Phys. B 829 (2010) 325 [arXiv: 0908.1784] [inSPIRE].

[23] J. Marsano, N. Saulina and S. Schäfer-Nameki, Compact F-theory GUTs with U(1) $P Q$, JHEP 04 (2010) 095 [arXiv: 0912.0272] [inSPIRE].

[24] T.W. Grimm, S. Krause and T. Weigand, F-Theory GUT Vacua on Compact Calabi-Yau Fourfolds, JHEP 07 (2010) 037 [arXiv: 0912.3524] [INSPIRE].

[25] M. Cvetič, I. Garcia-Etxebarria and J. Halverson, Global F-theory Models: Instantons and Gauge Dynamics, JHEP 01 (2011) 073 [arXiv: 1003.5337] [INSPIRE].

[26] C.-M. Chen, J. Knapp, M. Kreuzer and C. Mayrhofer, Global SO(10) F-theory GUTs, JHEP 10 (2010) 057 [arXiv: 1005.5735] [INSPIRE].

[27] C.-M. Chen and Y.-C. Chung, Flipped SU(5) GUTs from E8 Singularities in F-theory, JHEP 03 (2011) 049 [arXiv: 1005. 5728] [INSPIRE].

[28] T.W. Grimm and T. Weigand, On Abelian Gauge Symmetries and Proton Decay in Global F-theory GUTs, Phys. Rev. D 82 (2010) 086009 [arXiv: 1006. 0226] [inSPIRE].

[29] J. Knapp, M. Kreuzer, C. Mayrhofer and N.-O. Walliser, Toric Construction of Global F-theory GUTs, JHEP 03 (2011) 138 [arXiv:1101.4908] [INSPIRE].

[30] M.J. Dolan, J. Marsano, N. Saulina and S. Schäfer-Nameki, F-theory GUTs with U(1) Symmetries: Generalities and Survey, Phys. Rev. D 84 (2011) 066008 [arXiv:1102.0290] [INSPIRE].

[31] J. Marsano and S. Schäfer-Nameki, Yukawas, G-flux and Spectral Covers from Resolved Calabi-Yau's, JHEP 11 (2011) 098 [arXiv:1108.1794] [INSPIRE].

[32] T.W. Grimm, M. Kerstan, E. Palti and T. Weigand, Massive Abelian Gauge Symmetries and Fluxes in F-theory, JHEP 12 (2011) 004 [arXiv:1107.3842] [INSPIRE].

[33] D.R. Morrison and D.S. Park, F-Theory and the Mordell-Weil Group of Elliptically-Fibered Calabi-Yau Threefolds, JHEP 10 (2012) 128 [arXiv:1208.2695] [INSPIRE].

[34] C. Mayrhofer, E. Palti and T. Weigand, U(1) symmetries in F-theory GUTs with multiple sections, JHEP 03 (2013) 098 [arXiv:1211.6742] [INSPIRE].

[35] V. Braun, T.W. Grimm and J. Keitel, New Global F-theory GUTs with U(1) symmetries, JHEP 09 (2013) 154 [arXiv:1302.1854] [INSPIRE].

[36] J. Borchmann, C. Mayrhofer, E. Palti and T. Weigand, Elliptic fibrations for $\mathrm{SU}(5) \times \mathrm{U}(1) \times \mathrm{U}(1)$ F-theory vacua, Phys. Rev. D 88 (2013) 046005 [arXiv:1303.5054] [INSPIRE].

[37] M. Cvetič, D. Klevers and H. Piragua, F-Theory Compactifications with Multiple U(1)-Factors: Constructing Elliptic Fibrations with Rational Sections, JHEP 06 (2013) 067 [arXiv: 1303.6970] [INSPIRE].

[38] V. Braun, T.W. Grimm and J. Keitel, Geometric Engineering in Toric F-theory and GUTs with U(1) Gauge Factors, JHEP 12 (2013) 069 [arXiv:1306.0577] [INSPIRE].

[39] M. Cvetič, A. Grassi, D. Klevers and H. Piragua, Chiral Four-Dimensional F-theory Compactifications With SU(5) and Multiple U(1)-Factors, JHEP 04 (2014) 010 [arXiv:1306.3987] [INSPIRE]. 
[40] M. Cvetič, D. Klevers and H. Piragua, F-Theory Compactifications with Multiple U(1)-Factors: Addendum, JHEP 12 (2013) 056 [arXiv: 1307.6425] [INSPIRE].

[41] J. Borchmann, C. Mayrhofer, E. Palti and T. Weigand, SU(5) Tops with Multiple U(1)s in F-theory, Nucl. Phys. B 882 (2014) 1 [arXiv:1307.2902] [INSPIRE].

[42] M. Cvetič, D. Klevers, H. Piragua and P. Song, Elliptic fibrations with rank three Mordell-Weil group: F-theory with $\mathrm{U}(1) \times \mathrm{U}(1) \times \mathrm{U}(1)$ gauge symmetry, JHEP 03 (2014) 021 [arXiv: 1310.0463] [INSPIRE].

[43] I. Antoniadis and G.K. Leontaris, F-GUTs with Mordell-Weil U(1)'s, Phys. Lett. B 735 (2014) 226 [arXiv: 1404.6720] [INSPIRE].

[44] D. Klevers, D.K. Mayorga Peña, P.-K. Oehlmann, H. Piragua and J. Reuter, F-Theory on all Toric Hypersurface Fibrations and its Higgs Branches, JHEP 01 (2015) 142 [arXiv: 1408.4808] [INSPIRE].

[45] C. Lawrie, S. Schäfer-Nameki and J.-M. Wong, F-theory and All Things Rational: Surveying U(1) Symmetries with Rational Sections, JHEP 09 (2015) 144 [arXiv: 1504.05593] [INSPIRE].

[46] M. Cvetič, D. Klevers, H. Piragua and W. Taylor, General U(1) $\times$ U(1) F-theory compactifications and beyond: geometry of unHiggsings and novel matter structure, JHEP 11 (2015) 204 [arXiv: 1507.05954] [INSPIRE].

[47] M. Cvetič, A. Grassi, D. Klevers, M. Poretschkin and P. Song, Origin of Abelian Gauge Symmetries in Heterotic/F-theory Duality, JHEP 04 (2016) 041 [arXiv:1511.08208] [INSPIRE].

[48] Y. Kimura and S. Mizoguchi, Enhancements in F-theory models on moduli spaces of K3 surfaces with ADE rank 17, PTEP 2018 (2018) 043B05 [arXiv: 1712.08539] [INSPIRE].

[49] Y. Kimura, F-theory models on K3 surfaces with various Mordell-Weil ranks constructions that use quadratic base change of rational elliptic surfaces, JHEP 05 (2018) 048 [arXiv: 1802.05195] [INSPIRE].

[50] M. Cvetič, L. Lin, M. Liu and P.-K. Oehlmann, An F-theory Realization of the Chiral MSSM with $\mathbb{Z}_{2}$-Parity, JHEP 09 (2018) 089 [arXiv:1807.01320] [INSPIRE].

[51] K. Oguiso and T. Shioda, The Mordell-Weil lattice of a rational elliptic surface, Comment. Math. Univ. St. Pauli 40 (1991) 83.

[52] W. Lerche, P. Mayr and N.P. Warner, Noncritical strings, Del Pezzo singularities and Seiberg-Witten curves, Nucl. Phys. B 499 (1997) 125 [hep-th/9612085] [INSPIRE].

[53] O.J. Ganor, D.R. Morrison and N. Seiberg, Branes, Calabi-Yau spaces and toroidal compactification of the $N=1$ six-dimensional $E_{8}$ theory, Nucl. Phys. B 487 (1997) 93 [hep-th/9610251] [INSPIRE].

[54] J.A. Minahan, D. Nemeschansky and N.P. Warner, Investigating the BPS spectrum of noncritical E(n) strings, Nucl. Phys. B 508 (1997) 64 [hep-th/9705237] [INSPIRE].

[55] J.A. Minahan, D. Nemeschansky and N.P. Warner, Partition functions for BPS states of the noncritical $E_{8}$ string, Adv. Theor. Math. Phys. 1 (1998) 167 [hep-th/9707149] [INSPIRE].

[56] Y. Yamada and S.-K. Yang, Affine seven-brane backgrounds and five-dimensional $E_{N}$ theories on $S^{1}$, Nucl. Phys. B 566 (2000) 642 [hep-th/9907134] [InSPIRE]. 
[57] T. Eguchi and K. Sakai, Seiberg-Witten curve for the E string theory, JHEP 05 (2002) 058 [hep-th/0203025] [INSPIRE].

[58] T. Eguchi and K. Sakai, Seiberg-Witten curve for E string theory revisited, Adv. Theor. Math. Phys. 7 (2003) 419 [hep-th/0211213] [INSPIRE].

[59] S. Kamiya, Degenerations of the exceptional excellent families in the MWL algorithm (in Japanese), MSc Thesis Kobe University, Japan (2017).

[60] S. Mizoguchi and T. Tani, Looijenga's weighted projective space, Tate's algorithm and Mordell-Weil Lattice in F-theory and heterotic string theory, JHEP 11 (2016) 053 [arXiv: 1607.07280] [INSPIRE].

[61] P.S. Aspinwall and D.R. Morrison, Point-like instantons on K3 orbifolds, Nucl. Phys. B 503 (1997) 533 [hep-th/9705104] [INSPIRE].

[62] P.S. Aspinwall, $M$ theory versus F-theory pictures of the heterotic string, Adv. Theor. Math. Phys. 1 (1998) 127 [hep-th/9707014] [InSPIRE].

[63] P.S. Aspinwall, Aspects of the hypermultiplet moduli space in string duality, JHEP 04 (1998) 019 [hep-th/9802194] [INSPIRE].

[64] R. Friedman, J. Morgan and E. Witten, Vector bundles and F-theory, Commun. Math. Phys. 187 (1997) 679 [hep-th/9701162] [INSPIRE].

[65] M.B. Green, J.H. Schwarz and P.C. West, Anomaly Free Chiral Theories in Six-Dimensions, Nucl. Phys. B 254 (1985) 327 [inSPIRE].

[66] L.B. Anderson, J. Gray, N. Raghuram and W. Taylor, Matter in transition, JHEP 04 (2016) 080 [arXiv: 1512.05791] [INSPIRE].

[67] S. Mizoguchi, $E_{10}$ symmetry in one-dimensional supergravity, Nucl. Phys. B 528 (1998) 238 [hep-th/9703160] [INSPIRE].

[68] S. Mizoguchi and G. Schröder, On discrete U duality in M-theory, Class. Quant. Grav. 17 (2000) 835 [hep-th/9909150] [INSPIRE].

[69] M. Fukae, Y. Yamada and S.-K. Yang, Mordell-Weil lattice via string junctions, Nucl. Phys. B 572 (2000) 71 [hep-th/9909122] [INSPIRE].

[70] M. Bershadsky, K.A. Intriligator, S. Kachru, D.R. Morrison, V. Sadov and C. Vafa, Geometric singularities and enhanced gauge symmetries, Nucl. Phys. B 481 (1996) 215 [hep-th/9605200] [INSPIRE].

[71] S. Katz, D.R. Morrison, S. Schäfer-Nameki and J. Sully, Tate's algorithm and F-theory, JHEP 08 (2011) 094 [arXiv: 1106. 3854] [InSPIRE].

[72] D. Klevers and W. Taylor, Three-Index Symmetric Matter Representations of SU(2) in F-theory from Non-Tate Form Weierstrass Models, JHEP 06 (2016) 171 [arXiv: 1604.01030] [INSPIRE].

[73] T. Tani, Matter from string junction, Nucl. Phys. B 602 (2001) 434 [inSPIRE].

[74] S.B. Johnson and W. Taylor, Enhanced gauge symmetry in 6D F-theory models and tuned elliptic Calabi-Yau threefolds, Fortsch. Phys. 64 (2016) 581 [arXiv:1605.08052] [InSPIRE]. 